\title{
The E3 ligase TRIM1 ubiquitinates LRRK2 and controls its localization, degradation, and toxicity
}

Adrienne E. D. Stormo ${ }^{1}$, Molly FitzGibbon" ${ }^{18 \#, ~ F a r b o d ~ S h a v a r e b i ~}{ }^{18 \#, ~ E l i z a b e t h ~ M . ~}$

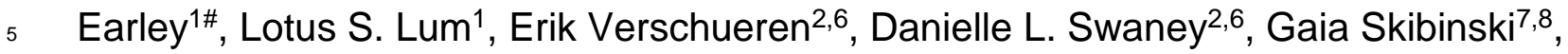
6 Abinaya Ravisankar ${ }^{7,8}$, Jeffrey van Haren ${ }^{3}$, Emily J. Davis ${ }^{1}$, Jeffrey R. Johnson ${ }^{2,6}$, John

7 Von Dollen ${ }^{2,6}$, Christian Mirescu ${ }^{9}$, Ciro laccarino ${ }^{10}$, William T. Dauer ${ }^{11-13}$, R. Jeremy 8 Nichols $^{14}$, Torsten Wittmann ${ }^{3}$, Timothy C. Cox ${ }^{15,16}$, Steve Finkbeiner $4,5,7,8$, Nevan J. 9 Krogan ${ }^{2,6,8}$, Scott A. Oakes ${ }^{1,17 *}$, and Annie Hiniker ${ }^{1,18^{*}}$.

Departments of Pathology ${ }^{1}$, Cellular and Molecular Pharmacology ${ }^{2}$, Cell and Tissue Biology ${ }^{3}$, Neurology 4 , and Physiology ${ }^{5}$, and the Quantitative Biosciences Institute ${ }^{6}$, University of California San Francisco, San Francisco, CA, 94143, U.S.A.; Taube/Koret Center for Neurodegenerative Disease Research ${ }^{7}$ and Center for Systems and Therapeutics 8 , J. David Gladstone Institutes, San Francisco, CA, 94158, U.S.A.; Neuroscience, Merck \& Co. Inc., Boston, MA USA ${ }^{9}$; Department of Biomedical Sciences ${ }^{10}$, University of Sassari, Sassari, Italy; Departments of Neurology and Neurotherapeutics ${ }^{11}$, Neuroscience ${ }^{12}$, and Peter O'Donnell Jr. Brain Institute ${ }^{13}$, University of Texas Southwestern Medical Center, Dallas, TX, 75380, U.S.A.; Department of Pathology ${ }^{14}$, Stanford University, Palo Alto, CA, 94304, U.S.A.; Department of Oral and Craniofacial Sciences ${ }^{15}$, School of Dentistry, and Department of Pediatrics ${ }^{16}$, School of Medicine, University of Missouri Kansas City, Kansas City, MO, 64108, U.S.A.; Department of Pathology ${ }^{17}$, University of Chicago, Chicago, IL, 60637, U.S.A.; Department of Pathology ${ }^{18}$, University of California, San Diego, CA, 92093, U.S.A.

\#Contributed equally

${ }^{*}$ Correspondence

soakes@bsd.uchicago.edu (Tel: 773-702-3797)

ahiniker@ucsd.edu (Tel: 858-246-5998) 


\begin{abstract}
:
Missense mutations in leucine-rich repeat kinase 2 (LRRK2) are the most common cause of familial Parkinson's Disease (PD); however, pathways regulating LRRK2 subcellular localization, function, and turnover are not fully defined. We performed quantitative mass spectrometry-based interactome studies to identify 48 novel LRRK2 interactors, including the microtubule-associated E3 ubiquitin ligase TRIM1

7 (Tripartite Motif Family 1). TRIM1 recruits LRRK2 to the microtubule cytoskeleton 8 for ubiquitination and proteasomal degradation by binding LRRK2822-982, a flexible $9 \quad$ interdomain region we designate the "Regulatory Loop" (RL). Phosphorylation of $10 \quad$ LRRK2 Ser910/935 within LRRK2 RL serves as a molecular switch controlling 11 LRRK2's association with cytoplasmic 14-3-3 versus microtubule-bound TRIM1. 12 Association with TRIM1 prevents upregulation of LRRK2 kinase activity by Rab29 and also rescues neurite outgrowth deficits caused by PD-driving mutant LRRK2 G2019S. Our data suggest that TRIM1 is a critical regulator of LRRK2, modulating its cytoskeletal recruitment, turnover, kinase activity, and cytotoxicity.
\end{abstract}




\section{Introduction:}

Leucine-rich repeat kinase 2 (LRRK2) mutations are the most common genetic cause of Parkinson's Disease (PD), a devastating neurodegenerative disorder affecting $1-2 \%$ of people over age $65 . .^{1,2}$ LRRK2 is a $290 \mathrm{kDa}$ polypeptide with multiple protein-protein interaction domains-including N-terminal armadillo, ankyrin, and LRR domains, and Cterminal WD40 repeats-that flank enzymatically active Roc GTPase (Ras of complex proteins), COR, and kinase domains (Figure 1a). Several point mutations in the catalytic core of LRRK2 cause autosomal dominant PD with incomplete penetrance (referred to herein as "LRRK2-PD"), while others mutations in the protein increase risk for sporadic PD. 3 , 4 The most common LRRK2-PD mutation, LRRK2 G2019S, falls in the kinase domain, as does the I2020T mutation. Several other PD-driving mutations, including $\mathrm{R} 1441 \mathrm{G} / \mathrm{C} / \mathrm{H}$ and $\mathrm{Y} 1699 \mathrm{C}$, are located in the Roc and COR domains and promote GTP binding. ${ }^{5,6} \mathrm{~A}$ distinct set of LRRK2 mutations augments risk for Crohn's disease, leprosy, and tuberculosis. ${ }^{7-10}$ How LRRK2 mutations drive PD is poorly understood, but mounting evidence supports a toxic gain-of-function mechanism that may involve abnormally increased kinase activity, increased LRRK2 protein levels, and possible changes in interacting partners and subcellular localization. ${ }^{11-13}$

The specific pathways regulating cellular LRRK2 turnover are only beginning to be comprehensively evaluated; however, both the autophagic-lysosome and ubiquitinproteasome systems appear to be involved. LRRK2 has a complex relationship with autophagy: multiple studies implicate LRRK2 in regulating autophagy, ${ }^{14,15}$ and a portion of LRRK2 appears to be degraded by chaperone-mediated autophagy. ${ }^{16}$ Additionally, a large fraction of endogenous LRRK2 has been shown to be degraded via the ubiquitinproteasome system; ${ }^{17}$ however, thus far, only two proteins are reported to act as E3 ubiquitin ligases for LRRK2: (1) WD repeat and SOCS box containing 1 (WSB1), which ubiquitinates LRRK2 via atypical K27 and K29 linkages and causes LRRK2 aggregation but does not appear to promote proteasomal degradation; ${ }^{18}$ and (2) C-terminus of Hsc70interacting protein (CHIP), an HSP70 co-chaperone that interacts with many partially folded proteins. ${ }^{19,} 20$ In keeping with its preference for misfolded proteins, ${ }^{21} \mathrm{CHIP}$ appears to be particularly important for turnover of destabilized LRRK2 variants, such as the 
sporadic PD modest risk allele LRRK2 G2385R, and may not be as critical for other LRRK2-PD mutants. ${ }^{22}$

LRRK2 is present at low levels in most cells types, hindering definitive determination of its endogenous subcellular localization. Predominantly through the use of overexpression systems, LRRK2 has been found (1) associated with membrane structures, where it interacts with Rab GTPases; ${ }^{23}$ (2) present in the cytoplasm, where it binds the 14-3-3 family of adapter proteins; ${ }^{24}$ and (3) at the cytoskeleton, where it interacts with microtubules. ${ }^{25}, 26$ Strong evidence demonstrates that LRRK2 associates with membranes; important recent work identified 14 membrane-associated Rab proteins as kinase substrates of LRRK2, including Rab10 and Rab29. ${ }^{27}$ Rab29, which localizes to Golgi network membranes, was also shown to be a unique activator of LRRK2's kinase activity, at least in cellular overexpression systems, as measured by LRRK2 autophosphorylation at Ser1292 and phosphorylation of substrate Rabs. ${ }^{23},{ }^{28}$ Rab29 appears to preferentially activate Roc-COR domain LRRK2-PD mutants such as LRRK2 R1441G. ${ }^{23}$ Rab29's exact binding sites on LRRK2 have not been fully described; however, conserved Leu-rich motifs in the ankyrin domain appear critical for the interaction and the armadillo repeats may also be important. ${ }^{23,29}$

LRRK2 also clearly localizes to the cytoplasm, where it associates with 14-3-3 proteins, a family of seven highly homologous isoforms that function as adaptor proteins to regulate myriad signaling pathways. ${ }^{30}$ The structural features mediating LRRK2's interaction with 14-3-3 have been rigorously investigated and phosphorylation of LRRK2 serine residues Ser910 and Ser935 is required. ${ }^{31}$ LRRK2-PD mutants with decreased phosphorylation of Ser910 and Ser935 (predominantly mutations in the Roc-COR domain) show reduced affinity for 14-3-3 proteins. ${ }^{11,31,32}$ LRRK2's interaction with 14-3-3 appears necessary to maintain LRRK2 in the cytoplasm and may be one mechanism that prevents Rab29mediated LRRK2 kinase activation. In support of this model, abnormal LRRK2 function was recently implicated in idiopathic PD (i.e., PD negative for LRRK2 mutations): sensitive proximity ligation assays were used to demonstrate both increased kinase activity and decreased 14-3-3 binding of LRRK2 in substantia nigra neurons from patients 
with idiopathic PD compared to controls, strengthening the link between abnormalities in LRRK2 function and idiopathic PD. ${ }^{33}$

A growing body of evidence indicates that LRRK2 can associate with the microtubule cytoskeleton. Multiple groups have demonstrated that LRRK2 forms filamentous structures along microtubules, ${ }^{25,26,34}$ which increase in frequency with kinase inhibitor treatment or point mutations in either the Roc-COR or kinase domains. ${ }^{25,26,34}$ Very recently, the in situ cryo-electron tomography structure of the PD-mutant LRRK2 I2020T bound to microtubules was solved to $14 \AA$, showing the Roc-COR domain adjacent to the microtubule and the kinase domain exposed to the cytoplasm. ${ }^{35}$ In keeping with this structure, LRRK2 can directly interact with $\beta$-tubulin through its Roc domain, inhibiting axonal transport. ${ }^{36-38}$ A second recent work, which solved the structure of LRRK2 to 3.5 A using cryo-EM, suggests that LRRK2's interaction with microtubules is regulated by the conformation of its kinase domain and further that LRRK2 binding to microtubules can disrupt axonal transport. ${ }^{39}$ Axonal transport is restored by increasing microtubule acetylation, suggesting that the LRRK2-microtubule interaction is regulated and occurs only at specific subtypes of microtubules. ${ }^{37}$ However, additional upstream signals or binding partners regulating LRRK2 localization to microtubules have not yet been identified.

Here, we used a mass spectrometry (MS) interactome approach to find new LRRK2 binding partners and discovered the little-studied E3 ubiquitin ligase TRIM1. While 14-33 stabilizes LRRK2 in the cytoplasm and Rab29 augments LRRK2's kinase activity at membranes, TRIM1 recruits LRRK2 to the microtubule cytoskeleton, where it mediates LRRK2 ubiquitination and proteasomal degradation. We narrowed down the TRIM1 binding site to nine amino acids (911-920) within a $\sim 50$ amino acid flexible interdomain ("Regulatory Loop" or RL) region of LRRK2, LRRK2822-982. LRRK2 RL contains Ser 910 and Ser 935, and the phosphorylation status of these serine residues influences LRRK2's choice of binding partner (14-3-3 vs TRIM1). Finally, TRIM1 inhibits Rab29-mediated activation of LRRK2 kinase activity and rescues PD-mutant LRRK2-driven toxicity as measured by neurite outgrowth. Our studies suggest that LRRK2's RL is a critical 
structural element whose post-translational modification controls its binding partners, including TRIM1, to regulate LRRK2 localization, turnover, kinase function, and toxicity.

\section{Results:}

\section{TRIM1 is a novel LRRK2 interacting partner}

We postulated that critical LRRK2 partners may have been missed in previous interaction studies, ${ }^{36,38,40}$ some of which used for bait a truncated LRRK2 fragment, LRRK2970-2527, which lacks the $\mathrm{N}$-terminal third of the protein. ${ }^{41}$ We therefore aimed to systematically and quantitatively identify the interactome of full-length LRRK2 in human cells using an established affinity purification-MS (AP-MS) approach. ${ }^{42} \mathrm{~N}$-terminally FLAG-tagged fulllength LRRK2 or FLAG-alone control plasmids were transiently transfected into HEK293T cells (selected for our extensive library of baseline interactome data, allowing better exclusion of non-specific interactors); lysates were affinity purified; and the eluted material subjected to MS as in Jager et al..$^{42}$ Interacting partners were determined by label-free MS1 quantification using MSStats. ${ }^{43}$ High-confidence interaction partners were proteins with an intensity $>3$-fold increased over empty vector control ( $p$-value $<0.05$ ), which identified $>20$ previously reported LRRK2-interacting proteins, including all members of the 14-3-3 family of proteins, as well as 48 novel partners, which were categorized according to function (Figure $1 \mathrm{~b}$ and Table S1). The top hit was the putative E3 ubiquitin ligase, tripartite motif family 1 (TRIM1, also called MID2), which has never been described as playing a role in LRRK2 biology, though a prior proteomics study did identify TRIM1 as a possible LRRK2 interacting partner in HEK-293T cells. ${ }^{40}$

TRIM1 is a little-studied $78 \mathrm{kDa}$ protein whose coding sequence is located on the $\mathrm{X}$ chromosome within the PARK12 genomic locus. ${ }^{44}$ TRIM1 belongs to a 75 member superfamily of E3 ubiquitin ligases with a common tripartite motif consisting of a RING domain, two B-box-type zinc fingers, and a coiled-coil domain. ${ }^{45}$ TRIM1's tripartite motif is followed by a microtubule-targeting COS domain, a fibronectin type III domain, and a C-terminal domain (Figure 1c). ${ }^{46}$ While its cellular functions remain largely uncharacterized, TRIM1 missense mutations were recently reported in families with a rare 
form of X-linked mental retardation, indicating a critical role in normal brain function. ${ }^{47}$ When exogenously expressed, TRIM1 and LRRK2 readily associated in cells (Figure 1d). To verify the interaction between endogenous LRRK2 and TRIM1, we immunoprecipitated TRIM1 from HEK293T cells and immunoblotted for LRRK2, with and without LRRK2 protein levels knocked down by siRNA (Figure 1e). Because TRIM1 is expressed at low levels endogenously, it is not visible by immunoblotting of HEK-293T cell lysates (asterisk indicates a non-specific band in the lysate). However, when endogenous TRIM1 was immunoprecipitated from HEK-293T cells, it was detectable by immunoblot, as was LRRK2. There was notably less LRRK2 coimmunoprecipitated with TRIM1 in the presence of LRRK2 siRNA, confirming the specificity of the LRRK2 band, and demonstrating that LRRK2 and TRIM1 interact under physiologic cellular conditions.

\section{TRIM1 recruits LRRK2 to microtubules}

Using overexpression studies, multiple groups have identified a portion of LRRK2 at microtubules. ${ }^{25,} 36$ The fraction of overexpressed LRRK2 associated with microtubules is typically low, although it can be increased with certain LRRK2 point mutations and kinase inhibitors. ${ }^{26}, 37$ We used live cell confocal microscopy to examine the subcellular distribution of transfected full-length GFP-LRRK2 in human lung H1299 cells. In agreement with previous studies, most GFP-LRRK2 was cytoplasmic and only a small fraction of overexpressed GFP-LRRK2 co-localized with microtubules labeled with mCherry-tubulin (Figure 2a). We confirmed previous work demonstrating that TRIM1 strongly binds to microtubules (Figure 2b). ${ }^{46,48,49}$ Strikingly, co-expression of mCherryTRIM1 substantially increased GFP-LRRK2 co-localization with microtubules (Figure 2b and Movie S1). We observed that mCherry-TRIM1 recruited GFP-LRRK2 to microtubules in all cell lines examined, including human lung carcinoma (A549) cells (Figure S1a), human neuroblastoma (SK-N-SH) cells (Figure S1b), and human embryonic kidney (HEK-293T) cells (Figure S1c), as well as human breast carcinoma (MCF7), and human (SH-SY5Y) and mouse (N2a) neuroblastoma cells (not shown). The fraction of GFPLRRK2 on microtubules in the absence of exogenous mCherry-TRIM1 was variable but typically low; however, in rare cells, a substantial proportion of overexpressed GFPLRRK2 localized to microtubules in the absence of exogenous TRIM1 (Movie S2). 
Microtubule-localized GFP-LRRK2 showed a discontinuous appearance in the presence as well as the absence of TRIM1 (Movie S2), in keeping with observations from other groups. $^{26}$

Repeated attempts under multiple experimental conditions did not allow us to visualize the subcellular localization of endogenous LRRK2. Live cell imaging was performed using A549 cells with an N-terminal GFP-tag added to LRRK2 by CRISPR editing (gift of Dario Alessi, unpublished). Immunofluorescence using a variety of commercially available LRRK2 antibodies (MJFF C41-2, UDD3, N231) was performed on the GFP-LRRK2 A549 line as well as wild type versus CRISPR LRRK2 knockout A549 cells (gift of Dario Alessi), wild type versus TALEN LRRK2 knockout murine RAW 264.7 macrophages (from the MJFF foundation), and wild type compared to siRNA LRRK2 knockdown human melanoma Malme-3M cells. Under no experimental condition could we visualize a fluorescence signal specific to LRRK2. The inability to reproducibly visualize endogenous LRRK2 using these methods is in keeping with previous reports and highlights an important limitation in the field..$^{50}$

We next evaluated the specificity of the TRIM1-LRRK2 interaction in controlling LRRK2 microtubule localization. Of the $\sim 75$ members of the TRIM family, TRIM1 is most homologous to TRIM18 (76\% identical, 88\% similar, Figure S1d). Like TRIM1, TRIM18 binds microtubules; however, loss-of-function mutations in TRIM18 cause a syndrome of congenital midline defects (X-linked Opitz G/BBB syndrome), which has not been observed for TRIM1 mutations. ${ }^{48}$ Intriguingly, myc-TRIM18 did not robustly coimmunoprecipitate with LRRK2 (Figure S1e) and co-expression of mCherry-TRIM18 was insufficient to recruit LRRK2 to microtubules (Figure 2c). We also tested the ability of LRRK2 to bind TRIM9, which has the same domain organization as TRIM1 and TRIM18 (25\% identical, $39 \%$ similar) and has been linked to PD in one study. ${ }^{51}$ As with TRIM18, myc-TRIM9 failed to appreciably co-immunoprecipitate with LRRK2 (Figure S1f). Thus, the LRRK2-TRIM1 interaction appears highly specific.

\section{TRIM1 ubiquitinates LRRK2 to regulate its turnover via the proteasome}


Given that many TRIM family members work as ubiquitin E3-ligases and that TRIM1 contains a RING domain, we speculated that TRIM1 may function to ubiquitinate LRRK2. We utilized an established in vivo ubiquitination assay for LRRK2, which was previously used to demonstrate LRRK2 ubiquitination by CHIP (the single E3 ubiquitin ligase known to target LRRK2 for proteasomal degradation). ${ }^{19}$ We found that coexpression of TRIM1 resulted in robust LRRK2 ubiquitination (Figure 3a, lane 2) with CHIP serving as a positive control (Figure 3a, lane 4). Because the RING domain is required for ubiquitin E3 ligase function of most TRIM proteins, we examined a TRIM1 mutant lacking the RING domain (TRIM1 $\triangle R F$ ) for its ability to ubiquitinate LRRK2. LRRK2 ubiquitination was markedly diminished in the presence of TRIM1 $\Delta R F$ (Figure S2a, compare eluate lane 3 to lane 1), confirming the importance of the RING domain to ubiquitin ligase function. The ability of TRIM18 to bind and ubiquitinate LRRK2 was also strikingly decreased compared to TRIM1 in co-immunoprecipitation experiments, consistent with TRIM18's inability to localize LRRK2 to microtubules (Figure S2a, compare eluate lane 2 to lane 1).

Polyubiquitin linkages frequently serve to target proteins for proteasomal or autophagic degradation, though they may also signal other molecular functions. ${ }^{52}$ Co-expression of TRIM1 with LRRK2 decreased LRRK2 accumulation over time compared to coexpression of control vector (Figure S2b, quantified in S2c), suggesting TRIM1-mediated ubiquitination of LRRK2 at microtubules might target LRRK2 for degradation. To specifically measure changes in LRRK2 turnover in vivo, we created a doxycycline ("dox")-inducible GFP-LRRK2 flow cytometric assay quantifying LRRK2 turnover: GFPLRRK2 expression was induced to measurable but near physiologic levels ( $\sim 10$ fold higher than endogenous LRRK2 expression), doxycycline removed, and GFP fluorescence measured by flow cytometry (Figure 3b). We first verified that normalized median GFP fluorescence intensity of GFP-LRRK2 was indeed proportional to LRRK2 levels on immunoblot (Figure S2d, S2e). We next tested the effect of TRIM1 expression on GFP-LRRK2 levels. As predicted, TRIM1 increased LRRK2 turnover-and thereby decreased LRRK2 levels (Figure 3c, quantified in 3d). LRRK2 levels did not change in the presence of TRIM1 $\Delta R F$, demonstrating TRIM1's effect was E3-ubiquitin ligase- 
dependent (Figure 3d). TRIM1 had no effect on turnover of dox-induced GFP alone (Figure S2f).

To further delineate the mechanism of TRIM1-mediated LRRK2 degradation, ubiquitinspecific MS was performed on immunoprecipitated FLAG-LRRK2 co-expressed with HAubiquitin and either myc-TRIM1, myc-TRIM1 $\Delta$ RF, or control vector. With $60 \%$ sequence coverage, a single site of ubiquitination, LRRK2 K831 was found to be dependent on TRIM1's E3 ubiquitin ligase activity (Table S2, Figure S3a and S3b). However, mutagenesis of this single LRRK2 lysine into an arginine residue (LRRK2 K831R) did not eliminate LRRK2 ubiquitination by TRIM1 (Figure S3c), indicating that additional LRRK2 lysines may be ubiquitinated, but were not identified in this experiment. TRIM1-dependent K48, K63, and K11 polyubiquitin linkages were identified in the eluate, suggesting turnover by either the proteasome (K48, K11) or autophagy (K63) (Figure S3b). This result was complicated by the strong binding of TRIM1 to LRRK2, and multiple TRIM1 auto-ubiquitination sites, ${ }^{53,54}$ thus the polyubiquitin chains identified in the eluate were not definitively bound to LRRK2. Therefore, we tested TRIM1-mediated LRRK2 degradation in the presence of MG132 (proteasome inhibitor) and chloroquine (autophagy inhibitor). LRRK2 degradation was inhibited by MG132, but not by chloroquine (Figure 3e), indicating that TRIM1-mediated degradation of LRRK2 occurs via the proteasome and not through autophagy. Finally, we compared the effects of TRIM1 on LRRK2 levels to the effects of TRIM18 and CHIP in our dox-inducible GFP. LRRK2 line. Neither TRIM18 nor CHIP significantly decreased wild type GFP-LRRK2 steady-state levels in this assay (Figure S3d). Thus, TRIM1 serves as a microtubulelocalized E3 ligase that ubiquitinates LRRK2, causing its degradation via the proteasome.

\section{Knockdown of endogenous TRIM1 increases LRRK2 levels}

To test the effect of endogenous TRIM1 on GFP-LRRK2 levels, we utilized a robust CRISPRi/dCas9 system ${ }^{55}$ to knockdown TRIM1 mRNA in conjunction with our flow cytometric GFP-LRRK2 assay. We generated dox-inducible GFP-LRRK2 cell lines stably expressing dCas9-BFP-KRAB. TRIM1 was knocked down using lentiviral transduction of single guide RNA (sgRNA) sequences targeted to the TRIM1 5' UTR. TRIM1 sgRNA 
lowered TRIM1 mRNA levels to $20.0 \%+/-4.4 \%$ compared to control sgRNA (Figure S3e) with a resulting increase in GFP-LRRK2 protein levels of $38.3 \%+/-3.3 \%$ at 24 hours (Figure 3f). This increase in GFP-LRRK2 protein levels was significant throughout the length of the experiment (up to 44 hours post-dox withdrawal) (Figure S3f), and the relative amount of GFP-LRRK2 protein present in the cells with TRIM1 sgRNA increased over time (Figure S3g), indicating a persistent LRRK2 turnover deficit in these cells. Thus, knockdown of endogenous TRIM1 decreases turnover of GFP-LRRK2, consistent with an important role for TRIM1 in LRRK2 degradation.

To examine the effects of TRIM1 on endogenous LRRK2 levels, we utilized human melanoma Malme-3M cells, which express relatively high levels of both LRRK2 and TRIM1 mRNA (NCBI Geoprofiles ID\#86805339 and \#86784306) and in which endogenous LRRK2 is quantifiable by immunoblot in whole cell lysate. SiRNA against TRIM1 was used to knockdown TRIM1 mRNA levels to $33 \%+/-6 \%$ relative to siRNA scrambled control (Figure S3h). TRIM1 knockdown resulted in an almost 2-fold increase $(162 \%+/-13 \%)$ in endogenous LRRK2 levels at 48 hours (Figures $3 g$ and $3 \mathrm{~h}$ ). Thus, TRIM1 is a critical regulator of endogenous LRRK2 turnover.

Small molecule LRRK2 kinase inhibitors have been shown to cause LRRK2 ubiquitination and proteasomal degradation and to increase LRRK2's microtubule association; ${ }^{39,} 56$ however, the pathways driving these phenomena have not been determined. Because the effects of TRIM1 expression phenocopy the effects of LRRK2 kinase inhibitor treatment (i.e. both cause LRRK2 ubiquitination, degradation, and localization to microtubules), we hypothesized that TRIM1 could mediate LRRK2 degradation following kinase inhibition. To test this, we first measured LRRK2 levels following treatment with the LRRK2 kinase inhibitor MLi-2 using the dCas9/dox-inducible LRRK2 system described above. We confirmed that MLi-2 treatment at $100 \mathrm{nM}$ for 24 hours decreases LRRK2 levels in this system, consistent with what others have observed (Figure S3i, compare vehicle to LRRK2 inhibitor treatment in the presence of control sgRNA). ${ }^{17,56} \mathrm{We}$ next examined the effects of kinase inhibition in the presence and absence of TRIM1 knockdown. If TRIM1 mediates LRRK2 degradation following MLi-2 treatment, we would 
expect that TRIM1 knockdown would rescue LRRK2 levels in the presence of MLi-2. As we had previously shown (see Figures 3f, S3f, S3g), TRIM1 knockdown increased LRRK2 levels in the absence of kinase inhibitor. TRIM1 knockdown caused no rescue of LRRK2 levels in the presence of MLi-2 compared to cells with a control sgRNA (Figure S3i; vehicle-treated LRRK2 levels are normalized to one). In control cells, MLi-2 treatment led to a $52.2 \%+/-3 \%$ decrease in total LRRK2 levels compared to vehicle, and in cells with TRIM1 knocked down, MLi-2 caused a 54.8\% +/- 4.4\% decrease in LRRK2 compared to vehicle (Figure S3i). We thus conclude that while TRIM1 mediates basal LRRK2 turnover, it does not drive kinase inhibitor-mediated LRRK2 degradation.

\section{TRIM1 mediates LRRK2 turnover in neurons}

We next tested TRIM1's ability to drive LRRK2 turnover in primary cortical neurons using optical pulse labeling, a method that has been used to monitor turnover of several neurodegenerative proteins, including huntingtin ${ }^{57}, \alpha$-synuclein ${ }^{58}$ and TDP- $43 .{ }^{59}$ In this assay, we fused the photoswitchable protein mEos3.2 to LRRK2. Cells expressing mEos3.2-LRRK2 initially fluoresce green; however, upon illumination with a 405nm wavelength light, a population of green-mEos3.2-LRRK2 is irreversibly switched to red, creating a distinct pool of red-mEos3.2-LRRK2 that can be followed using automated longitudinal imaging (Figure 4). Embryonic day 20-21 rat primary cortical neurons were co-transfected with pGW1-GFP as a morphology marker, pGW1-mEos3.2-LRRK2 and either TRIM1 or a control plasmid, and photoswitched with a 5-8-s pulse of light at 405$\mathrm{nm}$ wavelength. Neurons were imaged every $6-8 \mathrm{~h}$ for red and green fluorescence using custom-based automated algorithms to capture images in an unbiased and highthroughput manner. Representative neurons in the presence and absence of TRIM1 are shown in Figure 4. In this neuronal system, as in the HEK-293T cell system, LRRK2 decay was significantly accelerated by almost two-fold in the presence of TRIM1 ( $\mathrm{t}_{1 / 2}$ LRRK2 $24.9 \mathrm{~h}$ in the absence of exogenous TRIM1, $\mathrm{t}_{1 / 2}$ LRRK2 $15.9 \mathrm{~h}$ in the presence of exogenous TRIM1, $p=0.025,113$ and 87 neurons per group respectively, 3 independent experiments).

\section{The interdomain region between LRRK's ankyrin and LRR domain binds TRIM1}


In order to better define the TRIM1/LRRK2 interaction, we performed a series of coimmunoprecipitation experiments using truncation mutants of both proteins (domain structure of LRRK2 truncation mutants illustrated in Figure S4). We found that LRRK2 interacts with the tandem B-box domain of TRIM1 (Figure 5a) with binding most dependent on TRIM1's B-box1 domain (Figure 5b). Notably, with the exception of the extreme C-terminus, TRIM1's B-box1 domain includes the portion of least homology to TRIM18 (Figure S1d, double red line), suggesting that variations in this region may account for the differential ability of these highly homologous TRIM family members to bind LRRK2.

LRRK2 constructs lacking the interdomain region between the ankyrin and LRR domains (amino acids 822-982) were markedly reduced in their ability to bind full-length TRIM1 (Figure 5c, asterisks denote constructs with strongly decreased binding). A truncated LRRK2822-982 mutant was sufficient for binding to TRIM1 (Figure 5c, far right lane) and the interaction was further narrowed down to LRRK2 amino acids 911-919 (Figure 5d). LRRK2 $222-982$ is also necessary and sufficient for TRIM1-mediated LRRK2 localization to microtubules (Figure 5e, S4a-j). We observed that GFP-LRRK2822-982, which lacks LRRK2's enzymatic and protein-protein interaction domains, smoothly coated microtubules compared to full-length LRRK2 (Figure 5e compared to Figure $2 b$ ). We hypothesize that full-length LRRK2 at the microtubule cytoskeleton may oligomerize through its Roc and COR domains, as has been previously described, ${ }^{60}$ resulting in the discontinuous coating observed for the full-length protein. Interestingly, the interdomain region that binds TRIM1 is absent from LRRK2's closest homologue, LRRK1. ${ }^{61}$ This region is already known to be critical in mediating binding of 14-3-3 proteins, LRRK2's best-understood interactors. ${ }^{11}$ It has also been shown to undergo significant phosphorylation in response to upstream kinases, suggesting it is a key LRRK2 regulatory region. ${ }^{31}$ In silico modelling of the secondary structure of LRRK2822-982 predicts it to be $>75 \%$ unstructured and $>75 \%$ solvent exposed (www.predictprotein.org), indicating it is expected to be an accessible loop region. From hereon, we designate LRRK2822-982 the LRRK2 Regulatory Loop (LRRK2 $\mathrm{RL}$ ) region (Figure 5f). 


\section{LRRK2 RL phosphorylation influences TRIM1 versus 14-3-3 binding} Interaction of 14-3-3 with LRRK2 has been studied in detail. ${ }^{30}$ This interaction depends on the phosphorylation state of multiple LRRK2 serine residues, with Ser910 and Ser935 phosphorylation absolutely required for the LRRK2-14-3-3 interaction. ${ }^{62}$ Since Ser910 and Ser935 are located within the LRRK2 RL, directly adjacent to the 9 amino acids required for TRIM1 binding, we postulated that TRIM1 and 14-3-3 might compete for LRRK2 binding. We therefore examined the ability of LRRK2 to co-immunoprecipitate 143-3 in the presence and absence of TRIM1. GFP-LRRK2 co-immunoprecipitated V5-143-3 theta in the absence of myc-TRIM1, as reported in the literature (Figure 6a). ${ }^{11}$ In the presence of both V5-14-3-3 and myc-TRIM1, GFP-LRRK2 robustly coimmunoprecipitated myc-TRIM1 but only bound 19\% as much V5-14-3-3 $(p<0.01)$ as it did in the absence of myc-TRIM1 (Figure 6a, quantified in Figure 6b), demonstrating that TRIM1 can disrupt LRRK2's binding to 14-3-3. A non-phosphorylatable GFP-LRRK2 Ser910Ala Ser935Ala double mutant (GFP-LRRK2-SA) did not bind V5-14-3-3, as has been demonstrated by others. ${ }^{11}$ In contrast, GFP-LRRK2-SA co-immunoprecipitated Myc-TRIM1 to the same extent as GFP-LRRK2-WT (Figure 6a, 6b). Myc-TRIM1 did not change the lack of binding of 14-3-3 to GFP-LRRK2-SA (Figure 6a, 6b). Thus, TRIM1 strongly immunoprecipitates with both LRRK2 WT and LRRK2-SA, while 14-3-3 selectively immunoprecipitates with LRRK2 WT.

We hypothesized that Ser910 and Ser935 phosphorylation might serve as a molecular switch controlling LRRK2 RL's predilection for binding partners. We therefore measured the phosphorylation state of LRRK2 bound to either TRIM1 or 14-3-3 using quantitative immunoblot and phospho-specific antibodies against either phospho-Ser910 or phosphoSer935 LRRK2. LRRK2 bound to 14-3-3 showed markedly increased phosphorylation at both Ser910 and Ser935 as compared to LRRK2 bound to TRIM1 (Figure 6c, compare red (phospho):green (total) signal of immunoprecipitated LRRK2). We quantified the ratio of phospho-LRRK2:total LRRK2 for 14-3-3-bound LRRK2 and TRIM1-bound LRRK2 (normalized to phospho-LRRK2:total-LRRK2 in the input lysate) (Figure 6d). Ser935 phosphorylation of TRIM1-bound LRRK2 was 27\% of 14-3-3-bound LRRK2 (phosphoS935 LRRK2:total LRRK2 ratio was 0.26 +/- 0.13 for TRIM1-bound LRRK2 and 0.94 +/- 
0.13 for 14-3-3-bound LRRK2, $p<0.01$, Figure 6d). Similarly, LRRK2 Ser910 phosphorylation of TRIM1-bound LRRK2 was $28 \%$ of 14-3-3-bound LRRK2 (ratio of phospho-S910 LRRK2: total LRRK2 was $0.21+$ - 0.02 for TRIM1-bound LRRK2 and 0.74 +/- 0.12 for 14-3-3-bound LRRK2, $p<0.01$, Figure 6d). Thus, phosphorylation of LRRK2's $R L$ region influences LRRK2's affinity for partner proteins, with TRIM1-bound LRRK2 less likely to be phosphorylated than 14-3-3-bound LRRK2.

To visualize the subcellular localization of LRRK2 in the presence of both 14-3-3 and TRIM1, we created H1299 cells stably expressing GFP-LRRK2 and EBFP-14-3-3 theta. In these cells, both GFP-LRRK2 and EBFP-14-3-3 showed a diffusely cytoplasmic localization in the absence of mCherry-TRIM1 (Figure S5a). Upon transient transfection with mCherry-TRIM1, GFP-LRRK2 associated with microtubules, while EBFP-14-3-3 remained diffusely cytoplasmic (Figure 6e). Together, these data suggest that TRIM1 competes with 14-3-3 to preferentially bind non-phosphorylated LRRK2 and localize it to microtubules.

\section{TRIM1 inhibits LRRK2 kinase activation by Rab29}

Rab29, which is found at Golgi network membranes, was recently identified as a strong activator of LRRK2 kinase function, at least in cell-based overexpression systems. ${ }^{23}, 28$ Rab29 increases LRRK2 autophosphorylation at Ser1292 and LRRK2 phosphorylation of substrate Rab proteins (Rab10 at Thr73 and Rab29 at Thr71). ${ }^{23}$ Rab29 interacts with the $\mathrm{N}$-terminal portion of LRRK2, with the C-terminal half of LRRK2's armadillo domain is critical for Rab29 interaction. ${ }^{29}$ Conserved Leu-rich motifs in LRRK2's ankyrin domainwhich is adjacent to LRRK2 RL-are also essential for LRRK2 activation by Rab29. ${ }^{23}$ To measure TRIM1's effect on Rab29-mediated LRRK2 activation, we induced GFP-LRRK2 or GFP-LRRK2 R1441G expression and co-expressed myc-TRIM1 and/or HA-Rab29 via transient transfection. In these experiments, we provided continuous dox-induction until the time of harvest to maintain equivalent LRRK2 levels in the presence and absence of TRIM1. We confirmed previous reports that overexpressed Rab29 increases kinase activity of LRRK2 WT and LRRK2 R1441G (in our hands 2-4 fold), as measured by autophosphorylation at Ser1292 (Figure 7a, quantified in Figures 7b). ${ }^{23}$ Expression of 
TRIM1 with LRRK2 alone had no effect on Ser1292 phosphorylation, but when TRIM1 was expressed with Rab29 and LRRK2, Ser1292 phosphorylation levels were restored to levels observed in the absence of Rab29 over-expression (Figure 7a, 7b). We also quantified Rab29 phosphorylation at Thr71 and Rab10 phosphorylation at Thr73 as measures of LRRK2 kinase activity beyond autophosphorylation. Co-expression of TRIM1 caused a significant decrease in Rab29 phosphorylation by LRRK2 (for LRRK2 WT, 44\% +/- 5\% Rab29 phosphorylation in the presence of TRIM1 compared to the absence of TRIM1, $\mathrm{p}<0.05$; for LRRK2 R1441G, 58\% +/- 7\% Rab29 phosphorylation in the presence of TRIM1 compared to the absence of TRIM1, $p<0.05$; Figure 7a, quantified in 7c). Similarly, co-expression of TRIM1 caused a significant decrease in Rab10 phosphorylation by LRRK2 (for LRRK2 WT, 70\% +/- 9\% Rab10 phosphorylation in the presence of TRIM1 compared to the absence of TRIM1, $p<0.05$; for LRRK2 R1441G, $71 \%+/-8 \%$ Rab10 phosphorylation in the presence of TRIM1 compared to the absence of TRIM1, p<0.05; Figure 7a, quantified in 7d). Together, these data show that TRIM1 inhibits Rab29's ability to activate LRRK2's kinase function.

To begin to dissect the mechanism by which TRIM1 inhibits Rab29-mediated LRRK2 kinase activation, we utilized the TRIM1 $\Delta R F$ construct, which lacks E3 ligase function while maintaining microtubule localization and LRRK2 binding. To construct a cytoplasmic variant of TRIM1 with retained E3 ligase function, we turned to previous work on the highly homologous TRIM18, which showed that mutating six amino acids in TRIM18's COS domain to alanine prevents TRIM18 from binding to microtubules and redirects it to the cytoplasm. ${ }^{46}$ The identical amino acids are present in TRIM1 and were mutated to alanine (FLQ328AAA LDY377AAA, Figure S1d). The resulting construct, which we call TRIM1 C (for cytoplasmic), retains E3 ligase activity and LRRK2 binding (Figure S5b) but is diffusely cytoplasmic (Figure S5c). We found that, identical to WT TRIM1, TRIM1 C inhibits Rab29-mediated LRRK2 activation, as measured by Rab29 T71 phosphorylation (Figure 7e and S5d, quantified in Figure 7f). TRIM1 $\Delta$ RF did not inhibit Rab29-mediated LRRK2 activation, suggesting that TRIM1's E3 ubiquitin ligase function is required for this function. Mutation of Lys831, the ubiquitinated LRRK2 residue identified by MS, to Arg (LRRK2 K831R) did not rescue TRIM1's inhibition of Rab29-mediated LRRK2 activation 
(Figure $7 \mathrm{~g}$, quantified in Figure $7 \mathrm{~h}$ ). The mechanism by which TRIM1 ubiquitination inhibits Rab29-mediated LRRK2 activation requires further investigation.

TRIM1 rescues the neurite outgrowth defect caused by LRRK2 G2019S Of the LRRK2 PD-driving point mutations, the most common is LRRK2 G2019S. Similar to wild type LRRK2, LRRK2 G2019S was drawn to microtubules (Figure 8a), ubiquitinated (Figure 8b), and degraded via the proteasome (Figure 8c) in a TRIM1-dependent manner. LRRK2 G2019S expression is known to cause deficiencies in neurite outgrowth, a microtubule-driven process thought to reflect neuronal health. ${ }^{63,64}$ To test the effect of TRIM1 on neurite outgrowth deficiencies caused by expression of LRRK2 G2019S, we used a previously published rat PC12 pheochromocytoma cell line harboring doxinducible LRRK2 G2019S. ${ }^{65}$ These cells were transfected with mCherry-TRIM1 or control vector, dox-induced, and treated with nerve growth factor (NGF) for 4 days to induce neurite outgrowth. The proportion of cells with neurites (defined as cellular process greater than cell body length) ${ }^{66}$ was quantified (Figure $8 d, 8 e$ ), as was the length of those neurites (Figure 8f). Expression of TRIM1 alone did not affect PC12 neurite outgrowth (49\% +/- 2\% neurite-bearing cells without TRIM1 expression; 46\% +/- 9\% neurite-bearing cells with TRIM1 expression, $p=0.65$ ) (Figures 8d, quantified in 8e). LRRK2 G2019S expression, however, significantly reduced neurite outgrowth (Figure $8 \mathrm{~d}$ and $8 \mathrm{e}$ ), a phenotype that was completely rescued by co-expression of TRIM1 $(29 \%+/-6 \%$ neuritebearing cells without TRIM1; 44\% +/- 6\% neurite-bearing cells with TRIM1; $p=0.03$ ). Additionally, in those cells bearing neurites, expression of TRIM1 alone did not affect length of neurites (Figure $8 \mathrm{f}$, normalized neurite length $102 \%+/-5 \%$ without TRIM1 expression; 109\% +/- 8\% with TRIM1 expression, $p=0.47$ ). LRRK2 G2019S expression reduced neurite length in the absence of TRIM1 expression. TRIM1 expression in the presence of LRRK2 G2019S expression fully rescued neurite length (normalized neurite length 83\% +/- 4\% with LRRK2 G2019S expression; 111\% +/- 8\% with LRRK2 G2019S expression and TRIM1 expression, $\mathrm{p}=0.003$ ). Thus, TRIM1 protects against LRRK2 G2019S-induced neurite outgrowth defects.

\section{Discussion:}


Here we performed mass spectrometry-based proteomics to discover 48 previously unknown LRRK2 interacting proteins. The top novel hit was TRIM1, a poorly studied E3 ubiquitin ligase that recruits LRRK2 to the microtubule cytoskeleton, causing LRRK2 polyubiquitination and proteasomal degradation. TRIM1 decreases levels of overexpressed LRRK2 in both cells lines and primary cortical neurons, and knockdown of endogenous TRIM1 increases steady-state levels of endogenous LRRK2. Until now, the ubiquitous protein CHIP was the only E3 ligase known to target LRRK2 to the proteasome. ${ }^{19-21}$ Interestingly, we found that TRIM1 robustly ubiquitinates wild type LRRK2 and drives its proteasomal degradation more strongly than CHIP in our doxinducible assay. TRIM1, a RING-domain E3 ligase, and CHIP, a Ubox E3 ligase, bind to different regions of the LRRK2 protein. While CHIP interacts with LRRK2's Roc domain, ${ }^{19}$ TRIM1 binds to a nine amino acid region within LRRK2 RL directly adjacent to critical regulatory phospho-sites. CHIP mediates turnover of many unstable proteins, ${ }^{21}$ and appears particularly important for degradation of destabilized LRRK2 variants, such as the sporadic-PD risk allele, LRRK2 G2385R. ${ }^{22}$ One possible hypothesis is that CHIP may be especially important in degradation of unstable, misfolded LRRK2, while TRIM1's role in LRRK2 degradation may be related to LRRK2's phosphorylation state and subcellular localization.

Our results support a model in which the $\mathrm{RL}$ is a critical protein interaction domain influencing LRRK2 (WT and PD) localization, turnover, kinase activity, and some measures of neurotoxicity (Figure 9). We find that TRIM1 and 14-3-3 both interact with LRRK2 RL. The LRRK2-14-3-3 interaction requires phosphorylation of LRRK2 Ser910/935, while the LRRK2-TRIM1 interaction can occur in the absence of Ser910/935 phosphorylation. 14-3-3 binding also stabilizes LRRK2 in the cytoplasm, ${ }^{62}$ whereas TRIM1 binding recruits LRRK2 to microtubules, leading to LRRK2 ubiquitination and degradation. These findings suggest that phosphorylation of Ser910/935 in the LRRK2 $\mathrm{RL}$ may serve as a molecular switch dictating LRRK2's interacting partners and subcellular localization. LRRK2's close homolog LRRK1, which has a very similar domain structure to $L R R K 2$, lacks the $R L$ region. $L R R K 1$ also contains no mutations linked to 
PD. ${ }^{67}$ It is tempting to speculate that the $\mathrm{RL}$ region may be an important regulator of PDrelevant LRRK2 functions.

TRIM1 inhibits Rab29-mediated activation of LRRK2's kinase function in an E3 ligase dependent manner. Like others, ${ }^{23}$ we find that Rab29 overexpression augments LRRK2 kinase function of both wild type and PD-mutant LRRK2. Rab29's activation of LRRK2 has been demonstrated to occur at the trans-Golgi network. ${ }^{23}$ Intriguingly, LRRK2 interaction with TRIM1 inhibits Rab29-mediated LRRK2 activation, as measured by phosphorylation of Rab29, Rab10, and LRRK2 Ser1292. TRIM1's inhibitory effect requires an intact E3 ligase domain but does not require microtubule binding, as cytoplasmic mutant TRIM1 C inhibits Rab29-dependent LRRK2 activation but TRIM1 $\triangle R F$, lacking the E3 ligase domain, does not. These data suggest that ubiquitination of LRRK2 may regulate LRRK2 kinase function. We attempted to identify site(s) of LRRK2 ubiquitination by TRIM1 and identified a single TRIM1-dependent LRRK2 site, LRRK2 K831, with $60 \%$ sequence coverage by mass spectrometry. However, a LRRK2 K831R mutation neither eliminated LRRK2 ubiquitination by TRIM1 nor restored Rab29-mediated LRRK2 kinase activation in the presence of TRIM1. Because LRRK2 is such a large (290 kDa) protein, 60\% coverage identified 92 of 176 Lys residues but left the ubiquitination state of 84 Lys residues unknown. Additionally, we have not ruled out the possibility that TRIM1 ubiquitinates a non-LRRK2 target to regulate LRRK2 kinase activity. Thus, TRIM1 ubiquitination sites on LRRK2 remain to be fully delineated as does the mechanism by which TRIM1 inhibits Rab29's activation of LRRK2 kinase.

This work uncovers the microtubule cytoskeleton as a site of LRRK2 turnover, a new role for the cytoskeleton in PD. Multiple groups have reported an association of LRRK2 with microtubules, ${ }^{34-37}$ including recent work that solved the structure of the LRRK2microtubule interface to $14 \AA,^{35}$ and a second recent study that suggests that LRRK2's direct interaction with microtubules is regulated by the conformation of its kinase domain. ${ }^{39}$ However, the physiologic function of LRRK2's microtubule association has not been rigorously investigated and some authors have suggested that LRRK2-microtubule 
filaments represent concentration-dependent "aggresomes." 23 Our findings that TRIM1, but not the highly homologous TRIM18, binds LRRK2 in a 9 amino acid region to promote endogenous LRRK2 degradation supports a model in which microtubule association plays a physiologic role in LRRK2 biology. TRIM1 and TRIM18 are known to ubiquitinate other substrates at microtubules, targeting them for degradation. TRIM1 ubiquitinates astrin, a microtubule-associated protein involved in cell division, to decrease astrin levels and promote cell division. ${ }^{68}$ TRIM18 binds alpha4, a regulatory subunit of protein phosphatase 2a (PP2a), at microtubules, causing ubiquitination and degradation of PP2a ${ }^{69}$ However, this is the first report that microtubules serve as a site for LRRK2 ubiquitination leading to its degradation.

Interestingly, while mCherry-TRIM1 coated microtubules in a uniform and smooth distribution (Figure 2b, left panel, inset), GFP-LRRK2 formed more discontinuous and punctate structures, possibly suggestive of multi-protein complexes (Figure 2b, middle panel, inset). We predict that, in addition to causing LRRK2 turnover, the association of TRIM1 and LRRK2 on the microtubule cytoskeleton is likely to have additional functions that are yet to be delineated. Others have recently demonstrated that LRRK2's phosphorylation of Rab8 and Rab10 plays a critical role in ciliogenesis ${ }^{27,} 70$ and centrosomal cohesion. ${ }^{71}$ Cilia and centrosomes are microtubule-based structures, and Rab8 and Rab10-positive vesicles intimately associate with them. ${ }^{70}$ One possibility is that endogenous LRRK2 undergoes regulated trafficking between physically proximate Rabpositive membranes, microtubules, and the cytoplasm. Because LRRK2 contains numerous protein-protein interaction domains and has many features of a scaffolding protein, identification of other LRRK2 microtubule complex members may provide important insight into additional functions and regulation, as well as potential therapeutic targets.

TRIM1 falls within the PARK12 genomic locus on the X-chromosome. ${ }^{44}$ While it is tempting to speculate that TRIM1 mutations be linked to PD, PARK12 is a large locus containing $\sim 600$ genes and much additional work remains to determine if TRIM1 mutations are causative for PD. Regardless, TRIM1 may serve as a novel therapeutic 
target for PD. In vitro, TRIM1 causes ubiquitination and degradation of the most common PD-driving LRRK2 variant, LRRK2 G2019S, and ameliorates LRRK2 G2019S-mediated neurite outgrowth defects. The mechanism by which LRRK2 G2019S inhibits neurite outgrowth is unknown; however, increases in both LRRK2 kinase activity and protein levels have been linked to neurotoxicity in PD, ${ }^{33}$ and we observe a role for TRIM1 in the regulation of both. In future work, it will be important to further investigate the detailed mechanisms underlying TRIM1's inhibition of LRRK2 kinase activation. In keeping with a role for abnormal proteostasis in PD, ${ }^{72,73}$ LRRK2 disease-driving mutations influence LRRK2 turnover and steady-state levels: LRRK2 G2019S has a longer half-life and increased protein levels ${ }^{16}$ and LRRK2 Crohn's disease and leprosy risk alleles have a shorter half-life and decreased protein levels. ${ }^{9,} 74$ Thus, defining the pathways that regulate LRRK2 stability could lead to novel strategies to combat not just PD but also other diseases.

\section{Author Contributions:}

Conceptualization, A.H., G.S., N.J.K., S.A.O.; Methodology, A.H., A.E.D.S., E.M.E, E.V., D.L.S., G.S., J.V.H, T.P.M., J.R.J., J.V.D., C.M., T.W., R.J.N., T.C.C., S.F., E.J.S.; Investigation, A.H., A.E.D.S., E.M.E., L.L., D.L.S, A.R., E.D.; Writing - Original Draft, A.H., S.A.O., A.E.D.S.; Writing - Review \& Editing, G.S., E.V., D.L.S, T.W., R.J.N., T.C.C., S.F., N.J.K.; Funding Acquisition, A.H., T.W., T.C.C., R.J.N., C.I., S.F., N.J.K., S.A.O; Resources, C.I.; Supervision, A.H., T.W., T.C.C., R.J.N., S.F., S.A.O., N.J.K.

\section{Acknowledgements:}

We thank Dario Alessi for use of his CRISPR edited GFP-LRRK2 A549 cell line. This work was made possible with support from NIH NINDS 5K08NS090633 (A.H.), DoD W81XWH-18-1-0376 (A.H.), the American Federation for Aging Research (A.H.), the PFCC and DRC center grant NIG P30 DK063720, NIH U54 HG008105 (S.F.), RF1AG058476 (S.F), Merck and Co. (S.F), the Head Start Program of the Michael J Fox Foundation (S.F.), the Taube/Koret Center (S.F), Michael J. Fox Foundation LRRK2 
Challenge 2014 ID9550 (C.I.), Fondazione Banco di Sardegna grant number 2014.0489 (C.I.), Regione Sardegna grant number CRP-78083 (C.I), S10 RR026758 (T.W.), NIH R01EY027810 (S.A.O.), and NIH R01CA219815 (S.A.O.).

\section{CONFLICT OF INTEREST}

S.A.O. is a founder, equity holder, and consultant for OptiKIRA, LLC (Cleveland, OH) and a consultant for Kezar Life Sciences (South San Francisco, CA).

\section{CONTACT FOR REAGENT AND RESOURCE SHARING}

Further information and requests for resources and reagents should be directed to and will be fulfilled by the Lead Contact, Annie Hiniker (ahiniker@health.ucsd.edu).

\section{MATERIALS AND METHODS}

Cell lines and tissue culture:

All cell lines were grown at $37^{\circ} \mathrm{C}$ in a humidified atmosphere with $5 \% \mathrm{CO}_{2}$. Human HEK293T cells and human A549 cells were cultured in Dulbecco's modified Eagle's medium (DMEM) with 10\% fetal bovine serum (FBS). Doxycycline-inducible GFP-LRRK2 HEK$293 T$ cell lines ${ }^{56}$ were cultured in DMEM with $10 \%$ tetracycline-free FBS, $10 \mu \mathrm{g} / \mathrm{ml}$ blasticidin and $100 \mu \mathrm{g} / \mathrm{ml}$ hygromycin. Human H1299 cells were cultured in RPMI media with 10\% FBS, $25 \mathrm{mM}$ Hepes, and $2.0 \mathrm{~g} / \mathrm{L} \mathrm{NaHCO}_{3}$. Malme-3M cells were grown in RPMI-1640 with 10\% FBS and 2 mM (1x) L-alanyl-L-glutamine (GlutaMAX, ThermoFisher). SK-N-SH human neuroblastoma cells were cultured in Eagle's Minimum Essential Medium (EMEM). Rat primary cultures of cortical neurons were created from rat pup cortices at embryonic days 20-21 and cultured and differentiated as previously described ${ }^{58}$ in Neurobasal growth medium with 2mM GlutaMAX, Pen/Strep and B27 supplement (NB media). Doxycycline-inducible LRRK2 rat PC-12 cell lines ${ }^{65}$ were grown in DMEM supplemented with 10\% horse serum, 5\% tetracycline-free FBS, $2 \mathrm{mM}$

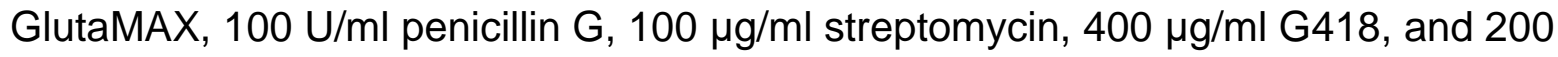
$\mu \mathrm{g} / \mathrm{ml}$ hygromycin and were differentiated under low-serum conditions the same media containing $1 \%$ horse serum without FBS and $100 \mathrm{ng} / \mathrm{ml} \mathrm{NGF.} \mathrm{PC12} \mathrm{cell} \mathrm{differentiation}$ media was replaced every 48 hours. 
Plasmids. Plasmids pcDNA5 frt/to expressing GFP-tagged human LRRK2, both fulllength and mutants and pCMV-C2-6myc or pCMV-C2-EGFP expressing WT human TRIM18 and TRIM1, both full-length and domain mutants have been previously described ${ }^{56,75}$ as has plasmid V5-14-3-3 theta ${ }^{26}$. Plasmid expressing mCherry-tubulin was a gift from Roger Tsien ${ }^{76}$, and plasmid pRK5-HA-ubiquitin WT was a gift from Ted Dawson (Addgene plasmid \#17608) ${ }^{77}$.

Full-length human LRRK2 with N-terminal myc and FLAG tags was cloned into pcDNA5 frt/to. In brief, pCMV-2myc-LRRK2, a gift from Mark Cookson (Addgene plasmid \#25361) ${ }^{78}$ was cloned into pcDNA5 frt/to by site-directed mutagenic removal of a single LRRK2 internal Hpal site (Quikchange, Stratagene), followed by Hpal/Eco53KI digest, ligation into EcoRV site, and return of Hpal site. A 2x FLAG tag was introduced upstream of the $2 x$ myc tag by quikchange (Quikchange, Stratagene). Addgene plasmid \#25361 was found to have the Arg50His variant not present in consensus Uniprot sequence (Q5S007) and site-directed mutagenesis was used to create Arg50.

Plasmid expressing GFP-LRRK2 $822-982$ was created by introducing a stop codon in GFPLRRK2822-2527 and plasmids expressing GFP-TRIM170-119 (denoted "linker" Figure 4b), GFP-TRIM1 70-177 (denoted "BB1", Figure 4b), and GFPTRIM170-235 (denoted "BB1,2", Figure $4 b$ ) were created by introducing stop codons into GFP-TRIM1 $1_{\triangle R F}$. Western blot was used to verify that there was no read-through of the stop codon.

Plasmid expressing mCherry-myc-TRIM1 was created by cloning myc-TRIM1 into pmCherry-C1 (Clontech). Plasmid expressing EBFP-14-3-3 was created using HiFi Cloning (NEB). A CHIP plasmid ${ }^{79}$ was a gift from Leonard Petrucelli and the CHIP ORF was cloned into pCMV-C2-6myc. Eos3.2-LRRK2 and mApple ORFs were synthesized and cloned into pGW1. All constructs were verified by DNA sequencing.

Antibodies. The following antibodies were used: mouse anti-LRRK2 (Neuromab, N241A/34), rabbit anti-LRRK2 (Abcam, C41-2), rabbit anti-phospho-Ser910 LRRK2 
(Abcam, UDD 1 15(3)), rabbit anti-phospho-Ser935 LRRK2 (Abcam, UDD2 10(12)), rabbit anti-FLAG (Sigma, F7425), mouse anti-myc (Sigma, clone 9E10, M4439), rabbit antimCherry (Abcam, ab167453), rabbit anti-TRIM1 (Sigma, M2448; Thermo PA5-28457), mouse anti-GFP (ThermoFisher, clone GF28R, MA5-15256), rabbit anti-HA (Cell Signaling, clone C29F4, 3724S), mouse anti-HA (Sigma, H7411), mouse anti-V5 (ThermoFisher, clone E 10/V4RR, MA5-15253) and mouse anti-actin (Sigma, A1978). For western blot, all primary antibodies were used at 1:1000 dilution except actin (1:1000$1: 5000$ ) and secondary antibodies (IRDye ${ }^{\circledR} 800 \mathrm{CW}$ or $680 \mathrm{RD}$ Goat anti-Mouse or antiRabbit IgG, LI-COR) were utilized at 1:10,000 dilution. For immunoprecipitation and coimmunoprecipitation, the following pre-conjugated agarose-resin systems were used according to manufacturer instructions: anti-FLAG M2 (Sigma, A2220); GFP-trap®_A or magnetic GFP-trap $\AA$ _MA, myc-trap $\AA_{-}$A or magnetic myc-trap ${ }^{\circledR} \_$MA or mCherry-trap ${ }^{\circledR} \_A$ (Chromotek); Pierce anti-HA (Thermo), and magnetic anti-V5 beads (MBL International).

Transfection and drug treatment. HEK-293T cells were transfected using Fugene 6 (Promega), Lipofectamine LTX, or Lipofectamine 2000 (Thermo-Fisher); H1299 cells were transfected with Lipofectamine 2000; and PC12 cells were transfected with Lipofectamine LTX or Lipofectamine 3000, all per manufacturer's instructions. LRRK2 expression was induced with $2 \mathrm{ng} / \mathrm{ml}$ to $1 \mu \mathrm{g} / \mathrm{ml}$ doxycycline. The proteasomal inhibitor MG132 was used at $2 \mu \mathrm{M}$ for 24 hours. Autophagy was inhibited with chloroquine at 25 $\mu \mathrm{M}$ for 24 hours. LRRK2 kinase inihibitor MLi-2 was used at $100 \mathrm{nM}$ for 24 hours.

For rat cortical neurons, at 3-4 days in vitro, neurons were transfected with plasmids and Lipofectamine 3000. Prior to adding the lipofectamine-DNA mix, cells were incubated in Neurobasal with 1x KY media (10 mM Kynurenic acid, 0.0025\% phenol red, $5 \mathrm{mM}$ HEPES and $100 \mathrm{mM} \mathrm{MgCl}$ ). Primary neurons were incubated in lipofectamine-DNA mix for 20-40 minutes, washed with Neurobasal, and cultured in NB medium.

\section{Immunoprecipitation, mass spectrometry, and data analysis of LRRK2}

interactome. $6 \mu \mathrm{g}$ of either pcDNA5 containing the above FLAG-LRRK2 construct or 
FLAG tag alone was transfected into HEK-293T cells using Fugene 6. Cells were harvested 48 hours after transfection using $10 \mathrm{mM}$ EDTA in D-PBS for $5 \mathrm{~min}$ at $25^{\circ} \mathrm{C}$, followed by 2 PBS washes. Cells were lysed in $1 \mathrm{ml}$ ice-cold immunoprecipitation buffer (50 mM Tris pH 7.5, $150 \mathrm{mM} \mathrm{NaCl}, 1 \mathrm{mM}$ EDTA, and 0.5\% NP-40) supplemented with cOmplete protease inhibitor (Roche) and Phosstop phosphatase inhibitor (Roche) and rotated end over end at $4^{\circ} \mathrm{C}$ for $30 \mathrm{~min}$ and debris pelleted at 3,000 rpm for $20 \mathrm{~min}$, and the resulting supernatant incubated with $30 \mu \mathrm{l}$ anti-FLAG M2 (Sigma, A2220) preconjugated agarose-resin for $2-3$ hours at $4^{\circ} \mathrm{C}$. FLAG affinity purification followed by mass spectrometry was carried out essentially as described in Jager et al. except that elution of bound proteins was done using $100 \mu \mathrm{g} / \mathrm{ml}$ FLAG peptide (Sigma, F3290). ${ }^{42} \mathrm{In}$ brief, following elution, $10 \mu \mathrm{l}$ of the IP eluate were reduced with $2.5 \mathrm{mM} \mathrm{DTT}$ at $60^{\circ} \mathrm{C}$ for 30 minutes followed by alkylation with $2.5 \mathrm{mM}$ iodoacetamide for 40 minutes at room temperature. $100 \mathrm{ng}$ sequencing grade modified trypsin (Promega) was then added to the sample and incubated overnight at $37^{\circ} \mathrm{C}$. Peptides were then desalted on ZipTip C18 pipette tips (Millipore), lyophilized to dryness, and resuspended in a final solution of $0.1 \%$ formic acid for injection into a Thermo Scientific LTQ Orbitrap XL Mass Spectrometer. For AP-MS experiments, raw data conversion and Protein Prospector search were also performed as described previously. ${ }^{42}$

Co-immunoprecipitation (co-IP). Cells were lysed in immunoprecipitation buffer (50 $\mathrm{mM}$ Tris $\mathrm{pH} 7.5,150 \mathrm{mM} \mathrm{NaCl}, 1 \mathrm{mM}$ EDTA, and 0.5\% NP-40) supplemented with cOmplete protease inhibitor (Roche) and Phosstop phosphatase inhibitor (Roche). Cells were lysed by pipetting up and down or by homogenization with pellet pestle on ice and cell lysates were rotated at $4^{\circ} \mathrm{C}$ for $30 \mathrm{~min}$ then debris pelleted at $4^{\circ} \mathrm{C}$ at $5,000 \mathrm{rpm}$ for 10 min. 20-40 $\mu$ l antibody-conjugated beads (GFP, myc, HA, or V5, listed above) were added and immunoprecipitations were performed at $4^{\circ} \mathrm{C}$ for 2-12 $\mathrm{h}$. Beads were washed $\geq 3 \mathrm{x}$ in wash buffer ( $50 \mathrm{mM}$ Tris $\mathrm{pH} 7.5,500 \mathrm{mM} \mathrm{NaCl}, 1 \mathrm{mM}$ EDTA) and bound proteins eluted by boiling in 40-80 $\mu \mathrm{l}$ x SDS loading dye. All imaging and quantification of immunoblots was performed using a LI-COR Odyssey® CLx imaging system. 
LRRK2 siRNA knockdown and co-immunoprecipitation with TRIM1. $800 \mathrm{nM}$ of either LRRK2 siRNA (Dharmacon Cat \#M-006323-02-0010) or control scrambled siRNA (\#D-0001206-13-05) was electroporated into HEK-293T cells according to the Lonza Kit $\checkmark$ protocol. After 48 hours growth at $37^{\circ} \mathrm{C}$, cells were harvested and lysed as above for co-IP. $50 \mu$ antibody-conjugated dynabeads (cat \#14311D), previously coupled to TRIM1 antibody (Thermo, PA5-28457) or IgG isotype control (BD Pharmingen, 554006), were incubated with lysates for 16 hours at $4^{\circ} \mathrm{C}$. Samples were washed, eluted, and immunoblotted as above.

Live cell microscopy. Spinning disk confocal live cell imaging was performed under environmentally controlled conditions, essentially as described ${ }^{80}$ except that the system was upgraded with a next generation scientific CCD camera (cMyo, 293 Photometrics) with 4.5 um pixels allowing optimal spatial sampling using a 60x NA 1.49 objective (CFI 294 APO TIRF; Nikon).

Flow Cytometry. GFP-LRRK2 levels were measured in doxycycline-inducible cell lines on a Fortessa flow cytometer (BD Biosciences). Cells were induced with 2-5 ng/ml doxycycline, and doxycycline was washed from cells 18-72 hours prior to analysis. GFP intensity was measured using a $488 \mathrm{~nm}$ laser for excitation, and a detector with $505-\mathrm{nm}$ long pass filter and a 525/50-nm band pass filter. Only live, single cells, as determined by forward and side scatter, were analyzed.

CRISPRi knockdown of TRIM1. Catalytically dead Cas9 (dCas9-BFP) was inserted randomly into doxycycline-inducible GFP-LRRK2 HEK-293T cells via lentiviral transduction. Cells were sorted for a BFP+ pure population on an Aria2SORP. BFP intensity was measured using a $405 \mathrm{~nm}$ laser for excitation, and a detector with a 450/50-nm band pass filter. The top four predicted guide RNAs for TRIM1 based on Horlbeck et al. ${ }^{81}$ were packaged with lentiviral vectors and added to cells, and then puromycin-selected $(0.75 \mu \mathrm{g} / \mathrm{mL})$ for 2 days before cells were plated and induced for included experiments. Knockdown was measured via real-time quantitative PCR with a non-targeting guide RNA used as a control. 
siRNA knockdown of TRIM1. 800 nM of either TRIM1 siRNA (Dharmacon Cat \#L006938-00-0005) or control scrambled siRNA (\#D-0001206-13-05) was electroporated into Malme-3m cells according to the Lonza Kit V protocol. After 24 hours incubation, cells were harvested and RNA was extracted following the NucleoSpin RNA Plus kit protocol (Macherey-Nagel) for qPCR. A TaqMan Gene Expression Assay probe against TRIM1/MID2 (Life Technologies Corporation) was used to confirm the knockdown of TRIM1 mRNA. After 48 hours, a parallel replicate of each knockdown was harvested, lysed, and immunoblotted for LRRK2 to measure the effect of TRIM1 knockdowns on LRRK2 protein levels. Protein levels were visualized using two antibodies against LRRK2, C41-2 and N241.

Identification of ubiquitinated LRRK2 lysines by MS. HEK-293T cells were seeded in a $15 \mathrm{~cm}$ cell culture dish and transfected with FLAG-LRRK2 and HA-ubiquitin plasmids in the presence of myc-TRIM1, myc- $\triangle R F$ TRIM1, or myc alone vector control. Sequential immunoprecipitation for FLAG and HA were performed on lysates as described above. Protein samples were subsequently reduced and alkylated, and digested with trypsin overnight at $37^{\circ} \mathrm{C}$. Peptides were then desalted on $\mathrm{C} 18$ ziptip columns, lyophilized to dryness, and resuspended in $0.1 \%$ formic acid for injection into an Orbitrap Fusion Lumos Tribrid Mass Spectrometer. Raw data was analyzed with MaxQuant to identify and quantify LRRK2 ubiquitination at K831, as well as ubiquitin chain abundance. ${ }^{82}$ Quantification across samples was normalized by LRRK2 protein abundance.

\section{LRRK2 turnover assay with robotic microscope imaging system. Primary rat} cortical neurons in 96-well plates were co-transfected with pGW1-GFP and pGW1mEos3.2-LRRK2, and either TRIM1 or control plasmid. To measure the degradation of LRRK2 neurons expressing pGW1-GFP, pGW1-Eos3.2-LRRK2, and either TRIM1 or empty vector were photoswitched with a 5-8 s pulse of light at $405-\mathrm{nm}$ wavelength 3036 hours after transfection. Prior to photoswitching neurons transfected with mEos3.2LRRK2 only fluoresce green with no detectable red fluorescence; however, upon photoswitching, a population of the green protein is irreversibly switched to emit red 
fluorescence. We then imaged the cells for red fluorescence (mEos3.2-LRRK2-red) every $4 \mathrm{~h}$ for the next 2 days.

Image analysis. Custom-based automated algorithms were used to capture images of neurons in each group in a high-throughput and unbiased manner. Live transfected neurons were selected for analysis based on pGW1-GFP fluorescence intensity and morphology. Neurons were only selected if they had extended processes at the start of the experiment. The abrupt loss of pGW1-GFP fluorescence was used to estimate the survival time of the neuron ${ }^{58,83}$. The Eos3.2-LRRK2-red fluorescence intensity was measured in each individual neuron at each time point that it was alive using a region of interest that corresponded to the cell soma. The co-transfected pGW1-GFP provided the morphology mask for the cell soma.

PC12 neurite outgrowth assay. Doxycycline-inducible LRRK2 G2019S PC12 cells were plated at 20,000 cells/well in 96-well plates and transfected with pmaxGFP at 30 ng/well (for analysis of cell morphology) and mCherry-TRIM1 or mCherry empty vector at $200 \mathrm{ng} /$ well. 24 hours after transfection, cells were moved to poly-d-lysine coated cover slips (Neuvitro) in the presence and absence of $1 \mu \mathrm{g} / \mathrm{ml}$ doxycycline. 48 hours after transfection, media was changed to PC12 differentiation media $+/-1 \mu \mathrm{g} / \mathrm{ml}$ doxycycline. Cells were then grown in differentiation media for 5 days with media changed every 48-60 hours. Cells were then fixed in 4\% paraformaldehyde-PBS for 20 min, washed $3 x$ with PBS, permeabilized in PBS with $10 \%$ goat serum, $0.4 \%$ Triton X$100,30 \mathrm{mg} / \mathrm{ml} \mathrm{BSA}$ and $10 \mathrm{mg} / \mathrm{ml}$ glycine for 1 hour, washed $3 x$ with PBS, and mounted on slides using Vectashield hardmount with DAPI. All steps from fixation to mounting were performed at $25 \mathrm{C}$. Cells were imaged at $40 \mathrm{X}$ using a Keyence BZ-X700 fluorescence microscope; all cells containing both red and green fluorescence were imaged. The presence/absence of neurites and length of neurites was assessed using ImageJ. Dead cells were excluded from further analysis.

\section{QUANTIFICATION AND STATISTICAL ANALYSIS}


Statistical analysis. Images shown represent typical results of independent experiments repeated at least three times. General statistical analysis was performed with excel software and indicated in figure legend. An unpaired, two-tailed Student's ttest was used to evaluate statistical significance. A p-value $<0.05$ was considered statistically significant.

Proteins were identified using Protein Prospector, and high-confidence proteininteractions were identified by label-free quantification of bait samples as compared to empty vector control using MSstats R-package ${ }^{43}$. Two independent experiments with two or three independent replicates of WT FLAG-LRRK2 compared to FLAG empty vector were included in the analysis. In the case of rare proteins in which peptides were seen in the presence of LRRK2 but none were identified in the empty vector control, such as TRIM1, a Mann-Whitney $U$ test was performed to identify proteins significantly increased in the LRRK2 sample.

Flow cytometry data was analyzed using FlowJo software (FlowJo LLC). Data represent the normalized median green fluorescence intensity and twice the standard error of the mean.

To measure the degradation of LRRK2 in the robotic microscope imaging system, RedLRRK2 fluorescence was measured longitudinally in each cell for at least 48h or until its death. The Red-LRRK2 intensity values from each cell were fitted to an exponential and used to derive a LRRK2 half-life value. Cells were excluded from the analysis if the Red-LRRK2 intensity values were lower than local background intensity. Cells without a monotonic decrease in Red-LRRK2 signal or with a half-life greater than three standard deviations from the mean were also excluded. The majority of these excluded cells were due to out of focus images. ANOVA analysis was used to compare significant differences between mean half-lives across the two groups.

Data Availability: The authors declare that the data supporting the findings of this study are available within the paper [and its supplementary information files]. 


\section{Main Figure Titles and Legends:}

Figure 1. TRIM1 is a new LRRK2 interacting partner. (A) Diagram of LRRK2 protein domains (ARM: armadillo repeat; ANK: ankyrin repeat; LRR: leucine-rich repeat; ROC: ras of complex proteins; COR: C-terminal of ROC domain). (B) Schematic of LRRK2 interactome in HEK-293T cells. LRRK2 interacting partners are classified radially according to function (aqua: new LRRK2 interacting partners, white: previously identified LRRK2 partners, size of circle indicates fold-change over empty vector control, circles without black outline had no peptides present in empty vector control, arrow indicates TRIM1). FLAG-LRRK2 was immunoprecipitated and interacting partners identified and quantified by MS. Data represent at least four total independent replicates from two experiments and are additionally shown in table S1. (C) Diagram of TRIM1 protein domains (FNIII: fibronectin III domain). (D) Co-immunoprecipitation of mycTRIM1 with FLAG-LRRK2 in HEK-293T cells. (E) Co-immunoprecipitation of endogenous LRRK2 with TRIM1 in HEK-293T cells. TRIM1 was immunoprecipitated from cells with or without LRRK2 knocked down by siRNA. Asterisk indicates a nonspecific co-reactive band in the whole cell lysate.

Figure 2. TRIM1 co-expression recruits LRRK2 to microtubules. (A) Live-cell confocal microscopy of GFP-LRRK2 and mCherry-tubulin or mCherry-TRIM1 transiently transfected into H1299 cells. In the presence of mCherry-tubulin, GFP-LRRK2 is diffusely cytoplasmic. From left to right: mCherry-tubulin, GFP-LRRK2, merged image. Inset shows higher magnification of region identified by box in merged image. (B) In the presence of mCherry-TRIM1, GFP-LRRK2 localizes to microtubules. From left to right: mCherry-TRIM1, GFP-LRRK2, merged image. Inset shows higher magnification of region identified by box in merged image. (C) In the presence of mcherry-TRIM18, GFPLRRK2 is diffusely cytoplasmic. From left to right: mCherry-TRIM18, GFP-LRRK2, merged image. Inset shows higher magnification of region identified by box in merged image.

Figure 3. TRIM1 ubiquitinates LRRK2 to regulate its turnover via the proteasome. 
(A) Co-immunoprecipitation and ubiquitination of FLAG-LRRK2 with myc-TRIM1 or mycCHIP in the presence of HA-ubiquitin in HEK-293T cells. (B) Schematic of flow cytometric assay using GFP fluorescence to measure GFP-LRRK2 turnover. Doxycycline-inducible GFP-LRRK2 HEK-293T cells are induced for 18 to 24 hours, transfected and doxycycline simultaneously withdrawn, and GFP fluorescence measured after 18-24 hours (additional validation of assay in Figure S2). (C) Representative histograms of GFP-LRRK2 fluorescence in the absence or presence of doxycycline followed by TRIM1 or empty vector transfection. (D) Quantification of GFPLRRK2 levels 24 hours after doxycycline withdrawal in the presence of empty vector (grey bar), TRIM1 (green bar), and ring-finger deleted $(\Delta R F)$ TRIM1 (purple bar). $(E)$ Quantification of GFP-LRRK2 levels in the presence of chloroquine at $25 \mu \mathrm{M}$ for 24 hours (CQ), MG132 at $2 \mu \mathrm{M}$ for 24 hours, or equivalent volume of DMSO vehicle. (F) Quantification of GFP-LRRK2 fluorescence with TRIM1 knocked down (red bar) compared to cells with non-targeting sgRNA (grey bar) 24 hours after dox withdrawal. (G) Immunoblot of endogenous LRRK2 in lysate of Malme-3M cells with scrambled siRNA (left lane) or endogenous TRIM1 knocked down by targeted siRNA (right lane). $(H)$ Quantification of $(G)$ showing mean value from six independent experiments with error bars showing standard error of the mean. All flow cytometry assays were performed in doxycycline-inducible GFP-LRRK2 HEK-293T cell lines described in Zhao et al. ${ }^{56}$ Bar graphs of flow cytometry assays represent normalized median green fluorescence intensity with error bars showing twice the standard error of the mean. All co-immunoprecipitation and flow cytometry assay results show a representative experiment, with the experiment repeated a minimum of 3 times. All histograms or bar charts of flow cytometry results represent at least 10,000 single cells per condition.

Figure 4. TRIM1 increases LRRK2 turnover in primary neurons. Representative primary cortical neurons co-transfected with mEos3.2-LRRK2 and GFP, and either TRIM1 or control plasmid were pulsed for 5-8 s with 405-nm light and imaged over the indicated time period. Prior to photoswitching (Pre-PS), mEos3.2-Red-LRRK2 is not detected. After photoswitching (Post-PS) mEos3.2-Red-LRRK2 is detected, nuclear 
excluded (arrow) and decays with time. LRRK2 decays faster in neurons transfected with TRIM1. Bar $10 \mu \mathrm{M}$.

\section{Figure 5. TRIM1 binds an N-terminal LRRK2 regulatory loop region via its $\mathrm{B}$-box} domain. (A) Co-immunoprecipitation of full-length myc-LRRK2 with GFP-TRIM1 domain constructs in HEK-293T cells ( $\triangle \mathrm{BB}$ : TRIM1 construct lacking both B-box domains; $\triangle \mathrm{CT}$ : TRIM1 lacking C-terminal domain, $\triangle R F$ : TRIM1 lacking ring-finger domain; $\triangle C C$ : TRIM1 lacking coiled coil domain; $\triangle \mathrm{FN} 3$ : TRIM1 lacking fibronectin III domain; details of constructs in ${ }^{75}$ ). (B) Co-immunoprecipitation of full-length myc-LRRK2 with GFP-TRIM1 B-box domain constructs in HEK-293T cells ( $\triangle$ RF denotes TRIM160-715; linker denotes TRIM160-117; BB1 denotes TRIM160-164; BB1,2 denotes TRIM160-212). (C) Coimmunoprecipitation of full-length myc-TRIM1 with GFP-LRRK2 domain constructs in HEK-293T cells. LRRK2822-892 is necessary and sufficient for interaction with TRIM1. (D) Co-immunoprecipitation of full-length myc-TRIM1 with GFP-LRRK2 with alanine mutagenesis within the RL. (E) Live-cell confocal microscopy of GFP-LRRK2 $822-982$ and mCherry-TRIM1 transiently transfected into H1299 cells. (F) Schematic of LRRK2TRIM1 domain interaction mediated by the LRRK2 Regulatory Loop (LRRK2822-982 (green)), labelled "RL" and TRIM1BBox1 (red). All co-immunoprecipitation experiments are a representative image of at least three independent experiments.

Figure 6. TRIM1 competes with 14-3-3 to bind LRRK2's regulatory loop and recruit LRRK2 to microtubules. (A) Co-immunoprecipitation of GFP-LRRK2 wild type (WT) and Ser910Ala Ser935Ala (SA) with V5-14-3-3 theta in the presence and absence of myc-TRIM1 in HEK-293T cells. (B) Quantification of (A) showing mean value from three independent experiments with error bars showing the standard error of the mean. (C) Co-immunoprecipitation of GFP-LRRK2 with either V5-14-3-3 theta or myc-TRIM1 in HEK-293T cells. Overlaid immunoblots in color show relative ratio of total to phosphoLRRK2 (total LRRK2 in green, antibody is NeuroMab clone N241A/34; phospho-LRRK2 is in red, antibodies are phospho-Ser910 (Abcam, UDD 1 15(3)) and phospho-Ser935 (Abcam, UDD 2 10(12)). (D) Quantification of (C) showing mean value from three independent experiments with error bars showing the standard error of the mean. $(E)$ 
Live-cell confocal microscopy of GFP-LRRK2 in the presence of mCherry-TRIM1 and EBPF2-14-3-3 theta. Inset shows GFP-LRRK2 and mCherry-TRIM1 at microtubules with EBFP-14-3-3 theta diffusely cytoplasmic. All live cell imaging and coimmunoprecipitation experiments are a representative image of at least three independent experiments.

Figure 7. TRIM1 decreases LRRK2's activation by Rab29. (A) Immunoblot of LRRK2 phosphorylation at Ser1292, Rab29 phosphorylation at Thr71, and Rab10 phosphorylation at Thr73 in the presence and absence of TRIM1 for wild type LRRK2 and LRRK2-PD mutant R1441G. (B) Quantification of LRRK2 autophosphorylation in (A). (C) Quantification of Rab29 Thr71 phosphorylation in (A). (D) Quantification of Rab10 Thr73 phosphorylation in (A). (E) Immunoblot of Rab29 phosphorylation with WT LRRK2 in the absence of TRIM1 or with overexpression of Myc-tagged wild type TRIM1, microtubule-nonbinding TRIM1 (TRIM1 C), or TRIM1 lacking E3-ligase function (TRIM1 $\Delta R F$ ). (F) Quantification of Rab29 phosphorylation in (E). (G) Immunoblot of Rab29 phosphorylation in the presence and absence of TRIM1 for wild type LRRK2 and LRRK2 K831R. (H) Quantification of Rab29 phosphorylation in (G). All immunoblots are representative images and quantification shows the mean value from three independent experiments with error bars showing the standard deviation.

\section{Figure 8. TRIM1 mediates proteasomal degradation of PD-mutant LRRK2 G2019S} to rescue its toxicity. (A) Live-cell confocal microscopy of GFP-LRRK2 G2019S and mcherry-TRIM1 transiently transfected into H1299 cells. (B) Co-immunoprecipitation and ubiquitination of GFP-LRRK2 G2019S with myc-TRIM1 in the presence of HAubiquitin in HEK-293T cells. (C) Flow cytometric assay on dox-inducible GFP-LRRK2 G2019S HEK-293T cells in the presence and absence of TRIM1 and the proteasome inhibitor MG132; bars show median green fluorescence intensity with error bars showing twice the standard error of the mean. (D) Representative dox-inducible LRRK2 G2019S PC-12 cells transfected with mCherry-TRIM1 or mCherry alone vector and GFP and differentiated with NGF for 5 days in the presence and absence of $1 \mu \mathrm{g} / \mathrm{ml}$ doxycycline. (E) Quantification of the fraction of neurite-bearing PC-12 cells in the 
presence and absence of LRRK2 G2019S and the presence and absence of TRIM1; bars show average of three independent experiments of 150-250 cells each, error bars show standard error of the mean. (F) Quantification of average neurite length on PC-12 cells with neurites in the presence and absence of LRRK2 G2019S and the presence and absence of TRIM1; bars show average of three independent experiments, error bars show standard error of the mean.

Figure 9. Model of the LRRK2 RL/TRIM1 interaction. Schematic shows the role of LRRK2 RL in controlling LRRK2 interaction with 14-3-3, Rab29, and Rab10 versus TRIM1, which alters LRRK2 localization, ubiquitination, proteasomal degradation, kinase activity, and toxicity.

Figure S1. Additional characterization of the LRRK2-TRIM1 interaction. Live-cell confocal microscopy of GFP-LRRK2 and mCherry-tubulin or mCherry-TRIM1 transiently transfected into (A) A549 cells, (B) SK-N-SH cells, or (C) HEK 293T cells. From left to right, each row shows mCherry-tubulin or mCherry-TRIM1, GFP-LRRK2, merged image. In all lines examined, in the presence of mCherry-tubulin, GFP-LRRK2 is diffusely cytoplasmic, but microtubule localized in the presence of mCherry-TRIM1. (D) Alignment of TRIM18 with TRIM1. Domains labeled above alignment. Red line designates region required for TRIM1 interaction with LRRK2. Double red line designates region of least homology in TRIM1 and TRIM18 dual B-box domain. DualAAA motifs below the sequence designate the mutated amino acids used to make cytoplasmic TRIM1 C variant. (E) Immunoprecipitation of GFP-LRRK2, which fails to coimmunoprecipitate with myc-TRIM18 in HEK293T cells. (F) Immunoprecipitation of GFP-LRRK2, which fails to co-immunoprecipitate with HA-TRIM9 in HEK-293T cells.

Figure S2. Evaluation of ubiquitination and degradation by TRIM18 and TRIM1 $\Delta R F$ and verification of flow cytometric system to measure LRRK2 turnover. (A) Coimmunoprecipitation and ubiquitination of FLAG-LRRK2 with myc-TRIM1, mycTRIM1 $\Delta$ RF, or myc-TRIM18 in the presence of HA-ubiquitin in HEK-293T cells. 
(B) Immunoblot of FLAG-LRRK2 co-transfected with myc-TRIM1 or empty vector control. Time indicates hours after transfection. (C) Quantification of panel (B) with LRRK2 levels normalized to actin. (D) Immunoblot showing LRRK2 levels relative to actin after withdrawal of doxycycline (doxycycline-induced for 18 hours). (E) Histograms of GFP fluorescence from samples immunoblotted in (D). (F) Immunoblot of doxinduced GFP expression co-transfected with myc-TRIM1 or empty vector control. Time indicates hours after transfection.

Figure S3. LRRK2 ubiquitination by TRIM1 and verification of TRIM1 knockdown. (A) Quantitative MS analysis of LRRK2 K831 ubiquitination in the presence of WT TRIM1, $\triangle$ RF TRIM1, or empty vector. (B) All ubiquitin linkages identified by MS analysis of ubiquitinated LRRK2 eluate in the presence of WT TRIM1, $\triangle R F$ TRIM1, or empty vector. (C) Co-immunoprecipitation and ubiquitination of GFP-LRRK2 WT or K831R with myc-TRIM1 in the presence of HA-ubiquitin in HEK-293T cells. (D) Quantification of GFP-LRRK2 levels in the presence of TRIM1, CHIP, or TRIM18. (E) Relative TRIM1 mRNA expression in dox-inducible GFP-LRRK2 HEK-293T cells with dCas9 and either non-targeting sgRNA (grey bar) or four pooled TRIM1-targeting sgRNAs (red bar). (F) Flow cytometric quantification of GFP-LRRK2 levels in TRIM1 knockdown and control lines at $0,4,24$, and 44 hours after dox withdrawal relative to 0 hours and (G) relative to control cells at all time points. $(\mathrm{H})$ Relative TRIM1 mRNA expression in Malme-3M cells with either scrambled siRNA (grey bar) or TRIM1-targeting siRNA (red bar). (I) Flow cytometric quantification of GFP-LRRK2 levels following LRRK2 inhibitor treatment (100 nM MLi-2 for 24 hours after dox withdrawal), normalized to LRRK2 levels with vehicle treatment. No difference was observed between cells with TRIM1 knocked down (red bars) compared to controls transduced with non-targeting sgRNA (grey bars). All histograms or bar charts of flow cytometry results represent at least 10,000 single cells per condition. Bars show median green fluorescence intensity with error bars showing twice the standard error of the mean.

Figure S4. TRIM1 binds LRRK2-RL to cause LRRK2 microtubule localization. Schematic of GFP-LRRK2 constructs (above) with corresponding live-cell microscopy in 
the presence of mCherry-TRIM1 in H1299 cells (below). Each panel shows only fluorescence at 488 (GFP) to illustrate the subcellular localization of each GFP-LRRK2 construct in the presence of mCherry-TRIM1, which is always localized to the microtubule network.

Figure S5. Characterization of LRRK2 co-expressed with 14-3-3 or cytoplasmic TRIM1. (A) Live-cell microscopy in the presence of mCherry-tubulin, GFP-LRRK2, and EBFP-14-3-3 in H1299 cells. (B) Co-immunoprecipitation and ubiquitination of GFPLRRK2 with myc-TRIM1 C in the presence of HA-ubiquitin in HEK-293T cells. (C) Livecell confocal microscopy of GFP-LRRK2 and mCherry-TRIM1 C transiently transfected into H1299 cells. From left to right, mCherry-TRIM1 C, GFP-LRRK2, merged image. (D) Immunoblot of Rab29 phosphorylation with LRRK2 R1441G in the absence of TRIM1 or with overexpression of Myc-tagged WT TRIM1, TRIM1 C, or TRIM1

Table S1, related to Figure 1. LRRK2 interacting partners.

Table S2, related to Figure 3. LRRK2 peptides and ubiquitination sites identified.

Movie S1, related to Figure 2. Time-lapse of GFP-LRRK2 localization in the presence of mCherry-TRIM1.

Movie S2, related to Figure 2. Time-lapse of rare cell with prominent GFP-LRRK2 microtubule localization in the presence of mCherry-tubulin (without mCherryTRIM1). 


\section{References:}

1. Ozelius, L.J. et al. LRRK2 G2019S as a cause of Parkinson's disease in Ashkenazi Jews. N Engl J Med 354, 424-425 (2006).

2. Klein, C. \& Westenberger, A. Genetics of Parkinson's disease. Cold Spring Harbor perspectives in medicine 2, a008888 (2012).

3. Kumari, U. \& Tan, E.K. LRRK2 in Parkinson's disease: genetic and clinical studies from patients. The FEBS journal 276, 6455-6463 (2009).

4. de Lau, L.M. \& Breteler, M.M. Epidemiology of Parkinson's disease. The Lancet. Neurology 5, 525-535 (2006).

5. Liao, J. et al. Parkinson disease-associated mutation R1441H in LRRK2 prolongs the "active state" of its GTPase domain. Proceedings of the National Academy of Sciences of the United States of America 111, 4055-4060 (2014).

6. Wu, C.X. et al. Parkinson's disease-associated mutations in the GTPase domain of LRRK2 impair its nucleotide-dependent conformational dynamics. The Journal of biological chemistry 294, 5907-5913 (2019).

7. Hartlova, A. et al. LRRK2 is a negative regulator of Mycobacterium tuberculosis phagosome maturation in macrophages. The EMBO journal 37 (2018).

8. Liu, Z. \& Lenardo, M.J. The role of LRRK2 in inflammatory bowel disease. Cell Res 22, 1092-1094 (2012).

9. Liu, Z. et al. The kinase LRRK2 is a regulator of the transcription factor NFAT that modulates the severity of inflammatory bowel disease. Nat Immunol 12, 1063-1070 (2011).

10. Zhang, F.R. et al. Genomewide association study of leprosy. N Engl J Med 361, 2609-2618 (2009).

11. Nichols, R.J. et al. 14-3-3 binding to LRRK2 is disrupted by multiple Parkinson's diseaseassociated mutations and regulates cytoplasmic localization. The Biochemical journal 430, 393404 (2010).

12. Steger, M. et al. Phosphoproteomics reveals that Parkinson's disease kinase LRRK2 regulates a subset of Rab GTPases. eLife 5 (2016).

13. Skibinski, G., Nakamura, K., Cookson, M.R. \& Finkbeiner, S. Mutant LRRK2 toxicity in neurons depends on LRRK2 levels and synuclein but not kinase activity or inclusion bodies. The Journal of neuroscience : the official journal of the Society for Neuroscience 34, 418-433 (2014).

14. Alegre-Abarrategui, J. et al. LRRK2 regulates autophagic activity and localizes to specific membrane microdomains in a novel human genomic reporter cellular model. Human molecular genetics 18, 4022-4034 (2009).

15. Plowey, E.D., Cherra, S.J., 3rd, Liu, Y.J. \& Chu, C.T. Role of autophagy in G2019S-LRRK2associated neurite shortening in differentiated SH-SY5Y cells. Journal of neurochemistry 105, 1048-1056 (2008).

16. Orenstein, S.J. et al. Interplay of LRRK2 with chaperone-mediated autophagy. Nat Neurosci 16, 394-406 (2013).

17. Lobbestael, E. et al. Pharmacological LRRK2 kinase inhibition induces LRRK2 protein destabilization and proteasomal degradation. Scientific reports 6, 33897 (2016).

18. Nucifora, F.C., Jr. et al. Ubiqutination via K27 and K29 chains signals aggregation and neuronal protection of LRRK2 by WSB1. Nature communications 7, 11792 (2016).

19. Ko, H.S. et al. CHIP regulates leucine-rich repeat kinase-2 ubiquitination, degradation, and toxicity. Proceedings of the National Academy of Sciences of the United States of America 106, 2897-2902 (2009). 
20. Ding, X. \& Goldberg, M.S. Regulation of LRRK2 stability by the E3 ubiquitin ligase CHIP. PloS one 4, e5949 (2009).

21. Edkins, A.L. CHIP: a co-chaperone for degradation by the proteasome. Subcell Biochem 78, 219242 (2015).

22. Rudenko, I.N. et al. The G2385R risk factor for Parkinson's disease enhances CHIP-dependent intracellular degradation of LRRK2. The Biochemical journal 474, 1547-1558 (2017).

23. Purlyte, E. et al. Rab29 activation of the Parkinson's disease-associated LRRK2 kinase. The EMBO journal 37, 1-18 (2018).

24. Rudenko, I.N. \& Cookson, M.R. 14-3-3 proteins are promising LRRK2 interactors. The Biochemical journal 430, e5-6 (2010).

25. Caesar, M. et al. Leucine-rich repeat kinase 2 functionally interacts with microtubules and kinase-dependently modulates cell migration. Neurobiology of disease 54, 280-288 (2013).

26. Kett, L.R. et al. LRRK2 Parkinson disease mutations enhance its microtubule association. Human molecular genetics 21, 890-899 (2012).

27. Steger, M. et al. Systematic proteomic analysis of LRRK2-mediated Rab GTPase phosphorylation establishes a connection to ciliogenesis. eLife 6 (2017).

28. Kalogeropulou, A., Freemantle, J., Lis, P., Vides, E., Polinski, N., Alessi, D. Endogenous Rab29 does not impact basal or nigericin and monensin stimulated LRRK2 pathway activity. BioRxiv https://doi.org/10.1101/2020.06.08.139675 (2020).

29. McGrath, E., Waschbusch, D., Baker, B.M. \& Khan, A.R. LRRK2 binds to the Rab32 subfamily in a GTP-dependent manner via its armadillo domain. Small GTPases, 1-14 (2019).

30. Cau, Y., Valensin, D., Mori, M., Draghi, S. \& Botta, M. Structure, Function, Involvement in Diseases and Targeting of 14-3-3 Proteins: An Update. Curr Med Chem 25, 5-21 (2018).

31. Muda, K. et al. Parkinson-related LRRK2 mutation R1441C/G/H impairs PKA phosphorylation of LRRK2 and disrupts its interaction with 14-3-3. Proceedings of the National Academy of Sciences of the United States of America 111, E34-43 (2014).

32. Li, X. et al. Phosphorylation-dependent 14-3-3 binding to LRRK2 is impaired by common mutations of familial Parkinson's disease. PloS one 6, e17153 (2011).

33. Di Maio, R. et al. LRRK2 activation in idiopathic Parkinson's disease. Science translational medicine 10 (2018).

34. Schmidt, S.H. et al. The dynamic switch mechanism that leads to activation of LRRK2 is embedded in the DFGpsi motif in the kinase domain. Proceedings of the National Academy of Sciences of the United States of America 116, 14979-14988 (2019).

35. Watanabe, R. et al. The In Situ Structure of Parkinson's Disease-Linked LRRK2. Cell (2020).

36. Law, B.M. et al. A direct interaction between leucine-rich repeat kinase 2 and specific betatubulin isoforms regulates tubulin acetylation. The Journal of biological chemistry 289, 895-908 (2014).

37. Godena, V.K. et al. Increasing microtubule acetylation rescues axonal transport and locomotor deficits caused by LRRK2 Roc-COR domain mutations. Nature communications 5, 5245 (2014).

38. Gandhi, P.N., Wang, X., Zhu, X., Chen, S.G. \& Wilson-Delfosse, A.L. The Roc domain of leucinerich repeat kinase 2 is sufficient for interaction with microtubules. $J$ Neurosci Res 86, 1711-1720 (2008).

39. Deniston, C.K. et al. Structure of LRRK2 in Parkinson's disease and model for microtubule interaction. Nature (2020).

40. Salasova, A. et al. A proteomic analysis of LRRK2 binding partners reveals interactions with multiple signaling components of the WNT/PCP pathway. Molecular neurodegeneration 12, 54 (2017). 
41. Beilina, A. et al. Unbiased screen for interactors of leucine-rich repeat kinase 2 supports a common pathway for sporadic and familial Parkinson disease. Proceedings of the National Academy of Sciences of the United States of America 111, 2626-2631 (2014).

42. Jager, S. et al. Global landscape of HIV-human protein complexes. Nature 481, 365-370 (2012).

43. Choi, M. et al. MSstats: an R package for statistical analysis of quantitative mass spectrometrybased proteomic experiments. Bioinformatics 30, 2524-2526 (2014).

44. Pankratz, N. et al. Genome-wide linkage analysis and evidence of gene-by-gene interactions in a sample of 362 multiplex Parkinson disease families. Human molecular genetics 12, 2599-2608 (2003).

45. Meroni, G. Genomics and evolution of the TRIM gene family. Advances in experimental medicine and biology 770, 1-9 (2012).

46. Short, K.M. \& Cox, T.C. Subclassification of the RBCC/TRIM superfamily reveals a novel motif necessary for microtubule binding. The Journal of biological chemistry 281, 8970-8980 (2006).

47. Geetha, T.S. et al. Targeted deep resequencing identifies MID2 mutation for X-linked intellectual disability with varied disease severity in a large kindred from India. Human mutation 35, 41-44 (2014).

48. Buchner, G. et al. MID2, a homologue of the Opitz syndrome gene MID1: similarities in subcellular localization and differences in expression during development. Human molecular genetics 8, 1397-1407 (1999).

49. Perry, J. et al. FXY2/MID2, a gene related to the X-linked Opitz syndrome gene FXY/MID1, maps to Xq22 and encodes a FNIII domain-containing protein that associates with microtubules. Genomics 62, 385-394 (1999).

50. Davies, P. et al. Comprehensive characterization and optimization of anti-LRRK2 (leucine-rich repeat kinase 2) monoclonal antibodies. The Biochemical journal 453, 101-113 (2013).

51. Tanji, K. et al. TRIM9, a novel brain-specific E3 ubiquitin ligase, is repressed in the brain of Parkinson's disease and dementia with Lewy bodies. Neurobiology of disease 38, 210-218 (2010).

52. Rajalingam, K. \& Dikic, I. SnapShot: Expanding the Ubiquitin Code. Cell 164, 1074-1074 e1071 (2016).

53. Chang, T.H., Yoshimi, R. \& Ozato, K. Tripartite Motif (TRIM) 12c, a Mouse Homolog of TRIM5, Is a Ubiquitin Ligase That Stimulates Type I IFN and NF-kappaB Pathways along with TNFRAssociated Factor 6. J Immunol 195, 5367-5379 (2015).

54. Yamauchi, K., Wada, K., Tanji, K., Tanaka, M. \& Kamitani, T. Ubiquitination of E3 ubiquitin ligase TRIM5 alpha and its potential role. The FEBS journal 275, 1540-1555 (2008).

55. Larson, M.H. et al. CRISPR interference (CRISPRi) for sequence-specific control of gene expression. Nat Protoc 8, 2180-2196 (2013).

56. Zhao, J., Molitor, T.P., Langston, J.W. \& Nichols, R.J. LRRK2 dephosphorylation increases its ubiquitination. The Biochemical journal 469, 107-120 (2015).

57. Tsvetkov, A.S. et al. Proteostasis of polyglutamine varies among neurons and predicts neurodegeneration. Nature chemical biology 9, 586-592 (2013).

58. Skibinski, G. et al. Nrf2 mitigates LRRK2- and alpha-synuclein-induced neurodegeneration by modulating proteostasis. Proceedings of the National Academy of Sciences of the United States of America 114, 1165-1170 (2017).

59. Barmada, S.J. et al. Autophagy induction enhances TDP43 turnover and survival in neuronal ALS models. Nature chemical biology (2014).

60. Deng, J. et al. Structure of the ROC domain from the Parkinson's disease-associated leucine-rich repeat kinase 2 reveals a dimeric GTPase. Proceedings of the National Academy of Sciences of the United States of America 105, 1499-1504 (2008). 
61. Sejwal, K. et al. Cryo-EM analysis of homodimeric full-length LRRK2 and LRRK1 protein complexes. Scientific reports 7, 8667 (2017).

62. Dzamko, N. et al. Inhibition of LRRK2 kinase activity leads to dephosphorylation of $\operatorname{Ser}(910) / \operatorname{Ser}(935)$, disruption of 14-3-3 binding and altered cytoplasmic localization. The Biochemical journal 430, 405-413 (2010).

63. MacLeod, D. et al. The familial Parkinsonism gene LRRK2 regulates neurite process morphology. Neuron 52, 587-593 (2006).

64. Sheng, Z. et al. Ser1292 autophosphorylation is an indicator of LRRK2 kinase activity and contributes to the cellular effects of PD mutations. Science translational medicine 4, 164ra161 (2012).

65. Migheli, R. et al. LRRK2 affects vesicle trafficking, neurotransmitter extracellular level and membrane receptor localization. PloS one 8, e77198 (2013).

66. Das, K.P., Freudenrich, T.M. \& Mundy, W.R. Assessment of PC12 cell differentiation and neurite growth: a comparison of morphological and neurochemical measures. Neurotoxicol Teratol 26, 397-406 (2004).

67. Reyniers, L. et al. Differential protein-protein interactions of LRRK1 and LRRK2 indicate roles in distinct cellular signaling pathways. Journal of neurochemistry 131, 239-250 (2014).

68. Gholkar, A.A. et al. The X-Linked-Intellectual-Disability-Associated Ubiquitin Ligase Mid2 Interacts with Astrin and Regulates Astrin Levels to Promote Cell Division. Cell Rep 14, 180-188 (2016).

69. Trockenbacher, A. et al. MID1, mutated in Opitz syndrome, encodes an ubiquitin ligase that targets phosphatase 2A for degradation. Nat Genet 29, 287-294 (2001).

70. Dhekne, H.S. et al. A pathway for Parkinson's Disease LRRK2 kinase to block primary cilia and Sonic hedgehog signaling in the brain. eLife 7 (2018).

71. Lara Ordonez, A.J. et al. RAB8, RAB10 and RILPL1 contribute to both LRRK2 kinase-mediated centrosomal cohesion and ciliogenesis deficits. Human molecular genetics 28, 3552-3568 (2019).

72. Balch, W.E., Morimoto, R.I., Dillin, A. \& Kelly, J.W. Adapting proteostasis for disease intervention. Science 319, 916-919 (2008).

73. Balchin, D., Hayer-Hartl, M. \& Hartl, F.U. In vivo aspects of protein folding and quality control. Science 353, aac4354 (2016).

74. Fava, V.M. et al. A Missense LRRK2 Variant Is a Risk Factor for Excessive Inflammatory Responses in Leprosy. PLoS Negl Trop Dis 10, e0004412 (2016).

75. Short, K.M., Hopwood, B., Yi, Z. \& Cox, T.C. MID1 and MID2 homo- and heterodimerise to tether the rapamycin-sensitive PP2A regulatory subunit, alpha 4, to microtubules: implications for the clinical variability of X-linked Opitz GBBB syndrome and other developmental disorders. BMC cell biology 3, 1 (2002).

76. Shaner, N.C. et al. Improved monomeric red, orange and yellow fluorescent proteins derived from Discosoma sp. red fluorescent protein. Nat Biotechnol 22, 1567-1572 (2004).

77. Lim, K.L. et al. Parkin mediates nonclassical, proteasomal-independent ubiquitination of synphilin-1: implications for Lewy body formation. The Journal of neuroscience : the official journal of the Society for Neuroscience 25, 2002-2009 (2005).

78. Greggio, E. et al. The Parkinson disease-associated leucine-rich repeat kinase 2 (LRRK2) is a dimer that undergoes intramolecular autophosphorylation. The Journal of biological chemistry 283, 16906-16914 (2008).

79. Petrucelli, L. et al. CHIP and Hsp70 regulate tau ubiquitination, degradation and aggregation. Human molecular genetics 13, 703-714 (2004).

80. Stehbens, S.J. et al. CLASPs link focal-adhesion-associated microtubule capture to localized exocytosis and adhesion site turnover. Nature cell biology 16, 561-573 (2014). 
81. Horlbeck, M.A. et al. Compact and highly active next-generation libraries for CRISPR-mediated gene repression and activation. eLife 5 (2016).

82. Brademan, D.R., Riley, N.M., Kwiecien, N.W. \& Coon, J.J. Interactive Peptide Spectral Annotator: A Versatile Web-based Tool for Proteomic Applications. Mol Cell Proteomics 18, S193-S201 (2019).

83. Arrasate, M., Mitra, S., Schweitzer, E.S., Segal, M.R. \& Finkbeiner, S. Inclusion body formation reduces levels of mutant huntingtin and the risk of neuronal death. Nature 431, 805-810 (2004). 
bioRxiy preprint doi: https://doi.org/10.1101/2020.10.21.336578; this version posted October 21, 2020. The copyright holder for this preprint

Figure 1 (which was not certified by peer review) is the author/funder. All rights reserved. No reuse allowed without permission.

\section{a $\quad$ LRRK2}

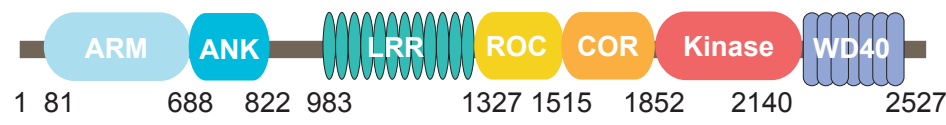

b

fold-change over empty vector

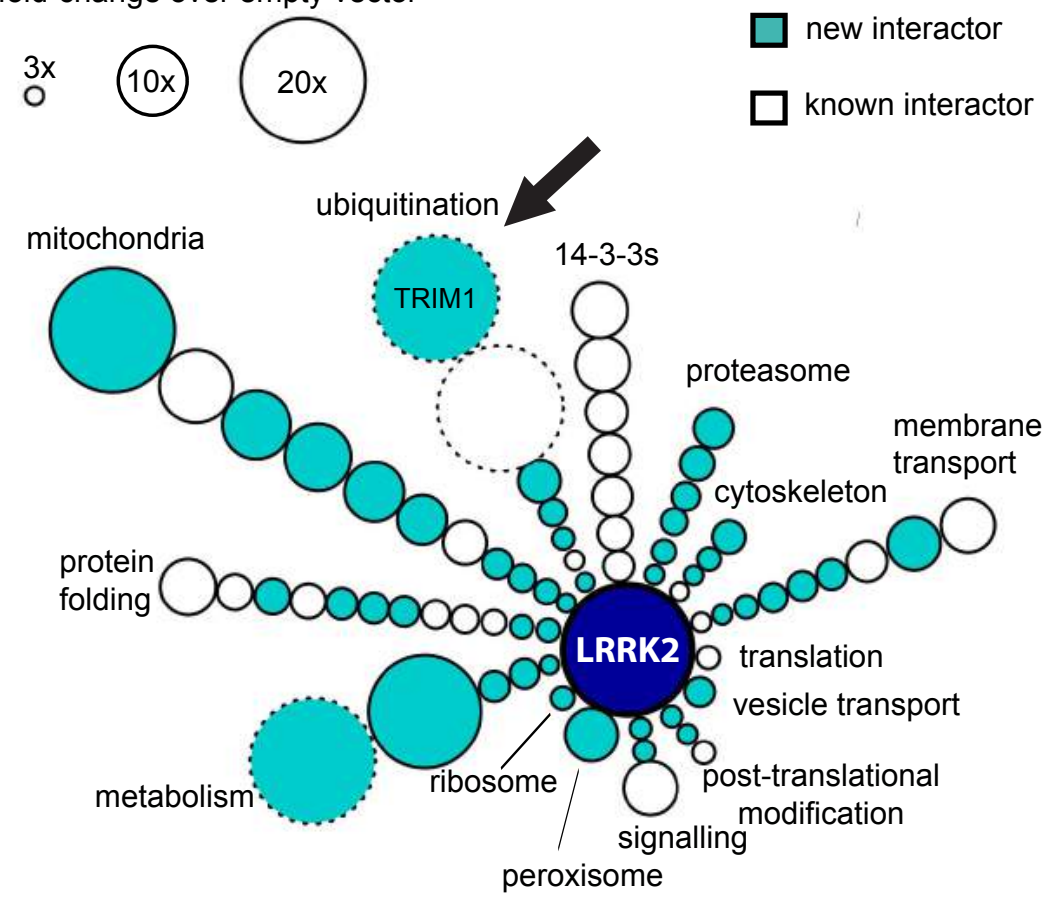

\section{TRIM1}

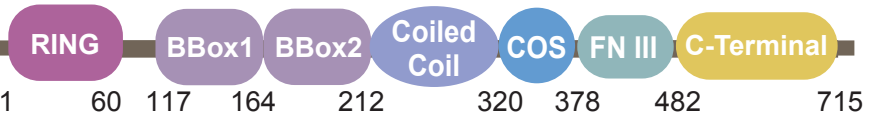

d

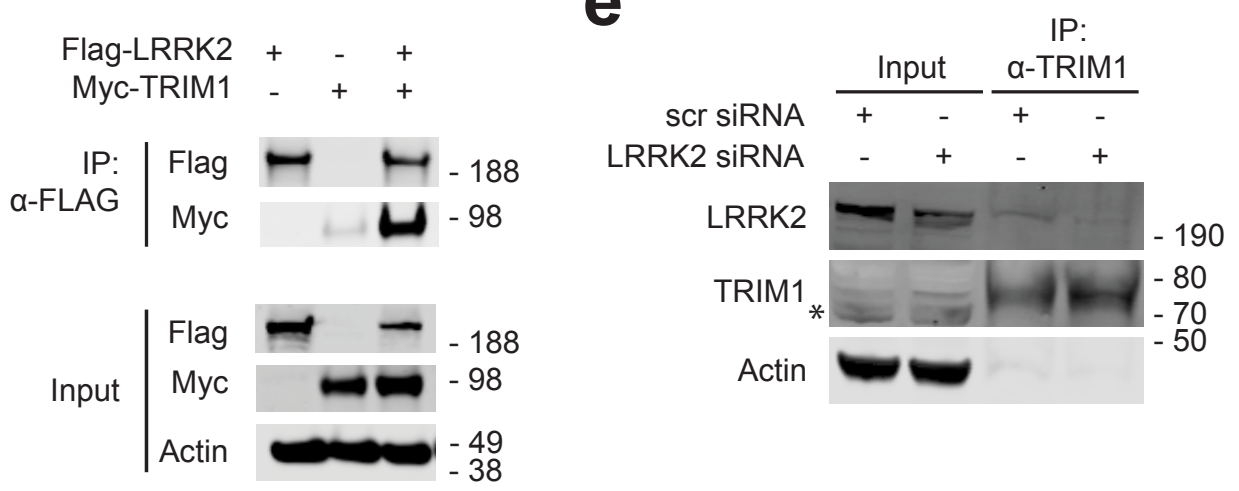


bioRxiv preprint doi: https://doi.org/10.1101/2020.10.21.336578; this version posted October 21, 2020. The copyright holder for this preprint

Figure 2 (which was not certified by peer review) is the author/funder. All rights reserved. No reuse allowed without permission.

a
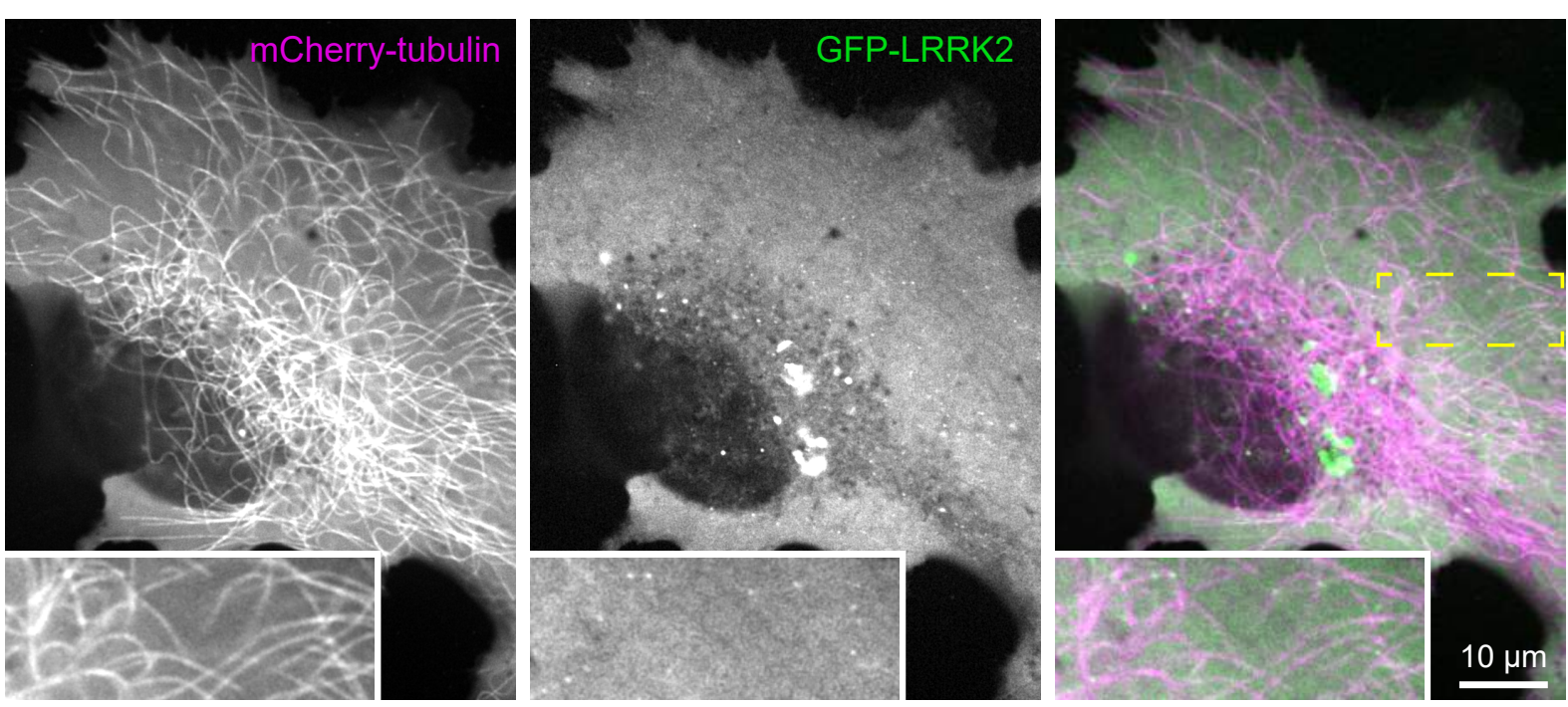

b
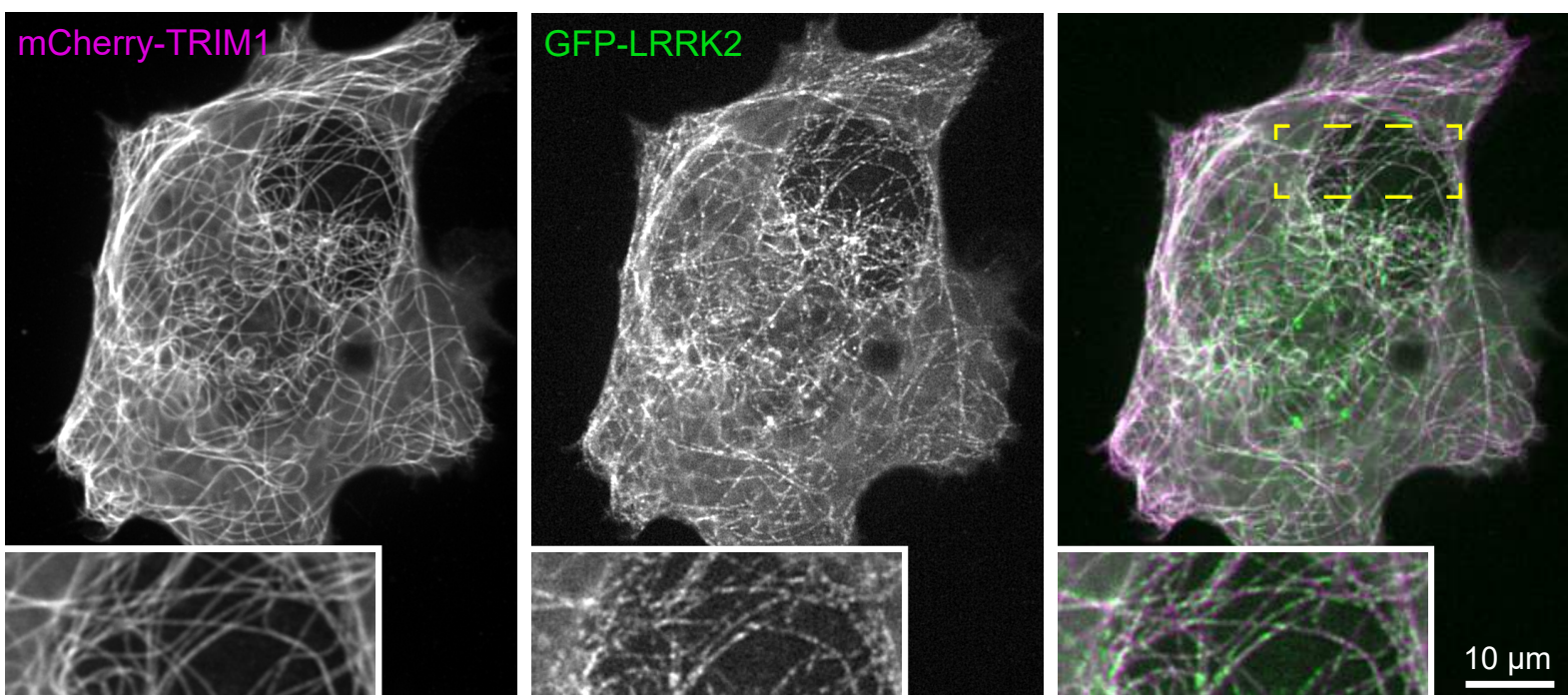

C
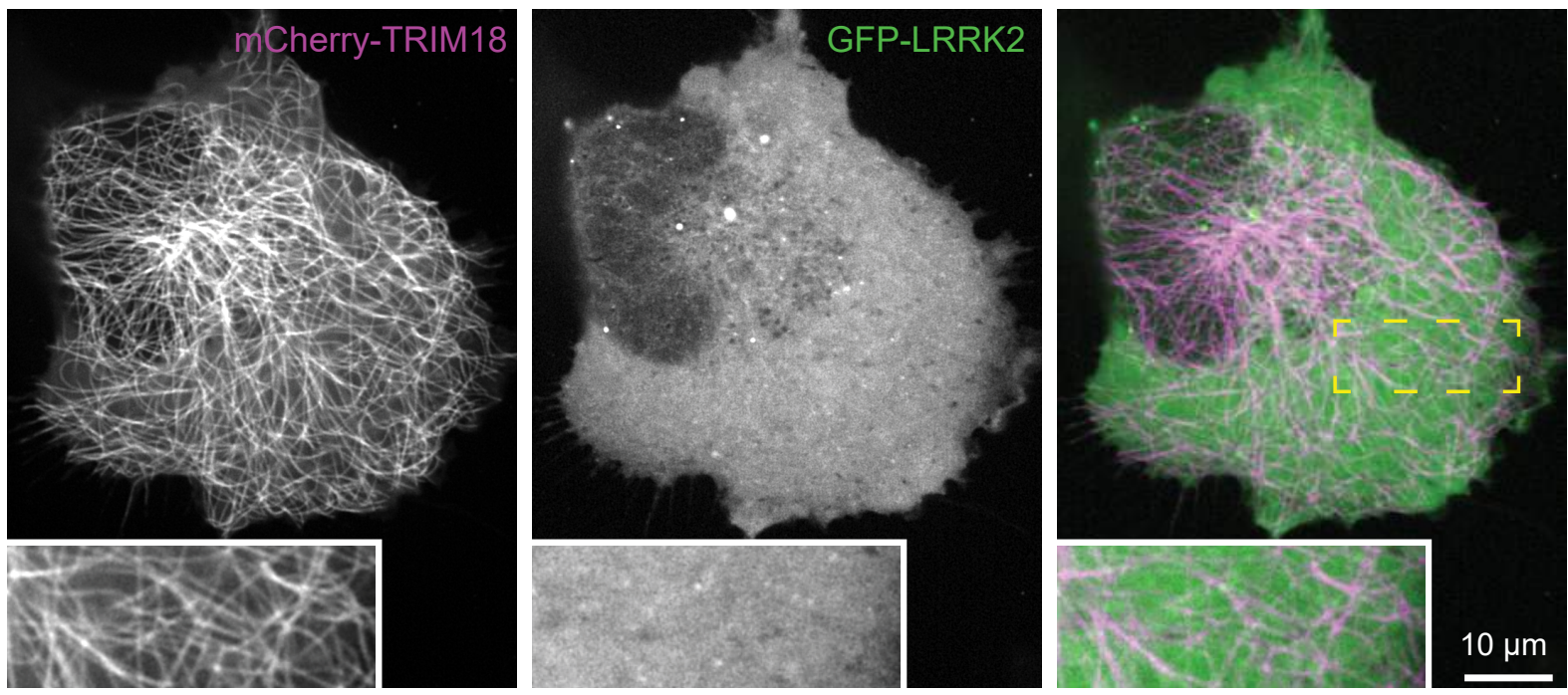
Fiqure 3 3iviv preprint doi: https://doi.org/10.1101/2020.10.21.336578; this version posted October 21, 2020. The copyright holder for this preprint Figure 3 (which was not certified by peer review) is the author/funder. All rights reserved. No reuse allowed without permission.

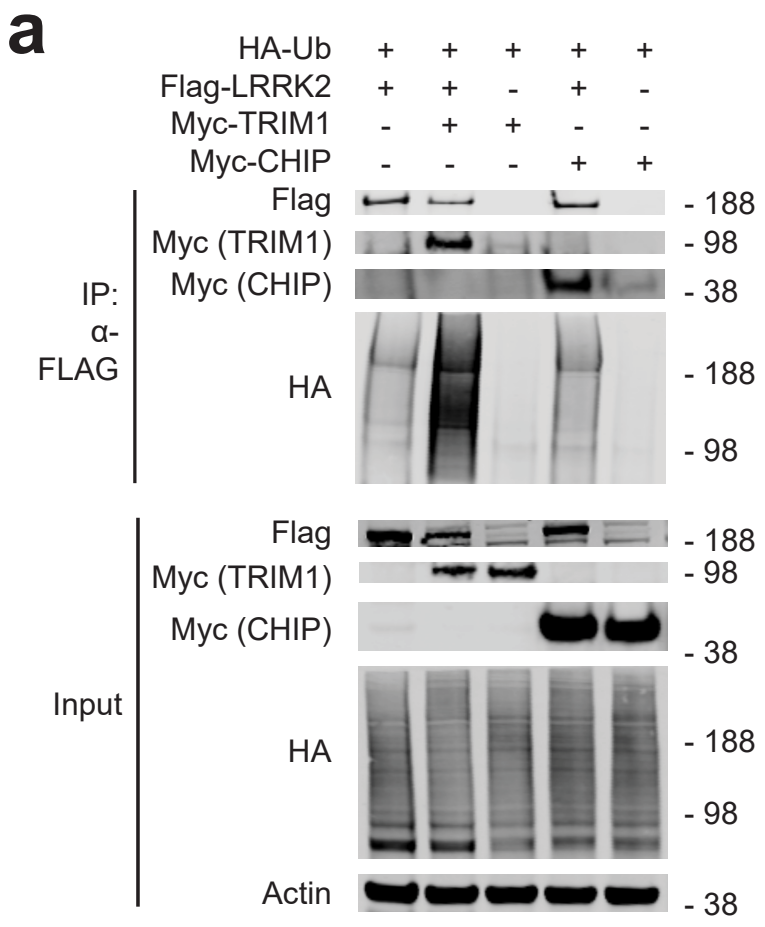

d

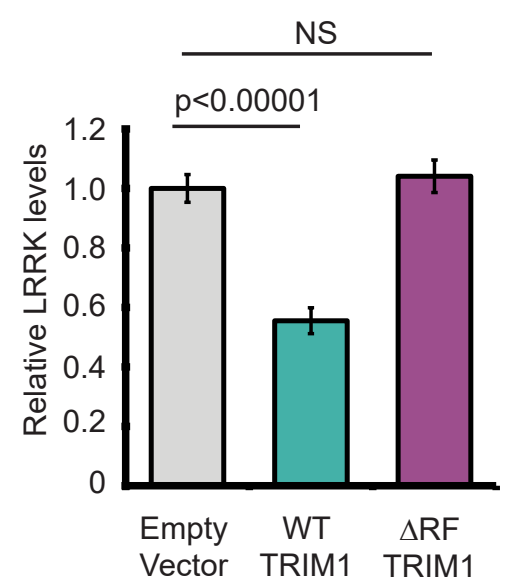

f

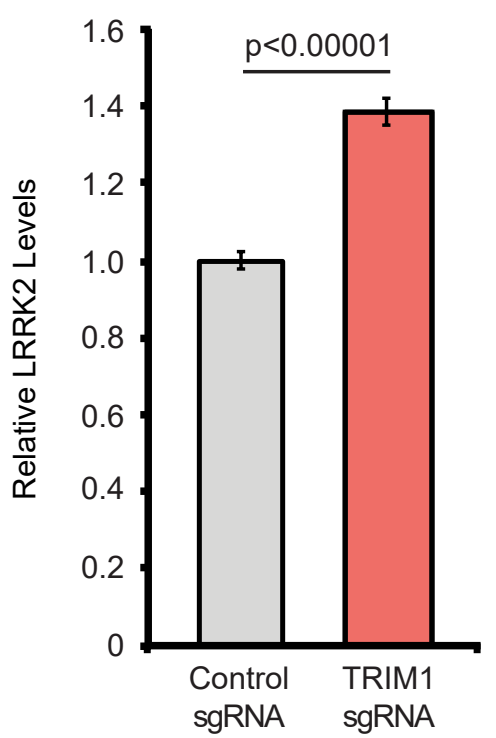

g b

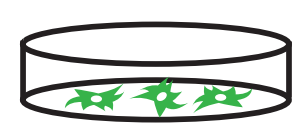

dox induction of tet-GFP-LRRK2
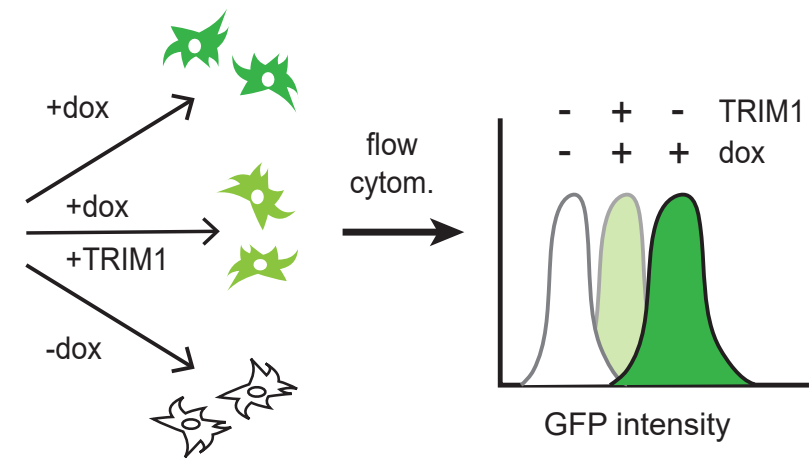

GFP intensity

C

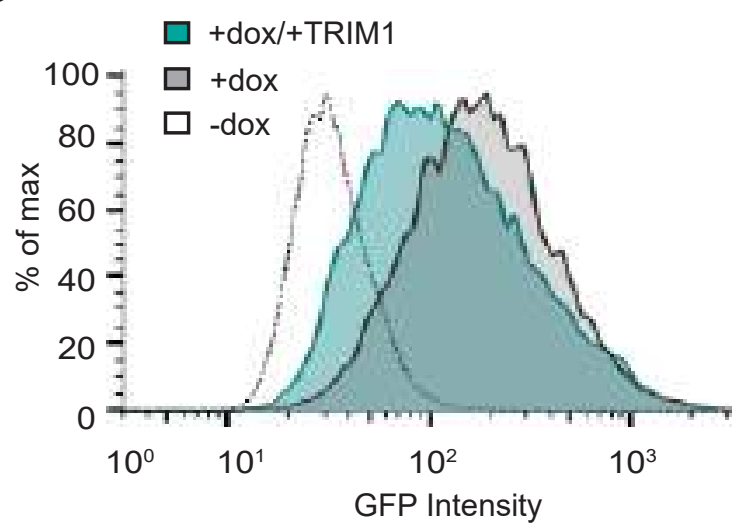

e
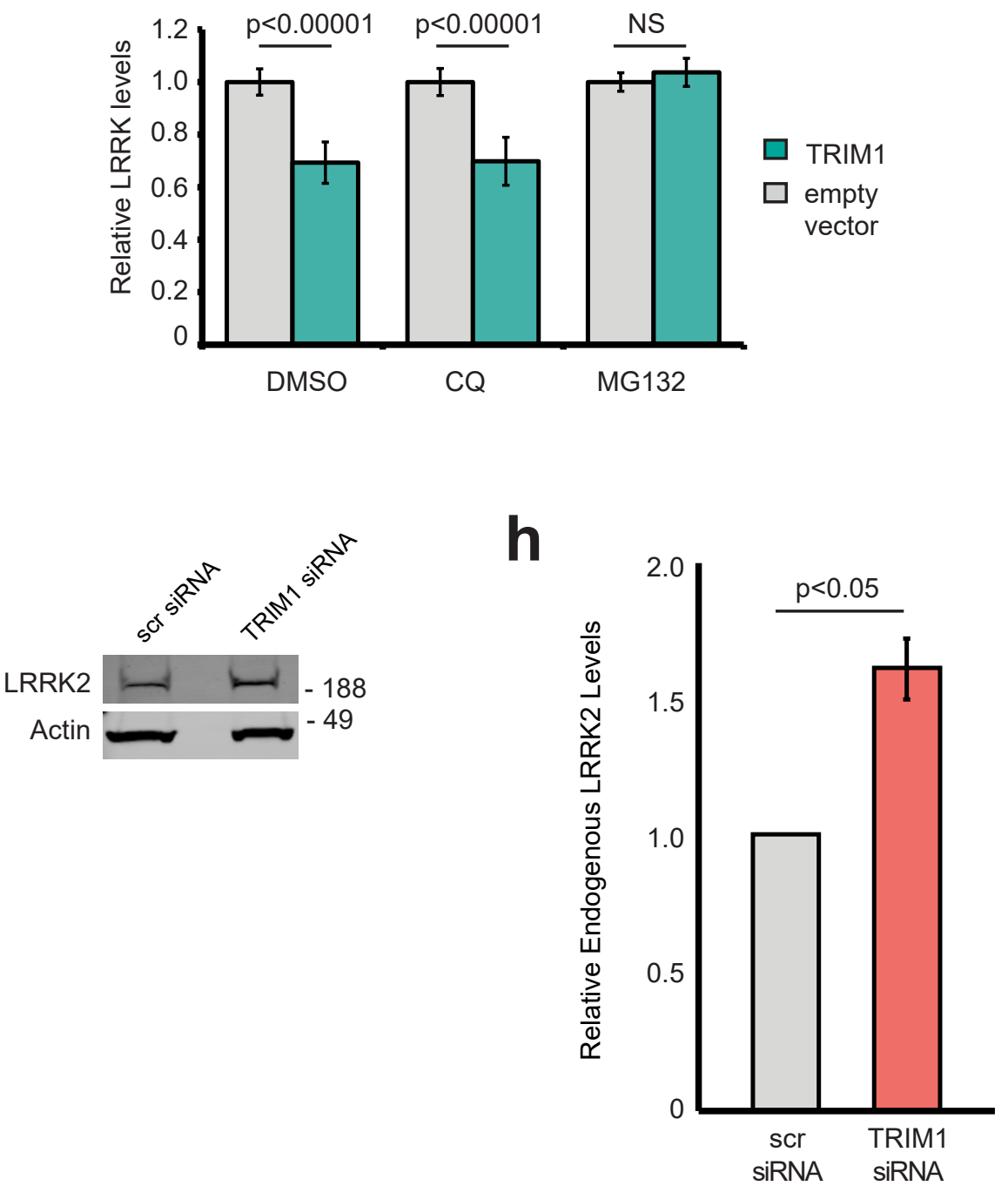
Figure 4

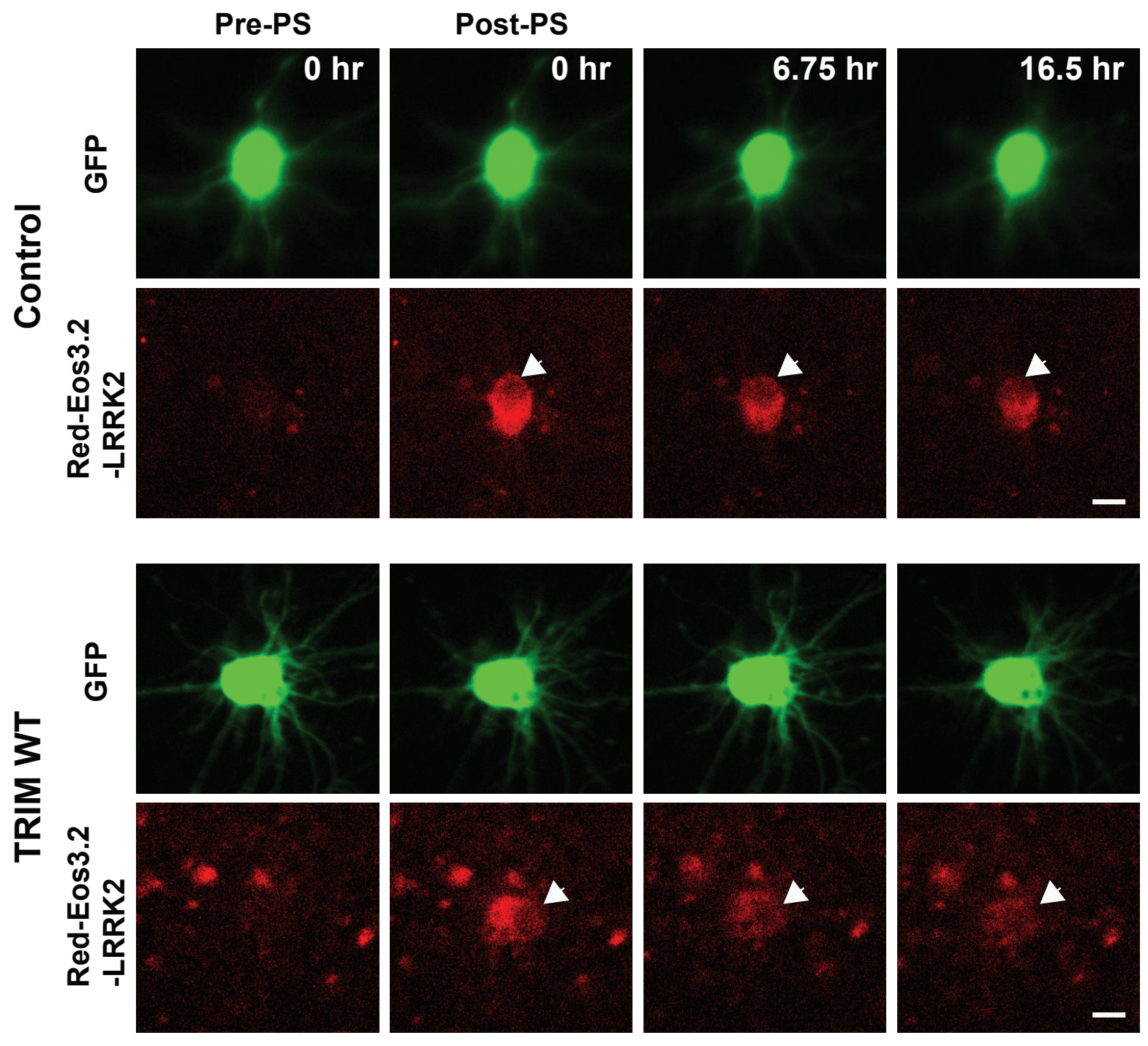


FigpiqT Fieprint doi: https://doi.org/10.1101/2020.10.21.336578; this version posted October 21, 2020. The copyright holder for this preprint (which was not certified by peer review) is the author/funder. All rights reserved. No reuse allowed without permission.

a

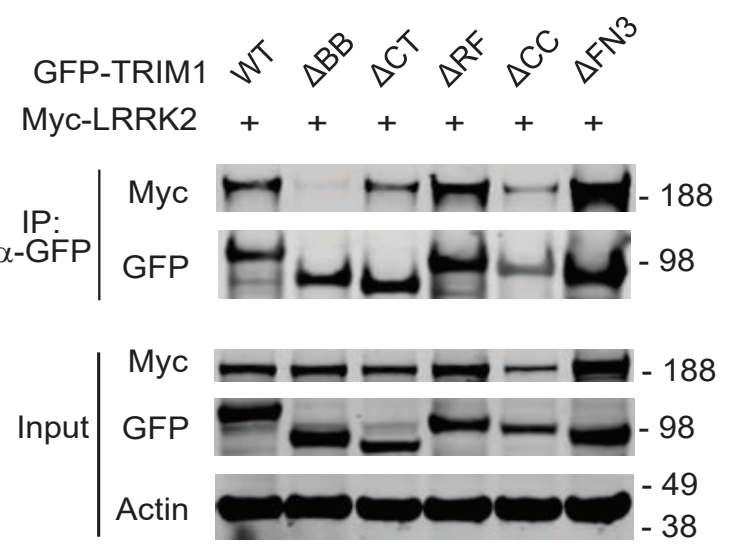

b
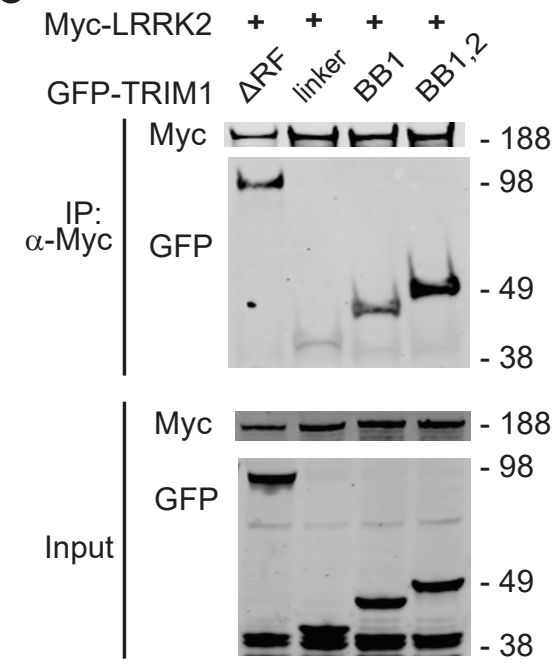

e

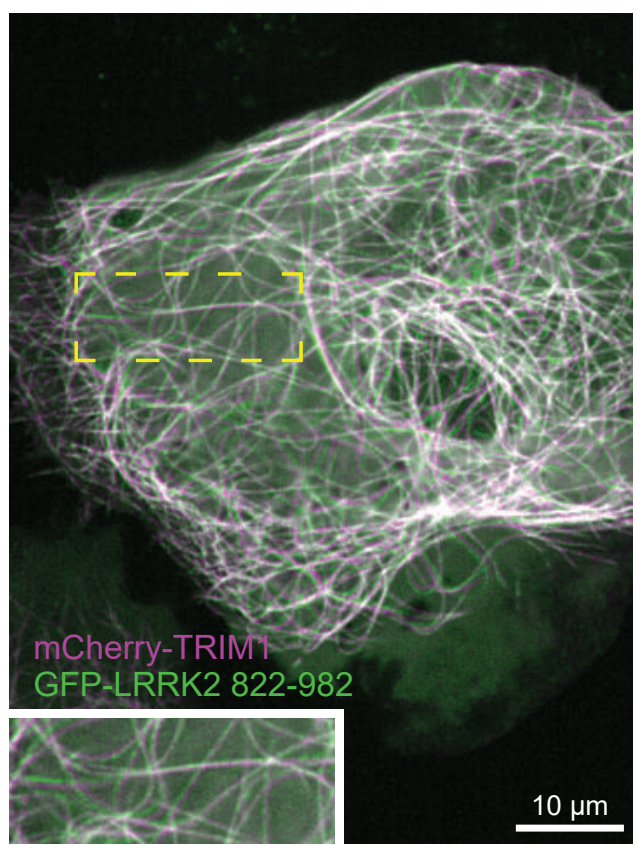

C

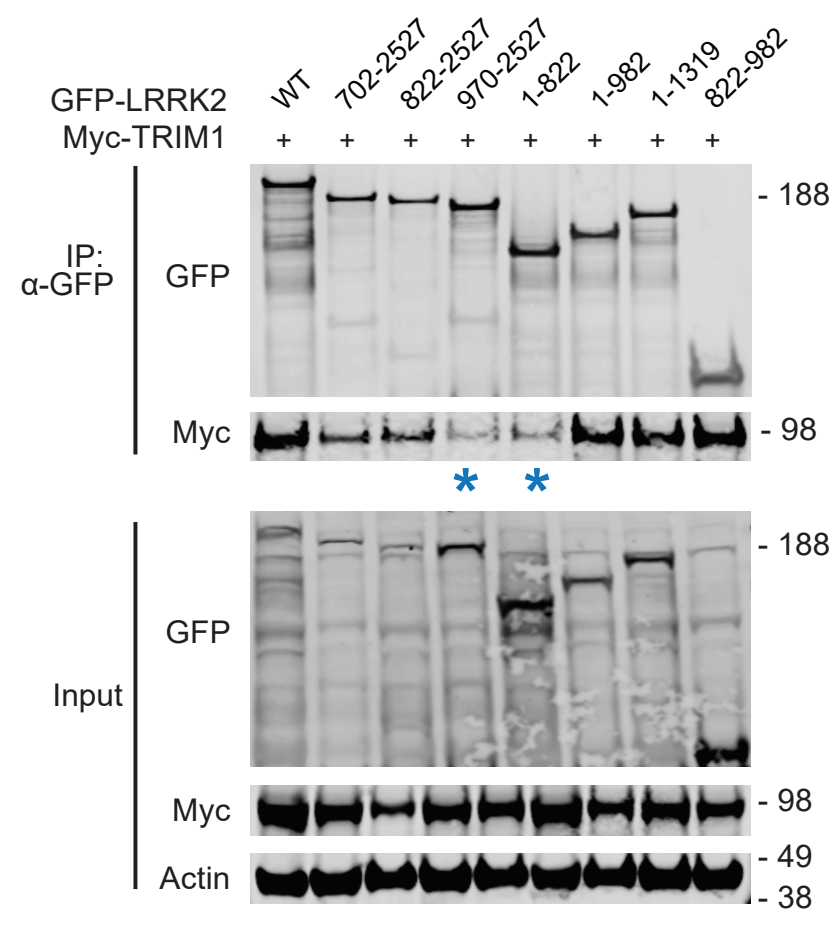

d

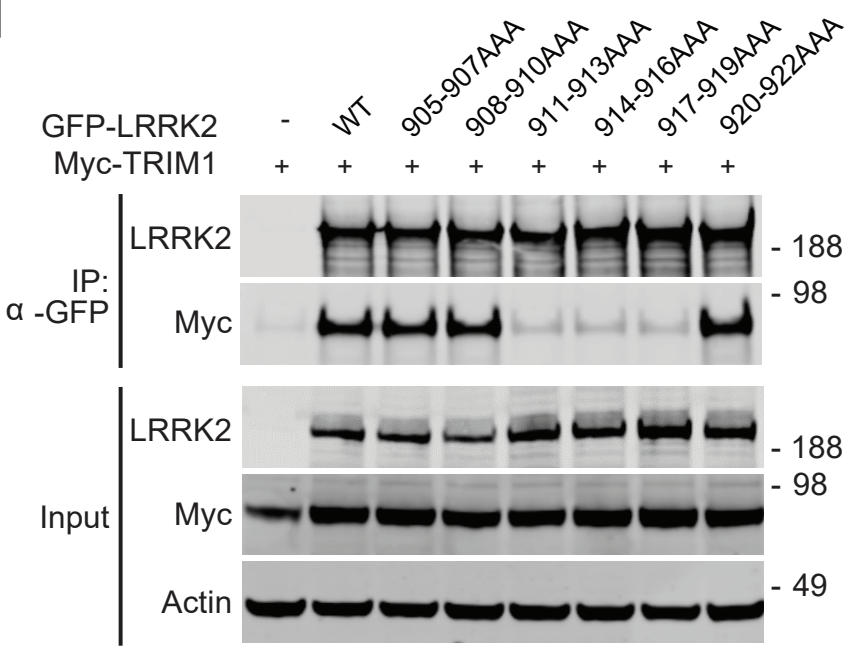

f

\section{RL}

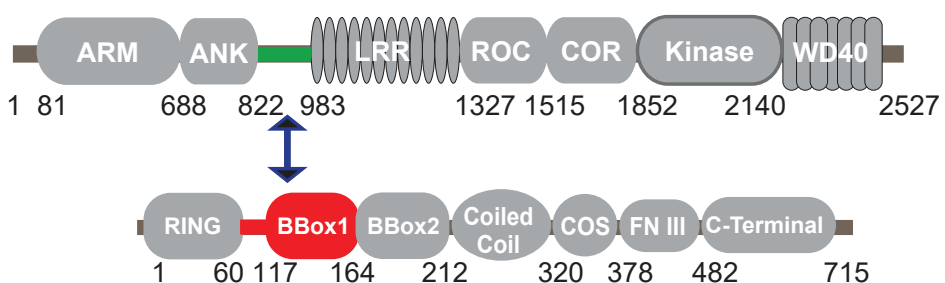




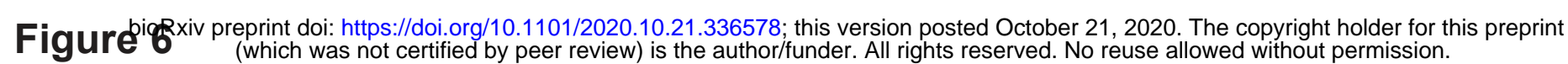
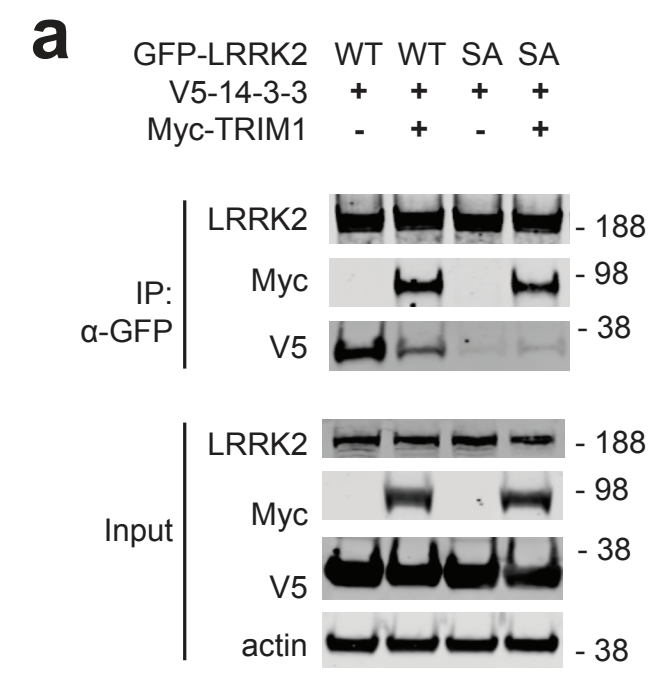

b

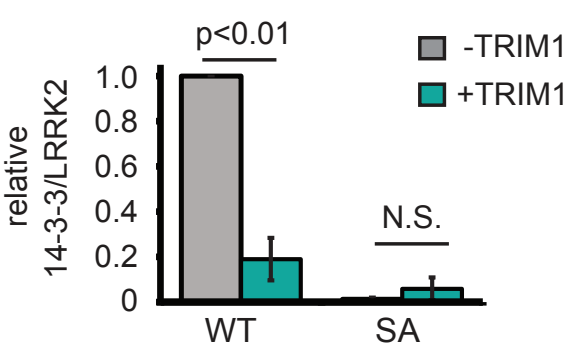

C

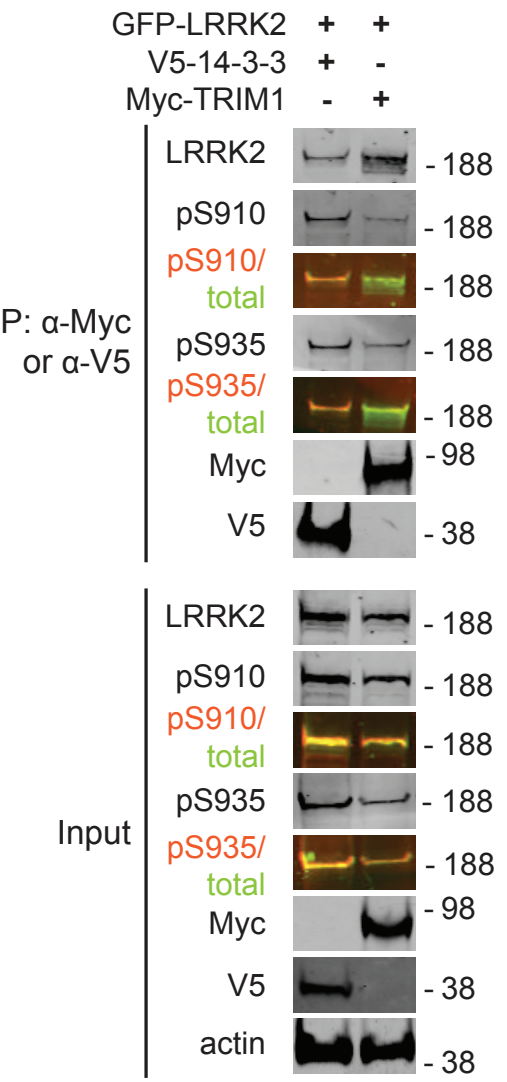

d
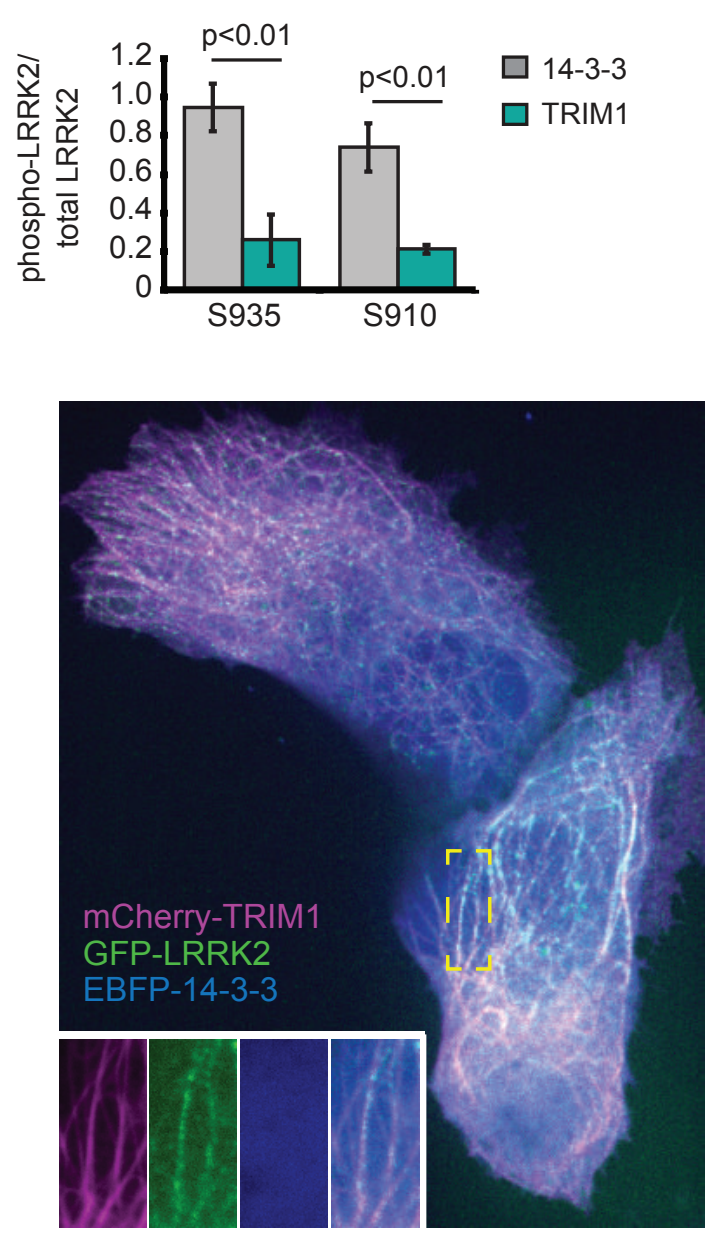


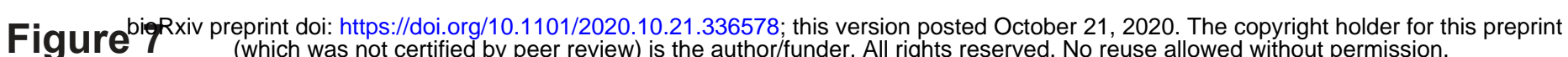
(which was not certified by peer review) is the author/funder. All rights reserved. No reuse allowed without permission.

a

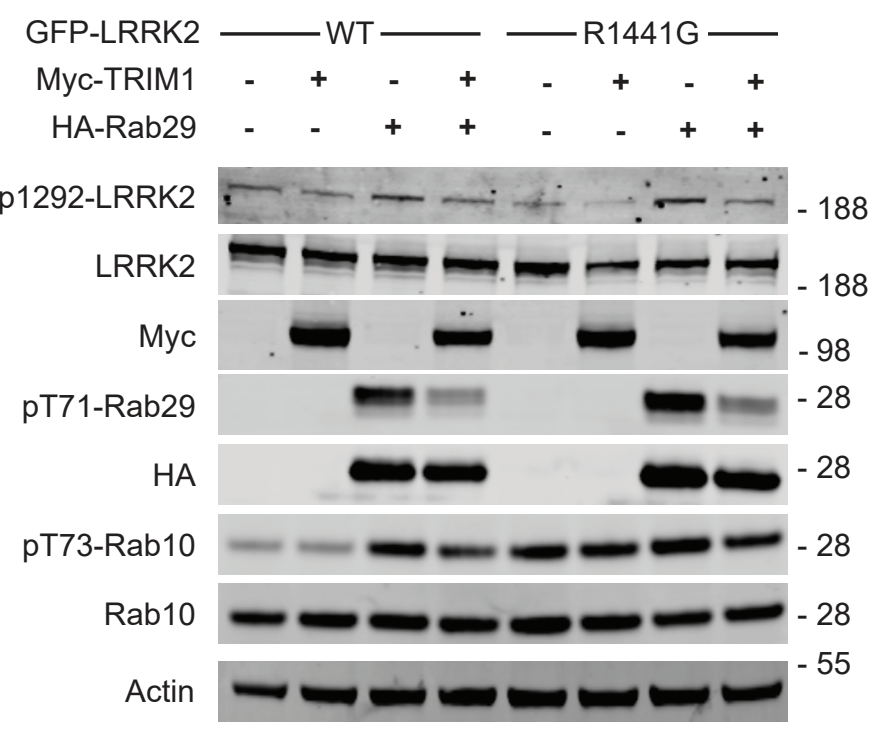

b

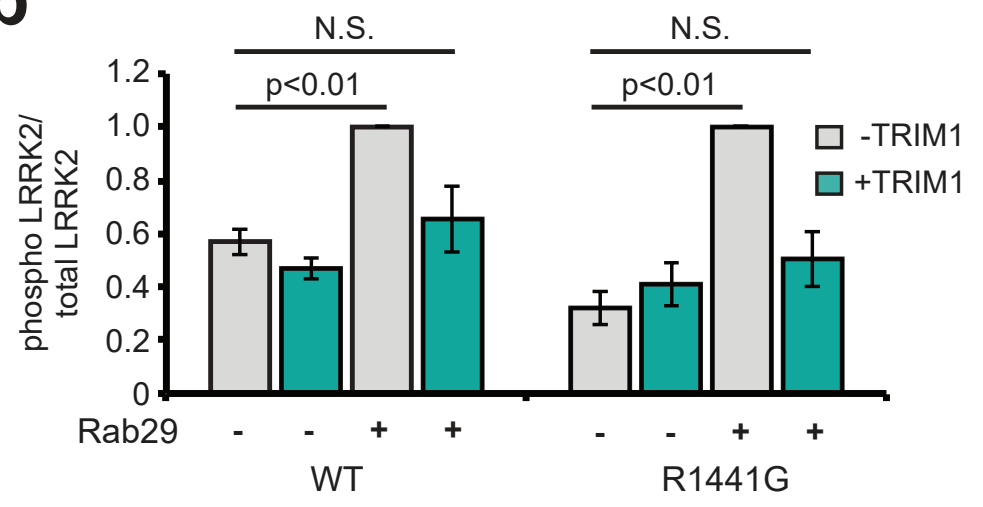

C

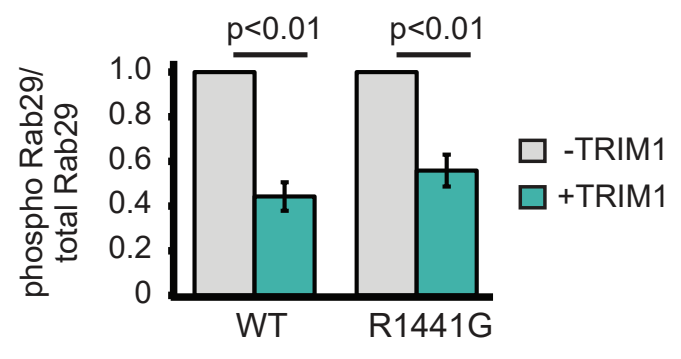

d

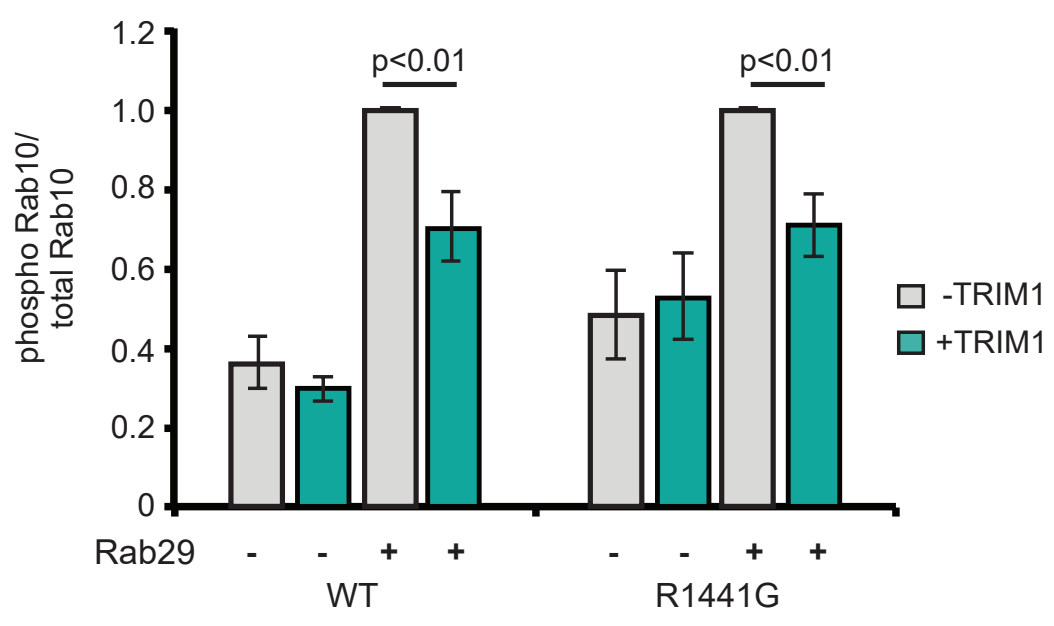

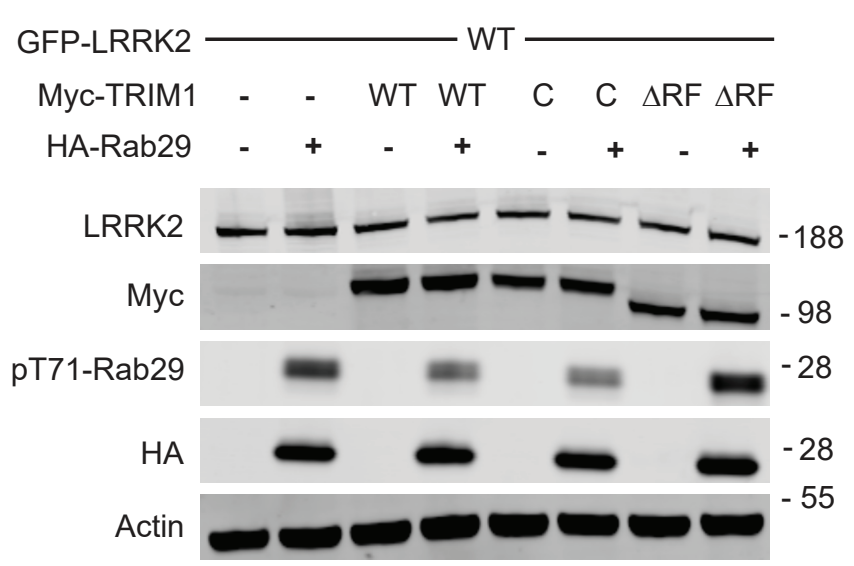

f

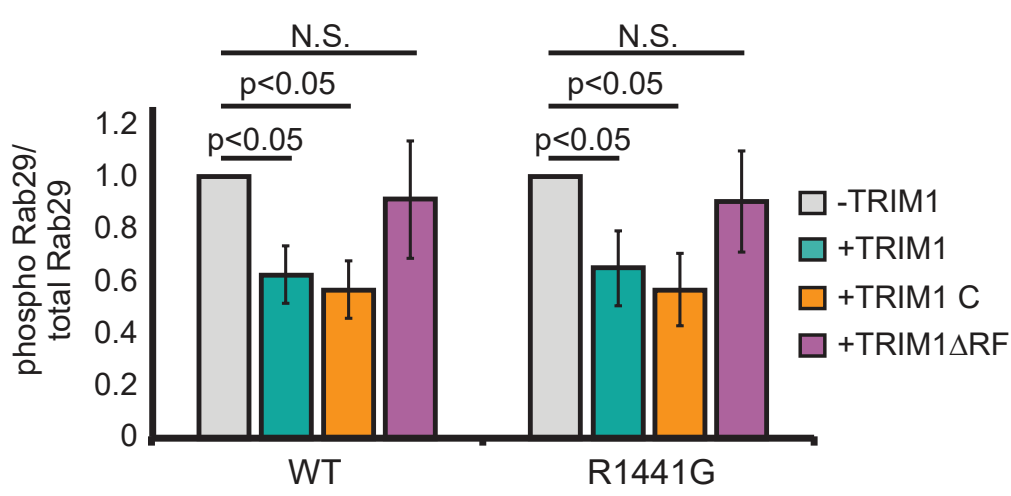

g

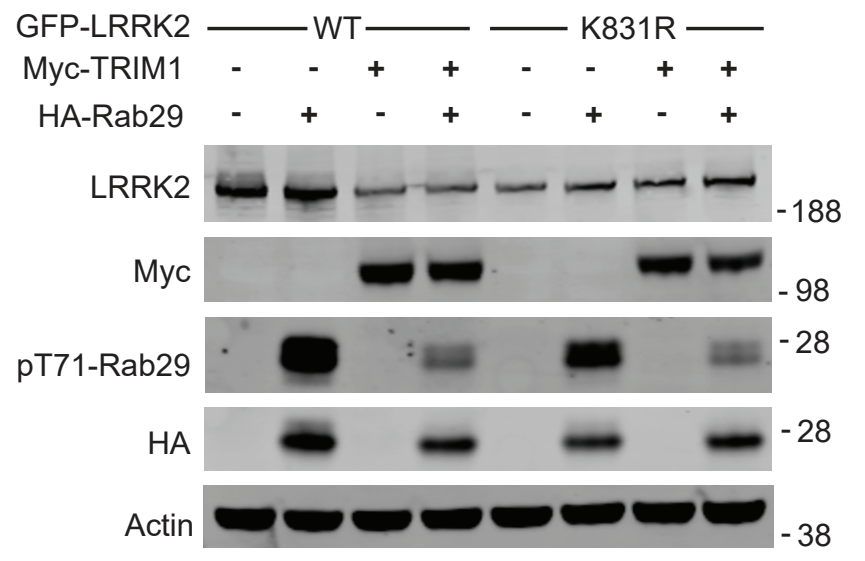

h

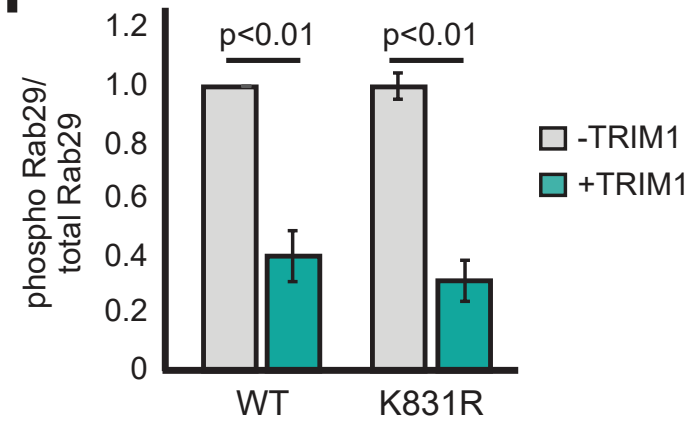



Fig eiqPexingreprint doi: https://doi.org/10.1101/2020.10.21.336578; this version posted October 21, 2020. The copyright holder for this preprint
(which was not certified by peer review) is the authorfunder. All rights reserved. No reuse allowed without permission.

a
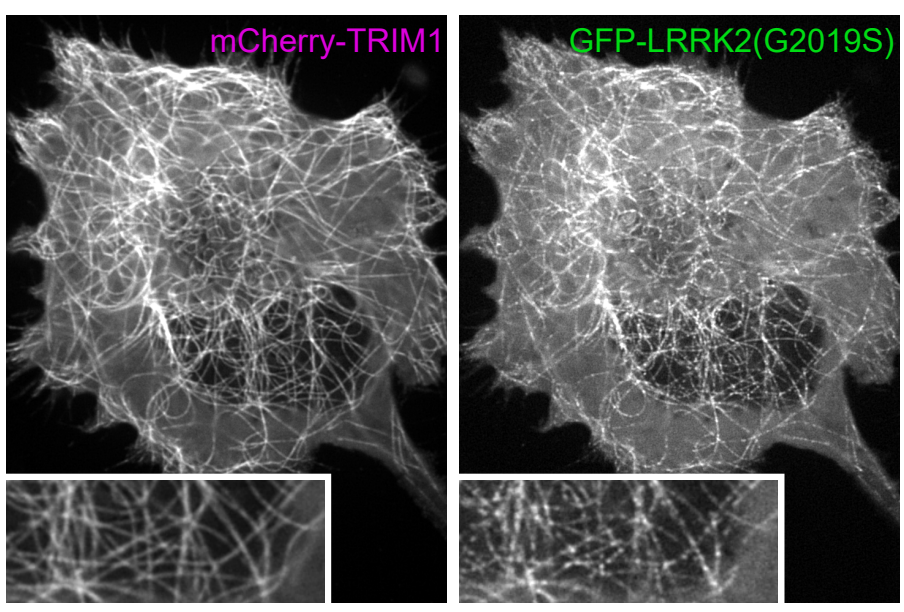

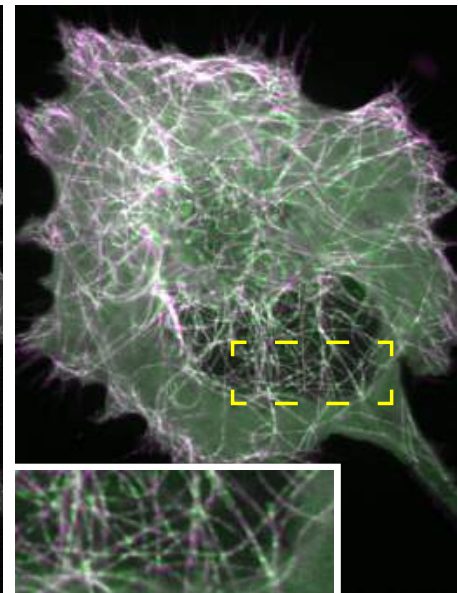

C

TRIM1

$\square$ empty vector

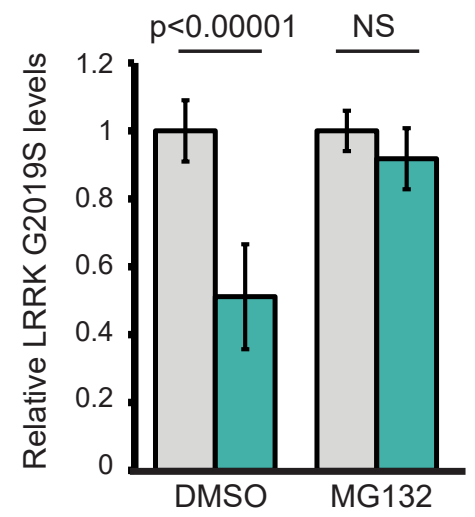

b

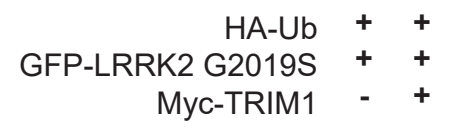

\begin{tabular}{r|llll} 
IP: & Myc & HAP & -188 \\
a-FLAG & HA & &
\end{tabular}

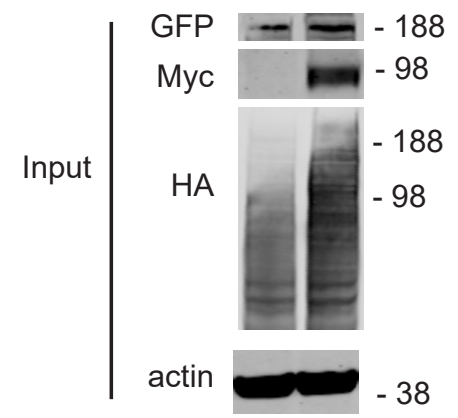

e

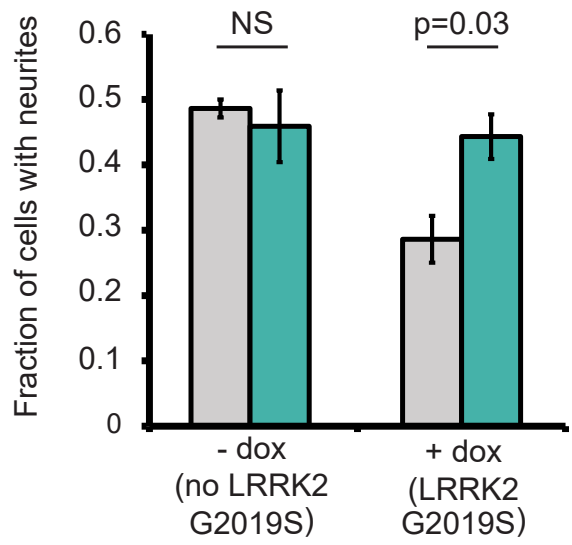

d
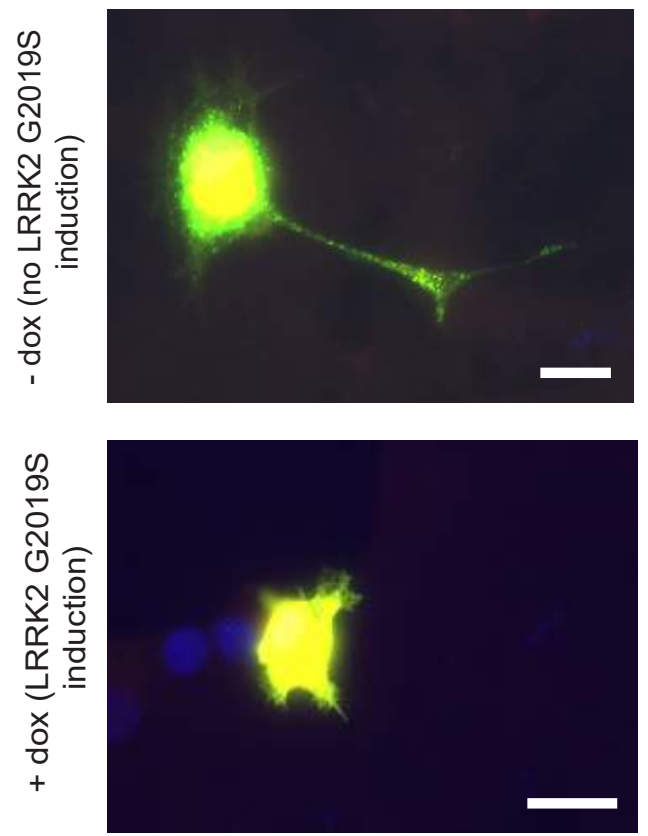

TRIM1
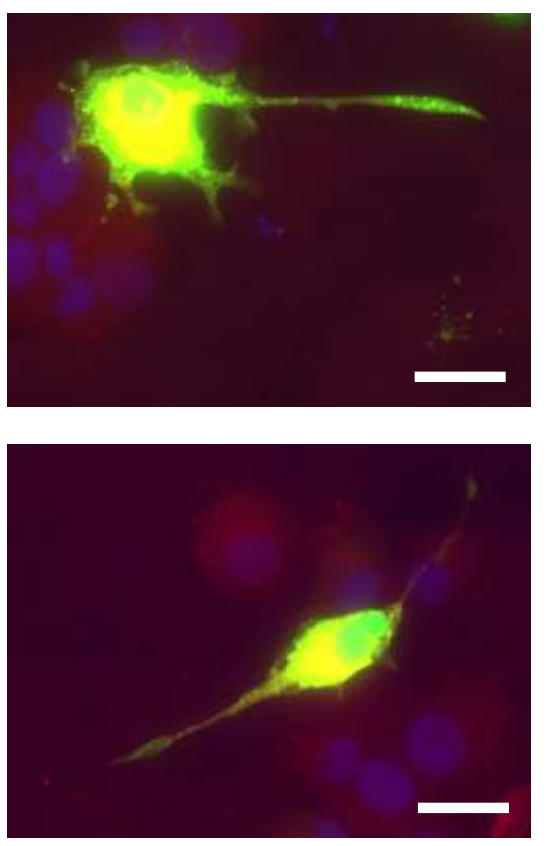

- TRIM1

$\square$ empty

vector

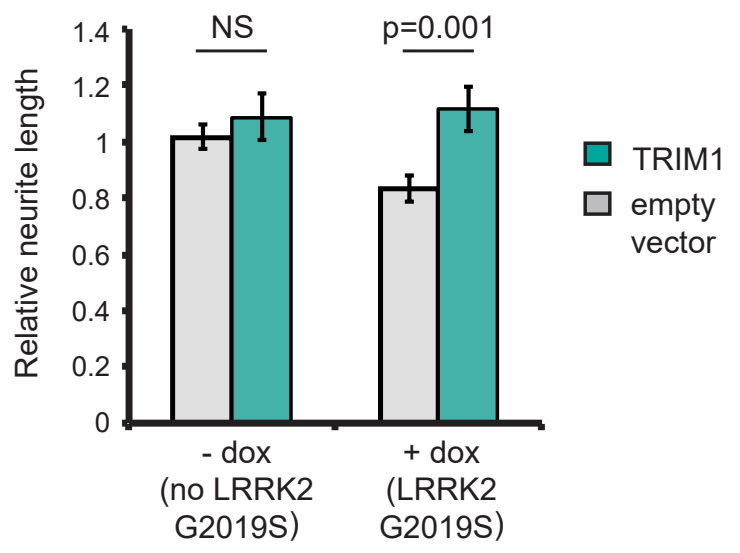


bioRxiv preprint doi: https://doi.org/10.1101/2020.10.21.336578; this version posted October 21, 2020. The copyright holder for this preprint

Figure 9 (which was not certified by peer review) is the author/funder. All rights reserved. No reuse allowed without permission.

\section{Cytoplasm}

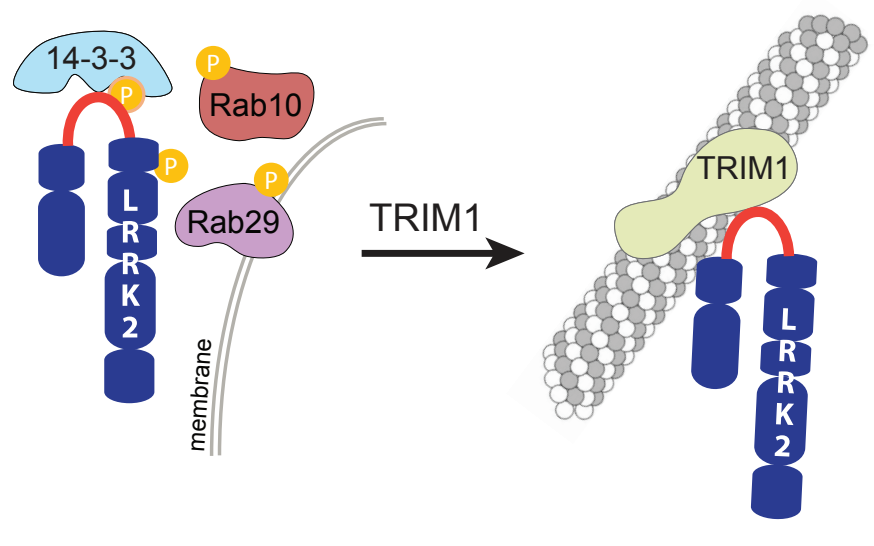

Microtubule

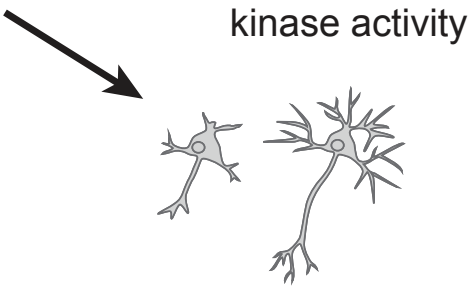

neurite outgrowth 
bioRxiv preprint doi: https;//doi.org/10.1101/2020.10.21.336578; this version posted October 21, 2020. The copyright holder for this preprint

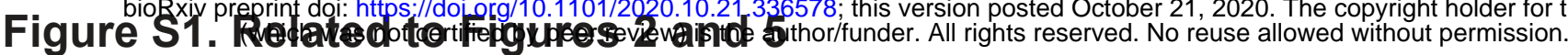

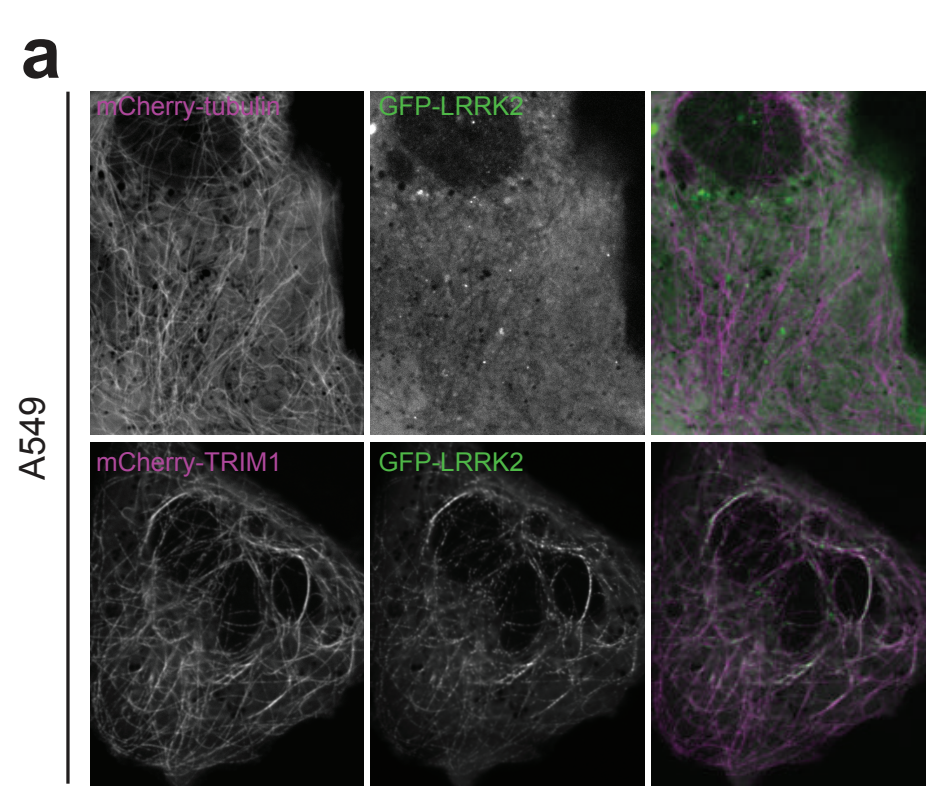

0 *

TRIM1

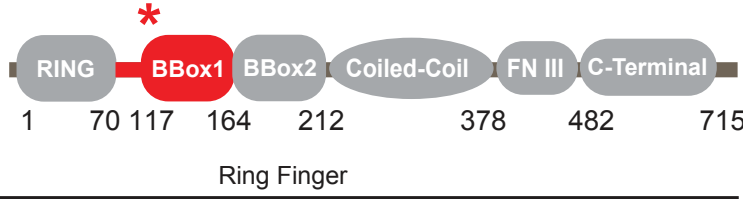

TRIM1

TRIM1

METLESELTCPICLELFEDPLLLPCAHSLCFN

METLESELTCPICLELFEDPLLLPCAHSLCE

AHRILVS

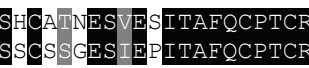

linker and B-box1

TRIM18 61 HVIIISQRGLDGLKRNVTLQNIIDRFQKASVSGPNSPSETRRERAFDANTMTSAEKVLCQ TRIM

B-box2

$\begin{array}{llll}\text { TRIM18 } & 121 & \text { FCDQDPAODAVKTCVTCEVSYCDECLKATHPNKKPFTGHRLIEPIPDSHIRGIMCLEHED } \\ \text { TRIM1 } & 121 & \text { FCEQDPPRDAVKTCITCEVSYCDRCLRATHPNKKPFTSHRLVEPVPTHLRGITCLDHEN }\end{array}$

Coiled coil

TRIM18 181 EKVNMYCVTDDQLICALCKLVGRHRDHQVAAISERYDKLKQNLESNLTNLIKRNTELETI

TRIM1 181 EKVNMYCVSDDQLICALCKLVGRHRDHQVASTNDRFEKLKQTLEMNLTNLVKRNSELENQ

Coiled coil

TRIM18 241 LAKLIQTCQHVEVNASRQEAKLTEECDLLIEI IQQRRQIIGTKIKEGKVMRLRKLAQQIA TRIM1 241 MAKLIQICOQVEVNTAMHEAKLMEECDELVEIIQQRKQMIAVKIKETKVMKLRKLAQQVA

Coiled coil

TRIM18 301 NCKQCIERSASLISQAEHSLKENDHARELQTAKNITERVS MATASSQVLIPEINLNDTFE

TRIM1 301 NCRQCIERSTVLINQAEHILKENDQARFLQSAKNIAERVAMATASSQVLIPDINFNDAEE Fibronectin III

TRIM18 361 TFALDFSREKKLLECLDYLTAPNPPIIREELCTASYDTITVHWTSDDEFSVVSYELQYTI TRIM1 361 NEALDFSREKKLLEGLDYLTAPNPPSIREELCTASHDTITVHWISDDEFSISSYELQYTI AAA

Fibronectin III

TRIM18 421 FTGQANVVSICNSADSWMIVPNIKQNHYTVHGLQSGTKYIFMVKAINQAGSRSSEPGKLK $\begin{array}{lll}\text { TRIM1 } & 421 \text { FTGQANFISIYNSVDSWMIVPNIKQNHYTVHGLQSGTRYIFIVKAINQAGSRNSEPTRLK }\end{array}$

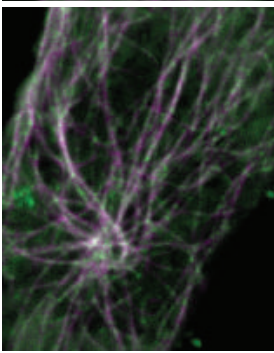

C-terminal domain (SPRY)

$\begin{array}{llll}\text { TRIM18 } & 481 & \text { TNSQPFKLDPKSAHRKLKV SHDNLTVERDESSSKKSHTPERFTSQGS YGVAGNVFIDSGR } \\ \text { TRIM1 } & 481 & \text { TNSQPFKLDPKMTHKKLKI SNDGLMEKDESSLKKSHTPERESGTGCYGAAGNIFIDSGC }\end{array}$

C-terminal domain (SPRY)

$\begin{array}{lll}\text { TRIM18 } & 541 \text { HYWEVVISGSTWYAIG AYKSAPKHEWIGKNSA SWALCRCNNNWVVRHNSKEIPIEPAPH } \\ \text { TRIM1 } & 541 \text { HYWEVVMGSSTWYAIGIAYKSAPKNEWIGKNASSWVFSRCNSN SVVRHNNKEMIVDVPPH }\end{array}$

\section{C-terminal domain (SPRY)}

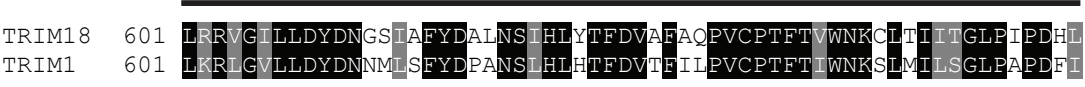

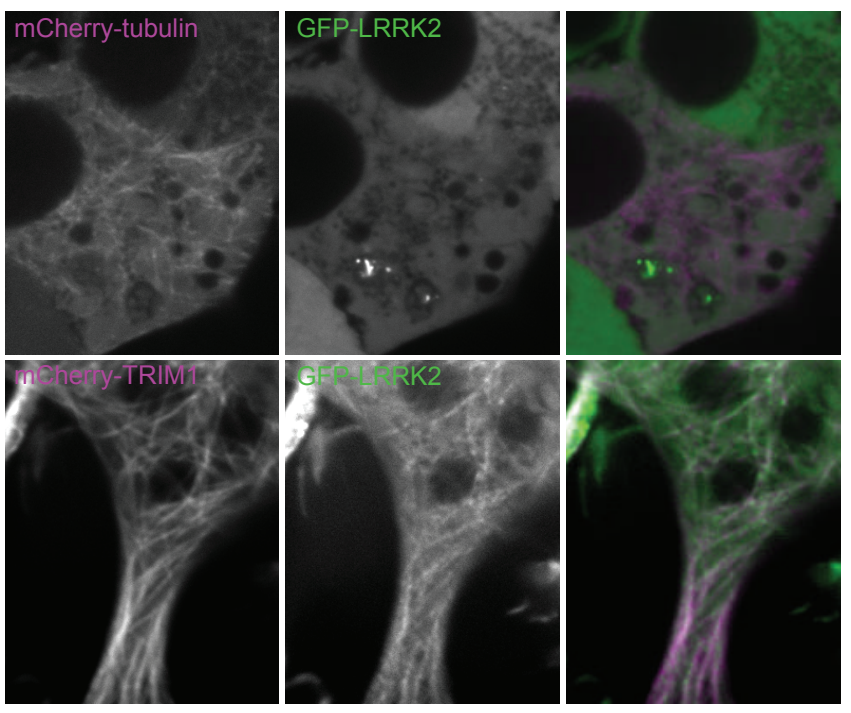

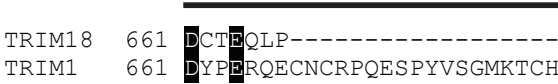

?

$$
\begin{aligned}
& \text { GFP-LRRK2 + + } \\
& \text { Myc-TRIM18 + - } \\
& \text { Myc-TRIM1 - + } \\
& \begin{array}{r|r}
\text { IP: } & \text { LRRK2 } \\
\text { a-GFP } & -98
\end{array} \\
& \begin{array}{l|l}
\text { Input } & -188 \\
& \text { LRRK2 } \\
\text { Myc } & -98 \\
\text { Actin } &
\end{array}
\end{aligned}
$$

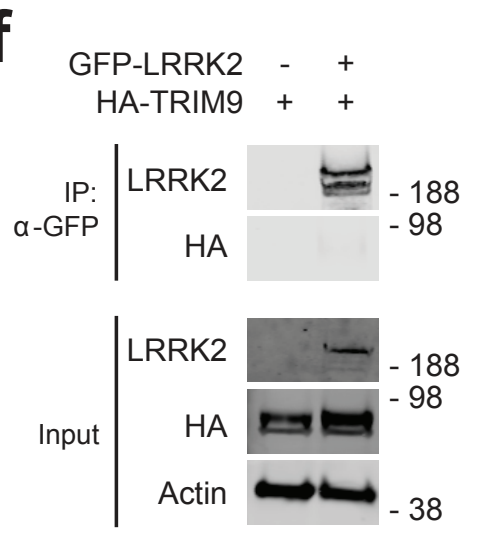


bioRxiv preprint doi: https://doi.org/10.1101/2020.10.21.336578; this version posted October 21, 2020. The copyright holder for this preprint (which was not certified by peer review) is the author/funder. All rights reserved. No reuse allowed without permission.

\section{Figure S2. Related to Figure 3.}

a

$\begin{array}{rcccc}\text { HA-Ub } & + & + & + & + \\ \text { Flag-LRRK2 } & + & + & + & + \\ \text { Myc-TRIM18 } & - & \text { WT } & - & - \\ \text { Myc-TRIM1 } & \text { WT } & - & \Delta R F & -\end{array}$
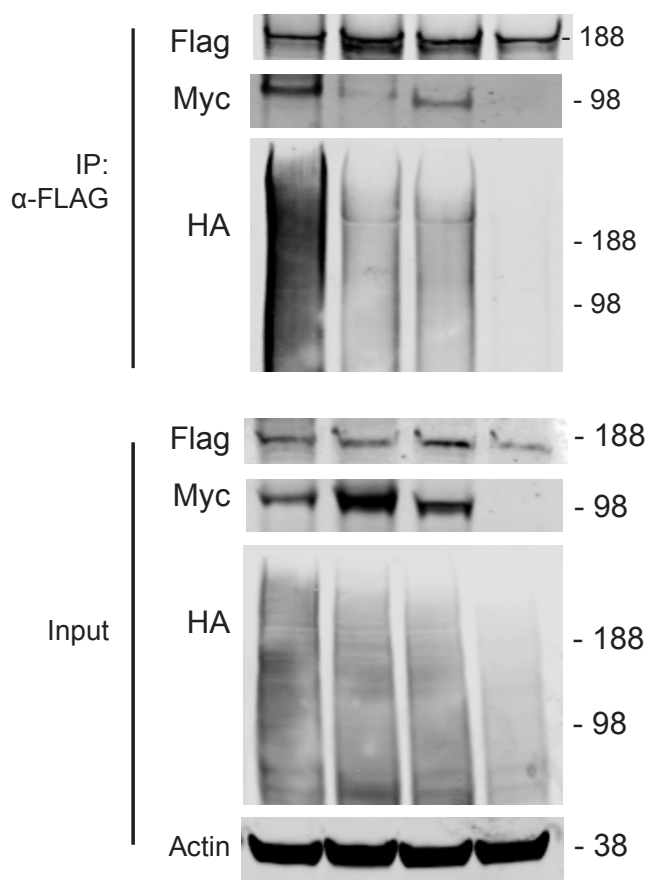

d

Hours post dox withdrawl

LRRK2

Actin

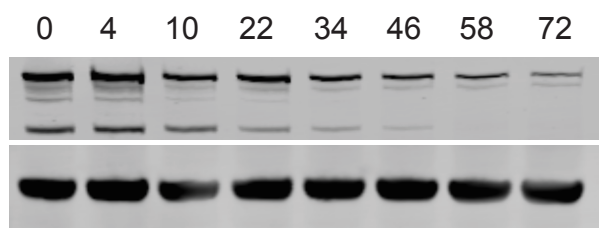

$-188$

$-38$ b

Empty vector

TRIM1

$\begin{array}{rcccccccccccccc}\text { Hours } & 2 & 6 & 10 & 16 & 24 & 32 & 48 & 2 & 6 & 10 & 16 & 24 & 32 & 48 \\ \text { FLAG-LRRK2 } & + & + & + & + & + & + & + & + & + & + & + & + & + & + \\ \text { Myc-TRIM1 } & - & - & - & - & - & - & - & + & + & + & + & + & + & +\end{array}$

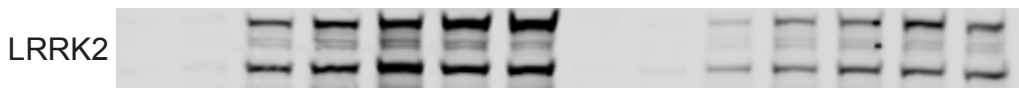

Myc

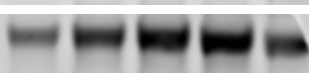

$-98$

Actin

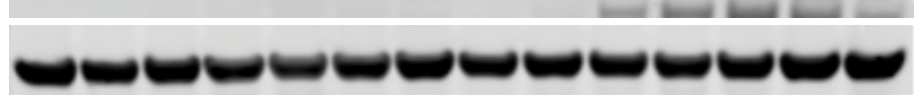

$-38$

C

LRRK2+empty vector

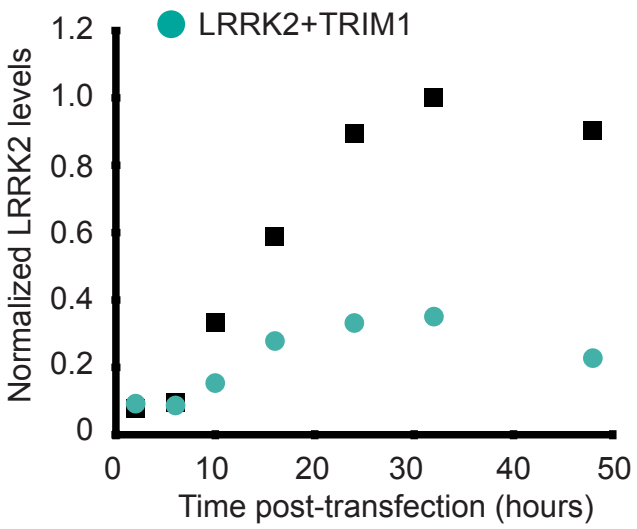

e

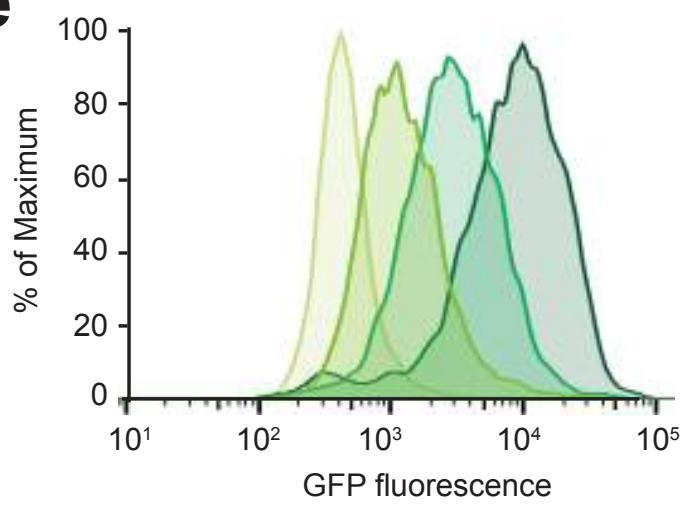

Hours post dox withdrawl:

$\square$ No Dox

$\square 0$ hours

22 hours

46 hours

f

Empty vector

TRIM1

$\begin{array}{ccccccccccccc}\text { GFP } & + & + & + & + & + & + & + & + & + & + & + & + \\ \text { Myc-TRIM1 } & - & - & - & - & - & - & + & + & + & + & + & + \\ \text { Hours } & 0 & 10 & 22 & 34 & 46 & 58 & 0 & 10 & 22 & 34 & 46 & 58 \\ \text { Post-Dox } & & & & & & & & & & & & \end{array}$

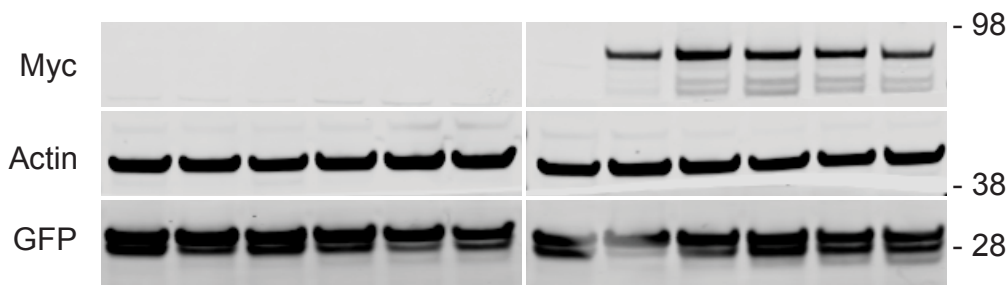


Figure S3. Related to Figure 3.
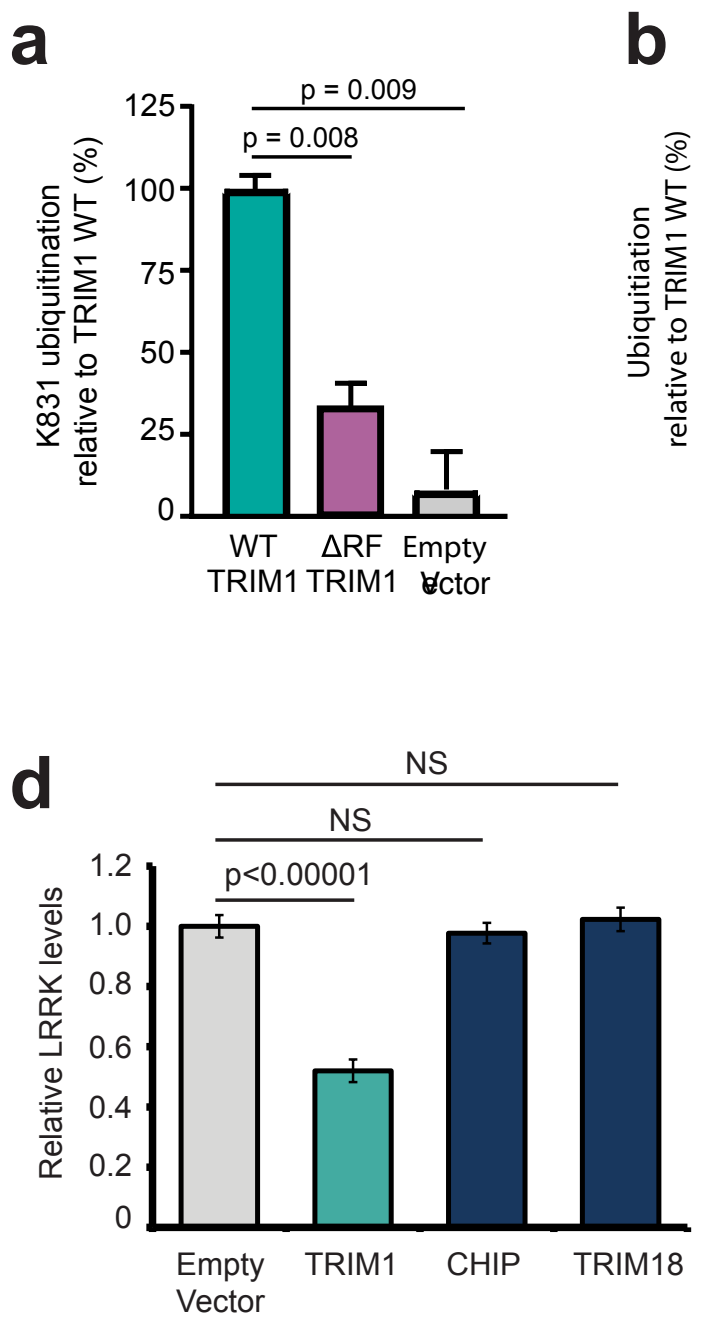

e

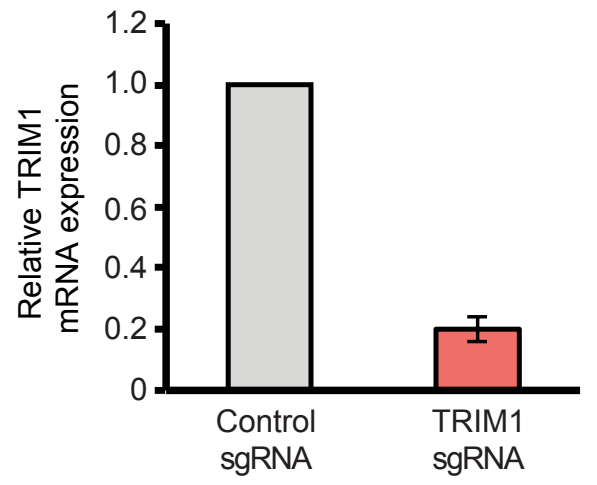

h

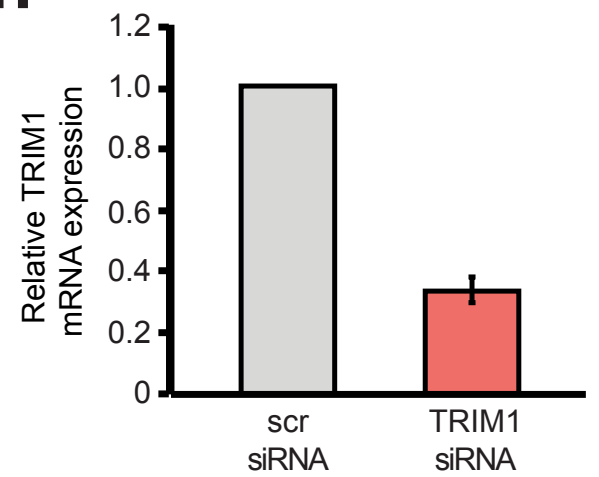

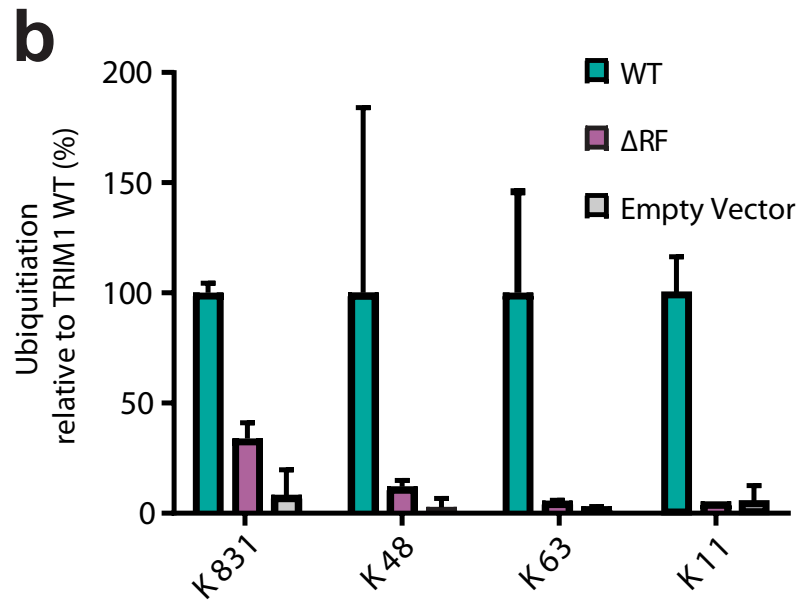

Analyte measured

$\mathbf{f}$

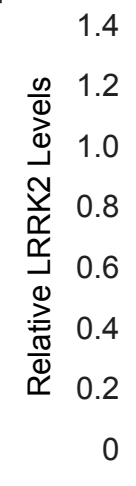

g

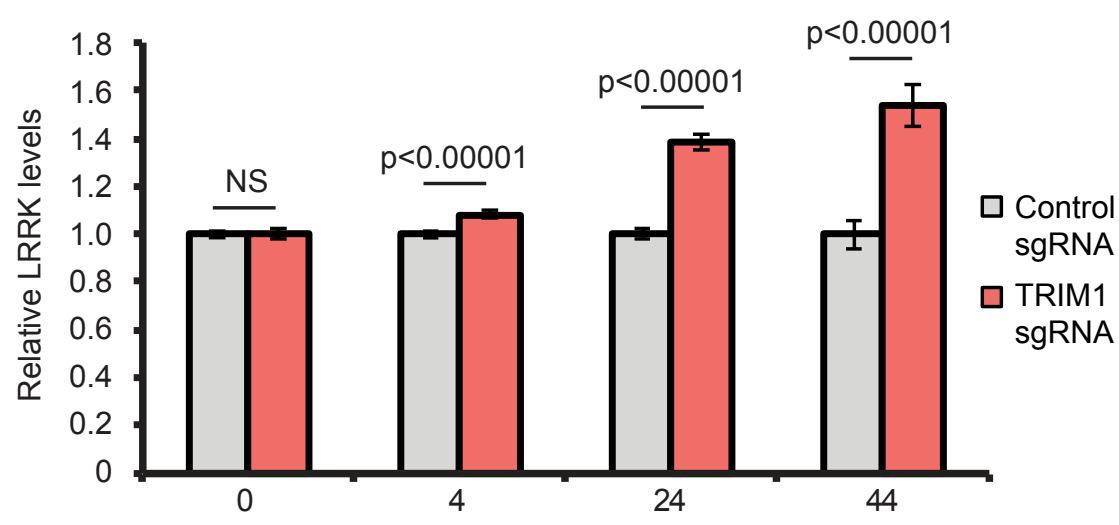

Time post-dox withdrawl (hours)

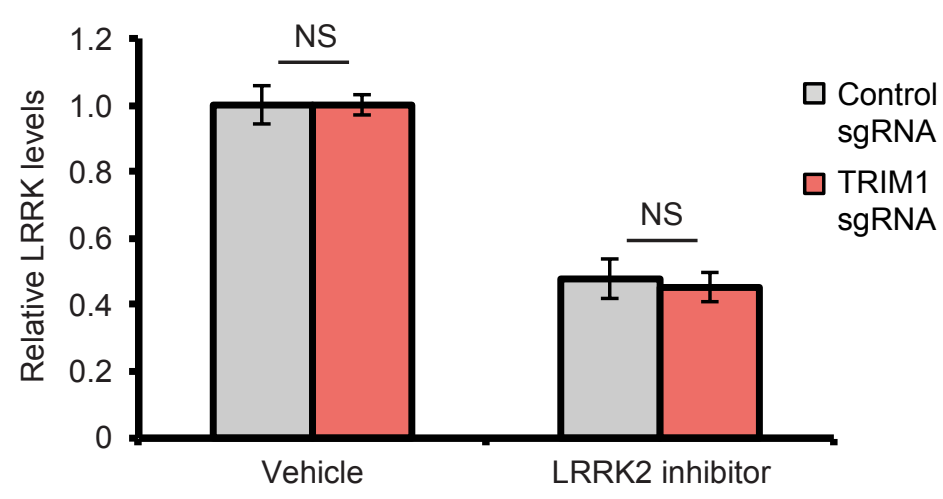


bioRxiv preprint doi: https://doi.org/10.1101/2020.10.21.336578; this version posted October 21, 2020. The copyright holder for this preprint (which was not certified by peer review) is the author/funder. All rights reserved. No reuse allowed without permission.

Figure S4. Related to Figure 5.

Panel (below) LRRK2 Construct (a.a.) Domain schematic
A
1-2527 (Full length)
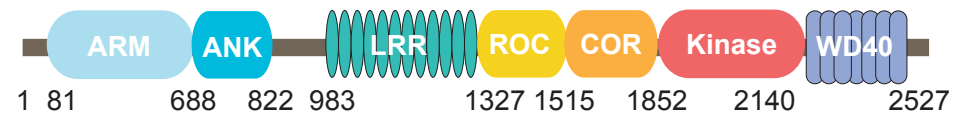

TRIM1 causes
MT localization

Co-IP

MT localization immunoblot

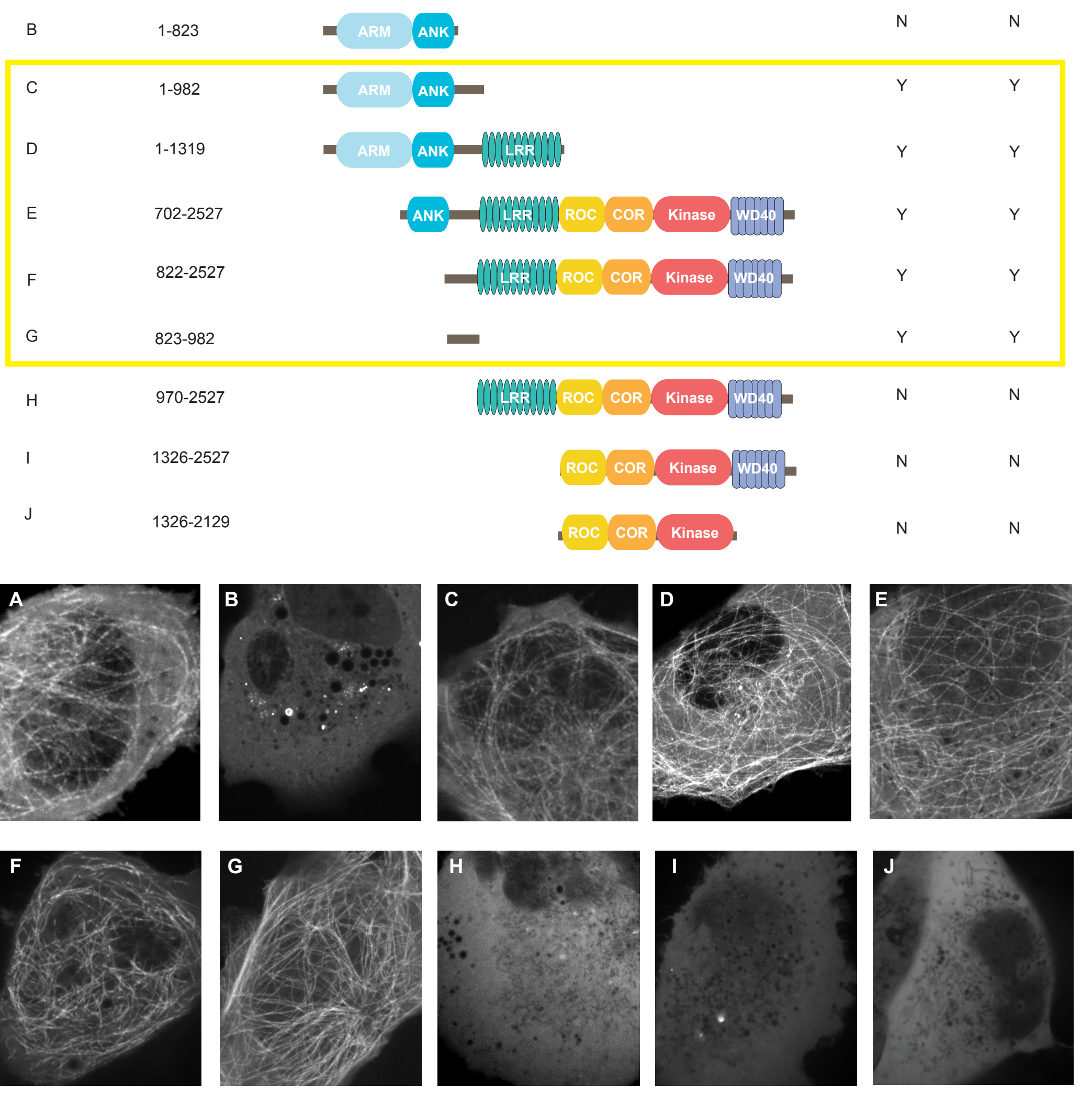




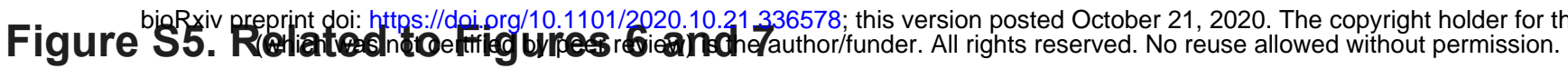

a

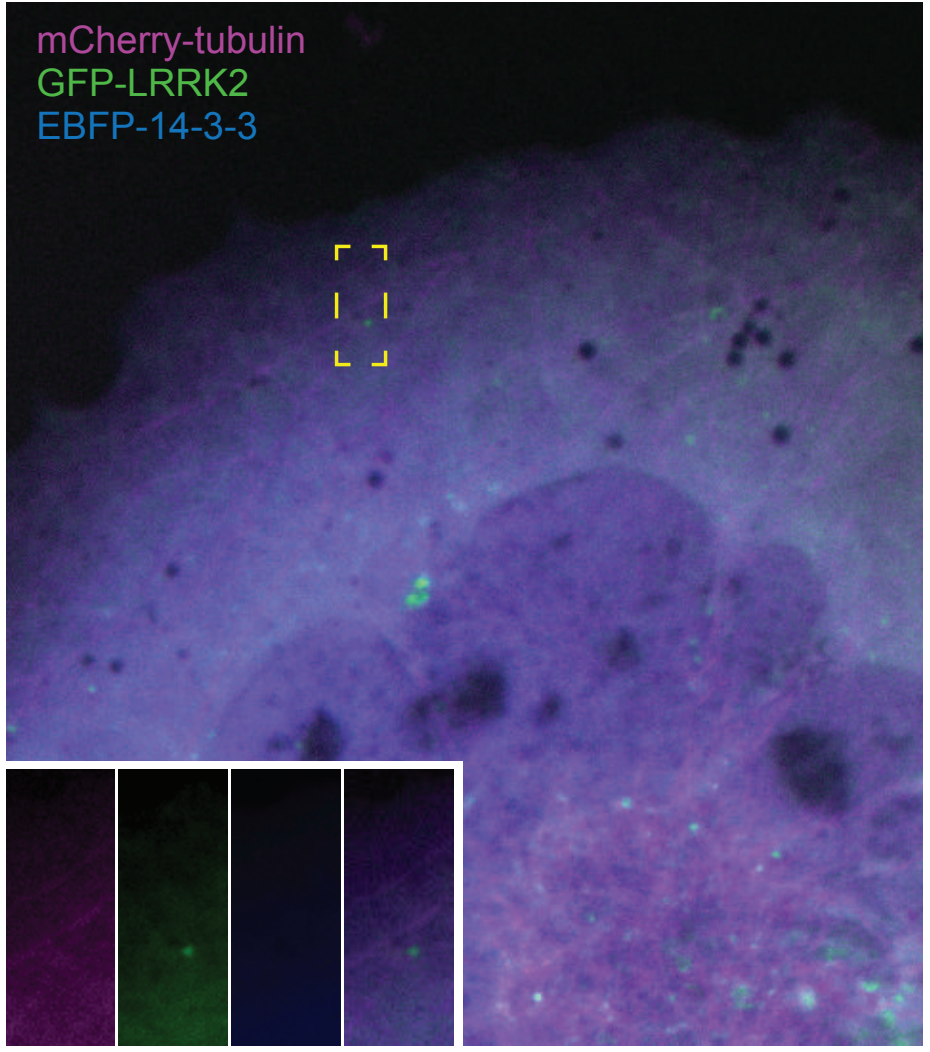

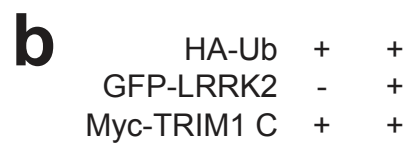
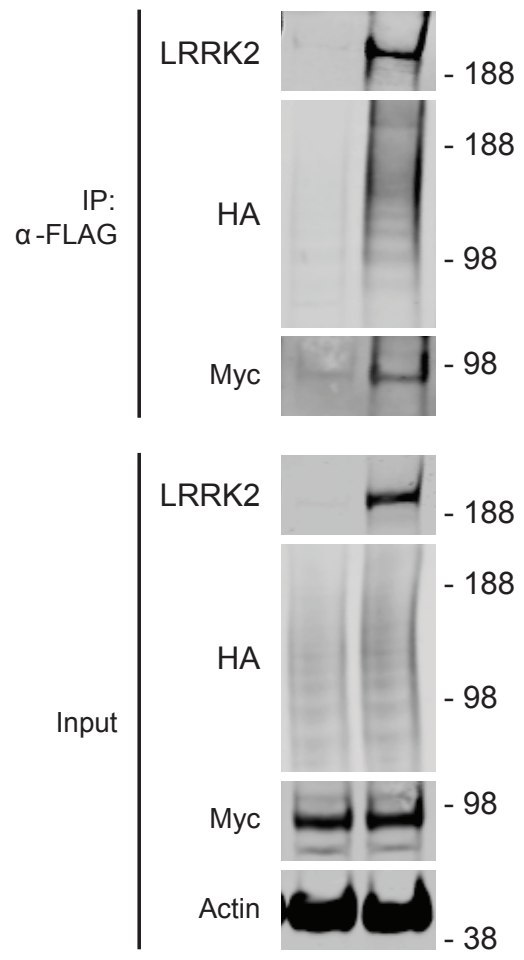

C
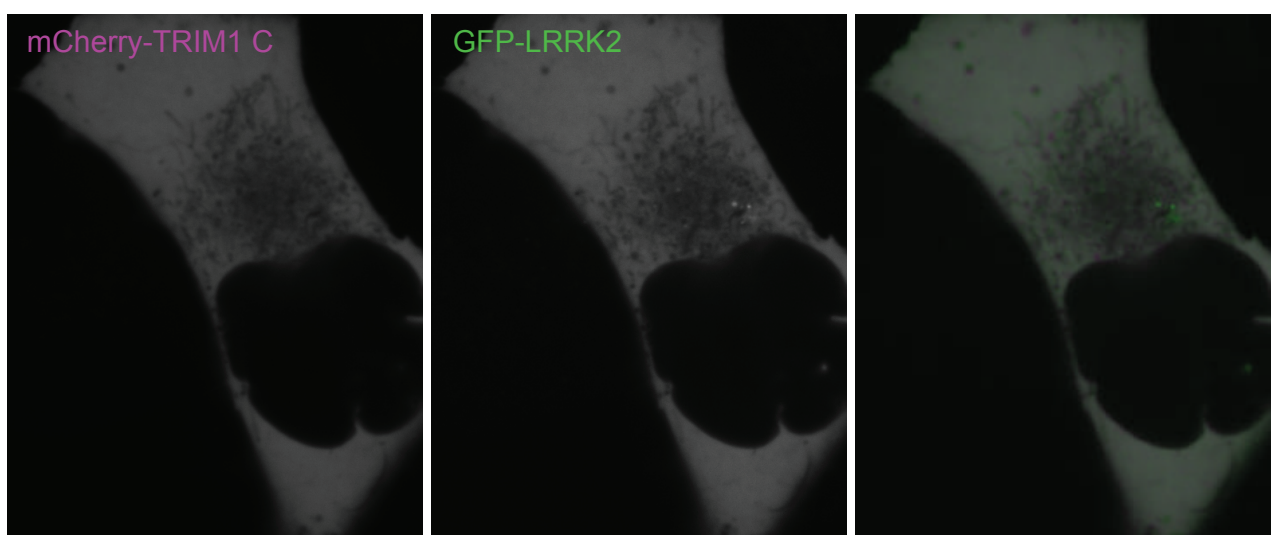

d

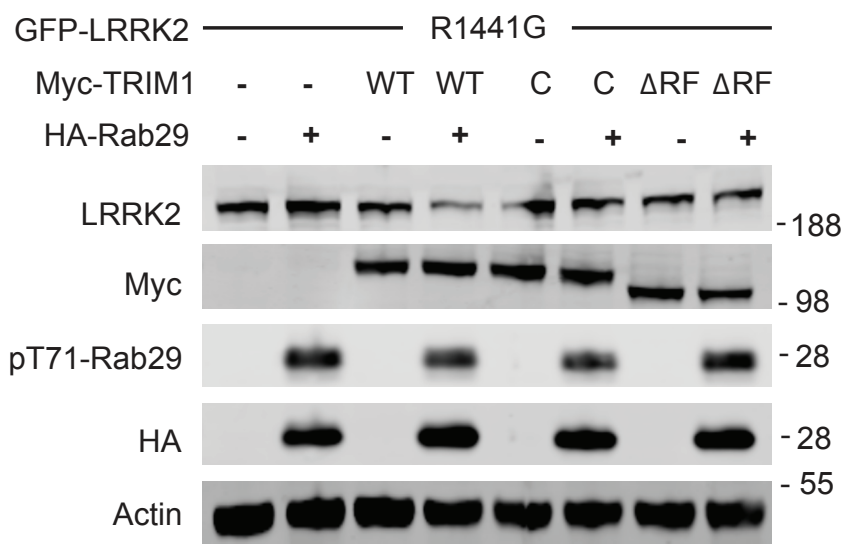

